\title{
Health technology assessment of organizational innovation in health care : the case of shared care in hearing aid provision
}

Citation for published version (APA):

Grutters, J. P. C. (2008). Health technology assessment of organizational innovation in health care : the case of shared care in hearing aid provision. [Doctoral Thesis, Maastricht University]. Universiteit Maastricht. https://doi.org/10.26481/dis.20080215jg

Document status and date:

Published: 01/01/2008

DOI:

10.26481/dis.20080215jg

Document Version:

Publisher's PDF, also known as Version of record

Please check the document version of this publication:

- A submitted manuscript is the version of the article upon submission and before peer-review. There can be important differences between the submitted version and the official published version of record.

People interested in the research are advised to contact the author for the final version of the publication, or visit the DOI to the publisher's website.

- The final author version and the galley proof are versions of the publication after peer review.

- The final published version features the final layout of the paper including the volume, issue and page numbers.

Link to publication

\footnotetext{
General rights rights.

- You may freely distribute the URL identifying the publication in the public portal. please follow below link for the End User Agreement:

www.umlib.nl/taverne-license

Take down policy

If you believe that this document breaches copyright please contact us at:

repository@maastrichtuniversity.nl

providing details and we will investigate your claim.
}

Copyright and moral rights for the publications made accessible in the public portal are retained by the authors and/or other copyright owners and it is a condition of accessing publications that users recognise and abide by the legal requirements associated with these

- Users may download and print one copy of any publication from the public portal for the purpose of private study or research.

- You may not further distribute the material or use it for any profit-making activity or commercial gain

If the publication is distributed under the terms of Article $25 \mathrm{fa}$ of the Dutch Copyright Act, indicated by the "Taverne" license above, 


\section{Health technology assessment of organizational innovation in health care}

the case of shared care in hearing aid provision

Janneke Grutters 
(C) 2008 Janneke Grutters, Maastricht

ISBN: 978-90-9022492-3

Cover design: Bianca Verhoeven \& Janneke Grutters (met dank aan Ger \& Toon Rutten) Printed by: Datawyse 


\section{Health technology assessment of organizational innovation in health care}

the case of shared care in hearing aid provision

PROEFSCHRIFT

Ter verkrijging van de graad van doctor aan de Universiteit Maastricht,

op gezag van de Rector Magnificus, Prof. Mr. G.P.M.F. Mols, volgens het besluit van het College van Decanen, in het openbaar te verdedigen op 15 februari 2008 om 14.00 uur

door

Janneke Petronella Christina Grutters 


\section{Promotor}

Prof. dr. J.J. Manni

\section{Copromotores}

Dr. L.J.C. Anteunis

Dr. M.A. Joore

Dr. F. van der Horst

\section{Beoordelingscommissie}

Prof. dr. B. Kremer (voorzitter)

Prof. dr. W.N.J. Groot

Prof. dr. J.L. Severens

Prof. dr. ir. A.F.M. Snik (Radboud Universiteit Nijmegen)

Dr. T. van der Weijden

The studies in this thesis are in part supported by grants from the Dutch Health Care Insurance Board (College voor Zorgverzekeringen, CvZ), the Heinsius Houbolt Foundation and the Dutch Hearing Foundation (Nationale Hoorstichting).

Publication and distribution of this thesis was financially supported by Stichting Atze Spoor Fonds, Progress Hearing B.V., Oticon Nederland B.V., Siemens Audiologie Techniek BV, Veenhuis Medical Audio B.V., EmiD audiologische apparatuur, Beter Horen, Beltone Netherlands BV, Streukens Hooroplossingen B.V. and Schoonenberg Hoorcomfort. 


\section{Contents}

$\begin{array}{ll}\text { Abbreviations } & 7\end{array}$

$\begin{array}{lll}\text { Chapter } 1 \quad \text { General introduction } & 11\end{array}$

$\begin{array}{lll}\text { Part I } & \text { Barriers and facilitators } & 27\end{array}$

Chapter 2 Potential barriers and facilitators for implementation of an integrated care pathway for hearing-impaired persons: an exploratory survey among patients and professionals

$\begin{array}{ll}\text { Part II } \quad \text { Outcome measurement } & 47\end{array}$

Chapter 3 Choosing between measures: comparison of EQ-5D, HUI2 and HUI3 in persons with hearing complaints

Chapter 4 Willingness to Pay for a hearing aid: comparing the payment scale and open-ended question

Chapter 5 Willingness to Accept versus Willingness to Pay in a Discrete

Choice Experiment

Chapter 6 Patient preferences for direct hearing aid provision by a private dispenser. A discrete choice experiment

Part III Integration of evidence

Chapter 7 Decision-analytic modeling to assist decision-making in organizational innovation: the case of shared care in hearing aid provision

Chapter 8 General discussion

Bibliography

Summary

Samenvatting

Co-authors \& affiliations

Dankwoord

Curriculum Vitae

Publications 

Abbreviations 



\begin{tabular}{|c|c|}
\hline \multicolumn{2}{|c|}{ Abbreviations } \\
\hline $\mathrm{AC}$ & Audiological Centre \\
\hline ANOVA & Analysis of Variance \\
\hline BEPTA & Better Ear Pure Tone Average \\
\hline CBA & Cost-Benefit Analysis \\
\hline CEAC & Cost-Effectiveness Acceptability Curve \\
\hline $\mathrm{Cl}$ & Confidence Interval \\
\hline CEA & Cost-Effectiveness Analysis \\
\hline CEN & Comité Européen de Normalisation \\
\hline CMA & Cost-Minimization Analysis \\
\hline CUA & Cost-Utility Analysis \\
\hline$d B$ & Decibel \\
\hline DCE & Discrete Choice Experiment \\
\hline ENT & Ear, Nose and Throat \\
\hline $\mathrm{EO}$ & Expert Opinion \\
\hline EQ-5D & EuroQol 5D \\
\hline EVPI & Expected Value of Perfect Information \\
\hline GP & General Practitioner \\
\hline $\mathrm{HA}$ & Hearing Aid \\
\hline $\mathrm{HC}$ & Hospital Costs \\
\hline HRQOL & Health Related Quality of Life \\
\hline HTA & Health Technology Assessment \\
\hline HUI2 & Health Utilities Index Mark II \\
\hline HUI3 & Health Utilities Index Mark III \\
\hline ICC & Intra-Class Correlation Coefficient \\
\hline ICER & Incremental cost-effectiveness ratio \\
\hline IR & Interquartile Range \\
\hline $\mathrm{kHz}$ & Kilohertz \\
\hline MTA & Medical Technology Assessment \\
\hline NA & Not Applicable \\
\hline NC & Not Calculated \\
\hline OS & Observational Study \\
\hline PC & Personal Communication \\
\hline QALY & Quality Adjusted Life Year \\
\hline SD & Standard Deviation \\
\hline SE & Standard Error \\
\hline SG & Standard Gamble \\
\hline StAr & Stichting Audicienregister \\
\hline TAIL & Technology Assessment Iterative Loop \\
\hline TTO & Time Trade-Off \\
\hline UK & United Kingdom \\
\hline US & United States \\
\hline VAS & Visual Analogue Scale \\
\hline WTA & Willingness to Accept \\
\hline WTP & Willingness to Pay \\
\hline
\end{tabular}





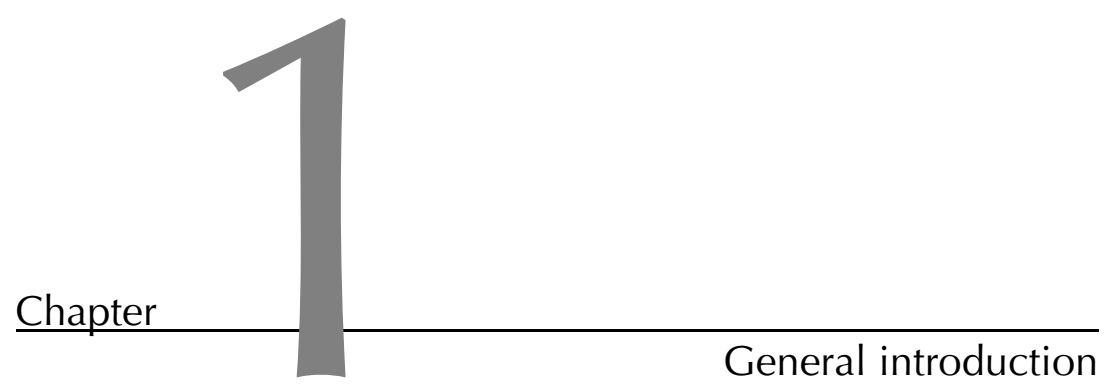



This thesis focuses on the evaluation of organizational innovation in health care. As a case, shared care in hearing aid provision is evaluated. The increasing burden of chronic diseases on today's health care system has resulted in an increasing importance of redesigning health care to patient-centered care, and of attuning the tasks of health care professionals. With regard to hearing aid provision, the current growth of the ageing population causes a proportional growth of the population with hearing impairment. This raises the burden of disease and the medical consumption for hearing impairment and, as a consequence, increases the costs of hearing rehabilitation. Increasing attention is therefore paid to attuning the tasks of all professionals involved in the care for hearing-impaired persons. This has resulted in shared care initiatives in hearing aid provision.

Health technology assessment aims to examine medical, social, ethical and economic implications of technologies in health care (Banta, 1982; Platform HTA, 2001). In this context technologies can be anything, from pharmaceuticals to organizational change. However, health technology assessment is mostly applied to medical interventions such as pharmaceuticals. Organizational innovation has rarely been the focus of health technology assessment.

The aim of the thesis is to use health technology assessment to evaluate whether an organizational innovation, shared care in hearing aid provision, benefits the care for hearing-impaired persons. This general introduction first touches upon the concepts of health and health care, to illustrate the context of the thesis. Next, the concept of organizational innovation is explained. Subsequently, it is described how health technology assessment can be used to evaluate organizational innovations. Furthermore, the case of shared care in hearing aid provision is sketched. The final part of this chapter states the general objective and research questions, and provides an outline of the thesis.

\section{Health and health care}

Health is defined by the World Health Organization as 'a state of complete physical, mental and social well-being and not merely the absence of disease or infirmity' (World Health Organization, 1946). Since its publication in 1946 this definition has been criticized and, to this very day, ongoing debates about the scope of health proceed. In these debates, consensus has emerged that health is a separate concept from well-being, that health is comprised of states or conditions of functioning of the human body and mind, and that health is an attribute of an individual person (Salomon et al., 2003).

The historical evolution of health can be described in health transition stages (Martens, 2002). Historically, health has shifted from an age of pestilence and famine, with high levels of mortality and fertility, to an age of receding pandemics. The age of receding pandemics involved a reduction of the prevalence of infectious diseases and a fall in mortality rates, high fertility rates and the introduction of modern health care and health technologies. At present we have reached the age of chronic diseases, which is characterized by improved health care, low levels of mortality and fertility, and relatively high levels of morbidity, especially for the elderly. The low levels of mortality and fertility 
lead to the ageing of the population. Hence, there is an increasing demand for health care related to the conditions of older people, where the emphasis is often more on care instead of cure.

The transition to an age of chronic illness has implications for the organization of health care (Wagner et al., 1996). More than ever patients need to be placed centre stage in the organization of health care. This is however at odds with the fact that health care professionals have grown more and more specialized, resulting in increasingly more professionals involved in the care for one condition. In such a fragmented health care system, patients are often transferred to different professionals or institutions, without complete information regarding the patient's condition, medical history and services provided in other settings. Especially in the care for older people, who often suffer from multi-morbidity, it is therefore important that health care professionals work together, and that tasks of different professionals are attuned, to allow for modernization of health care while maintaining or improving its quality.

\section{Organizational innovation in health care}

Organizational innovation in health care is a very diffuse concept, as it encompasses any change in the delivery of care. Due to the increasing attention to patient-centered care policy makers, health care providers and health insurance companies are continuously forced to seek new ways to provide the best possible care for patients within a given budget. Hence, several health delivery models have been developed to facilitate interdisciplinary care that involves the full range of health providers for a disease. Related to interdisciplinary care numerous terms such as shared care, integrated care, disease management or transmural care are used interchangeably (Grone \& Garcia-Barbero, 2001; Kodner \& Spreeuwenberg, 2002). In this thesis we define shared care as care that, geared to the needs of the patient, is provided on the basis of agreement regarding cooperation and co-ordination between health care providers, with shared responsibility and explicit sub-responsibilities (Nationale Raad voor de Volksgezondheid \& College voor Ziekenhuisvoorzieningen, 1995). The goals of shared care are to achieve effectiveness, patientorientation, continuity, availability, efficiency and integration of care (Spreeuwen-berg \& Pop, 2000). Goals like efficiency of care set the boundaries of shared care. That is, if shared care does not lead to improvement of care within existing budgets, or a reduction of costs while maintaining quality, it is not worthwhile to implement shared care models.

In shared care models often some form of substitution of tasks is applied. Tasks can be substituted either horizontally or vertically (Nancarrow \& Borthwick, 2005; Van Wijmen et al., 2000). Horizontal substitution represents the transfer of care among equally qualified professionals, while vertical substitution is the transition of care from a higher to a lower qualified professional, for example from a physician to a specialized nurse. Vertical substitution occurs in several health care sectors (Adams, 2004; Nancarrow \& Borthwick, 2005; Norris, 2001; Stevens et al., 2007). 
In the design of organizational innovations one should keep in mind that innovations should fit the needs of the involved parties, including the patients. Innovations also should be supported by the professionals and the professional bodies to which these professionals belong. It is also important that innovations are manageable, and that they include sufficient education, training and support. Finally, organizational innovations should fit the current working standards.

By assessing in an early stage, for different subgroups, which barriers might impede implementation of an innovation, and which facilitators might enhance implementation, patients and professionals can be involved in the process of designing the innovation. This may advance that the above mentioned preconditions, such as the fitting of needs of involved parties and having the support of the professionals, are met. It may improve expectations towards the innovation and hence facilitate the professionals' and patients' will to change their behavior (Grol, 1997).

Generally, implementing organizational innovation in health care is not easy, especially when innovations require better collaboration among disciplines, changes in patients' behavior or changes in the organization of care (Grol et al., 2007). Determining potential barriers and facilitators is an important step in an implementation process (Grol, 1997; Grol \& Grimshaw, 2003; Robertson et al., 1996; Van Bokhoven et al., 2004; Wensing et al., 1999). Ideally, possible barriers and facilitators should be examined before strategies to implement the change are developed (Bosch et al., 2007; Grol, 1997). That is, insight into barriers and facilitators will help to design the most effective implementation strategy. Therefore, potential barriers and facilitators should be repeatedly measured in an innovation process, starting at a very early stage and possibly continuing when the innovation is implemented. Various theories and frameworks for change exist that mention a multitude of factors that may affect successful implementation of change (Grol \& Wensing, 2004; Robertson et al., 1996). One of these frameworks is that of Cabana et al. (1999), which focuses on the individual professional. In this framework barriers are classified into three main categories: barriers related to knowledge (familiarity, awareness), barriers that affect attitudes (agreement, self-efficacy, motivation, outcome expectancy) and external barriers (patient factors, guideline factors, environmental factors).

Ideally, innovations should not diffuse widely before there is sufficient evidence on their safety and efficiency (Battista, 1989). However, in the real world there is often little evidence available. In general organizational innovations can be designed in different ways, which makes it difficult to perform large trials to collect evidence. Moreover, organizational innovation is often a social process, which makes classical randomized experiments less appropriate (Winkens \& Klazinga, 2000). Evaluation of an organizational innovation generally requires implementation of the innovation, and when agreements among professionals are made in a trial, it may well be difficult to undo these changes. Also, collecting data is expensive, and often not enough budget is available to remove all of the existing uncertainty (Hadley, 2000). The latter is connected with the fact that there is not enough knowledge on which additional evidence would be most valuable. Such knowledge would be helpful to allocate research budgets in the most efficient way. 


\section{Health technology assessment}

Health Technology Assessment (HTA) seeks to inform health policy makers by examining medical, social, ethical and economic implications of technologies in health care (Banta, 1982; Platform HTA, 2001). HTA can be seen as a bridge between the scientific evidence and policy decision-making (Battista \& Hodge, 1999). The term HTA is increasingly used instead of medical technology assessment to emphasize that technology assessment is not confined to new drugs or diagnostic or screening activities in health care, but includes the organization of care and its infrastructure (Platform HTA, 2001).

Characteristics of HTA are that it is interdisciplinary and scientific, that it is society and future oriented, that different alternatives are compared and that it is aimed at decisionmaking (Habbema, 1989). Aspects of research within different disciplines (i.e. medicine, epidemiology, law, ethics, economics and sociology) are synthesized to inform policy makers on whether or not to implement innovations in health care. Health technology assessment is an iterative process, and the assessment needs to continue throughout the life of a technology (Tugwell et al., 1986). This iterative process is illustrated by the Technology Assessment Iterative Loop (TAIL), which is represented in Figure 1.1.

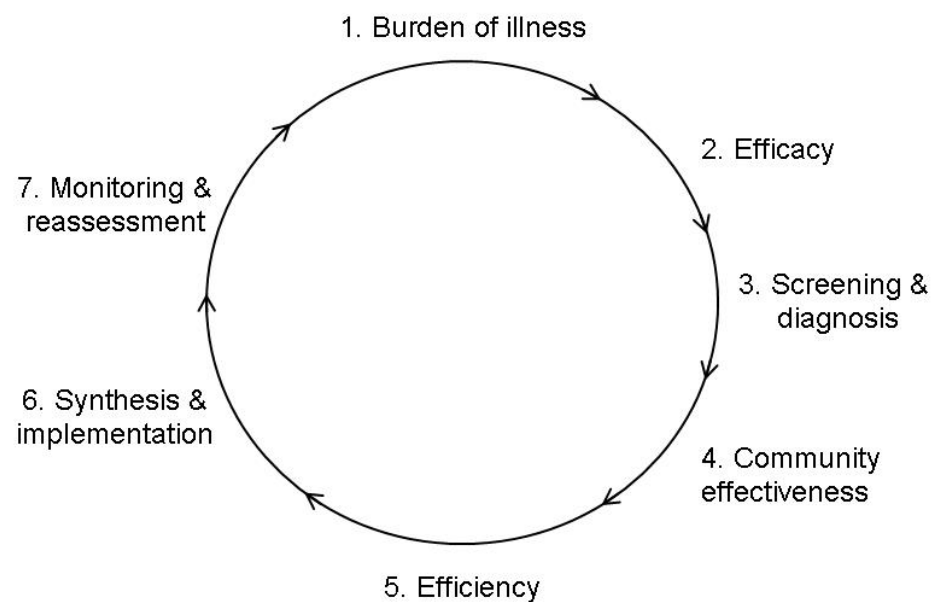

Figure 1.1 The Technology Assessment Iterative Loop (TAIL) (Tugwell et al., 1986)

First, information on the burden of illness is collected to identify the technologies with the greatest potential for reducing the burden of illness. Next, the efficacy of the technology is assessed. Subsequently it is important to identify those persons who will benefit from the efficacious technology, and to examine how well the technology will work in the field. Hereafter the trade-off between the costs and consequences of the technology is examined. The sixth step integrates feasibility, estimated community effectiveness and efficiency to 
make recommendations for implementation of the technology. The TAIL emphasizes that the assessment of a health technology is not finished when the technology is implemented, but that the technology should be continuously monitored and reassessed. By assessing whether the technology has reduced the burden of illness one will eventually arrive at the first step in the cycle, whereupon the cycle will be repeated.

HTA is most often applied to evaluate curative innovations, for example in a pharmaceutical context. In the Netherlands an extensive guideline exists on pharmacoeconomic research, as conclusive evidence needs to be generated before a new drug can be introduced to the market (College voor Zorgverzekeringen, 2006). This conclusive evidence is not required for organizational innovations. Hence, organizational innovations or technologies related to 'care' have rarely been the focus of health technology assessment (Groot \& Maassen van den Brink, 2006). It is questionable whether HTA in its current form is appropriate for evaluating organizational innovation. As mentioned earlier, it can be difficult to collect conclusive evidence on efficacy or community effectiveness of an organizational innovation, and organizational innovation requires implementation before it can be evaluated (Winkens \& Klazinga, 2000). This indicates that the TAIL may not be directly applicable to organizational innovations such as shared care.

A frequently used tool for HTA is the economic evaluation, in which two or more alternatives are compared in terms of both costs and consequences (Drummond et al., 2005). Recently, there has been increasing attention to patient-based health technology assessment (Bridges \& Jones, 2007). The next paragraphs describe these topics and discuss potential methodological issues when evaluating organizational innovations.

\section{Economic evaluation}

Economic evaluation is the 'comparative analysis of alternative courses of action in terms of both their costs and consequences' (Drummond et al., 2005). Hence, the basic tasks of an economic evaluation are to identify, measure, value and compare the costs and consequences of the alternatives that are being considered. There are four types of economic evaluation, which differ in the way the consequences of an intervention are expressed (Drummond et al., 2005). These four types are the cost-minimization analysis, the cost-effectiveness analysis, the cost-utility analysis and the cost-benefit analysis. In a cost-minimization analysis (CMA) only the costs of the alternatives are compared. A CMA is performed when the alternatives are equally effective, but its use has been criticized as it is rarely an appropriate method of analysis (Briggs \& O'Brien, 2001). In a cost-effectiveness analysis (CEA) the incremental effectiveness of an intervention is quantified and compared with its incremental costs. Effectiveness can be measured using various measures of outcome, such as years of life saved, episode-free days or percentage cholesterol reduction. A cost-utility analysis (CUA) is quite similar to a CEA, but in a CUA the measure of effectiveness is the quality adjusted life year (QALY). QALYs are a combined measure of life expectancy and quality of life. Quality of life is expressed in a utility score, ranging from 0 to 1 , where 0 represents death and 1 represents perfect health. Use of such a generic outcome measure allows for comparisons across different interventions and health 
care sectors. Utility scores can be measured either directly or indirectly. The three most widely used direct measures are the visual analogue scale (VAS), the standard gamble and the time trade-off (Drummond et al., 2005). However, only the standard gamble is based directly in the fundamental axioms of utility theory. As directly measuring utility is rather time-consuming and complex, indirect measures are increasingly used to elicit utility scores. Indirect measures make use of pre-scored multi-attribute health status classification systems. Examples of such systems are the EuroQol 5D (The EuroQol Group, 1990), the Health Utilities Index (Feeny et al., 1995) and the Short Form 6D (Brazier et al., 2002).

A final type of economic evaluation is the cost-benefit analysis (CBA), where both costs and consequences are expressed in monetary terms (Drummond et al., 2005). Expressing both costs and consequences in monetary terms facilitates the calculation of an intervention's net benefit (benefits minus costs). This gives the decision-maker a single measure, where a positive net benefit (indicating higher benefits than costs) would indicate the intervention to be worthwhile. CBA is not frequently used though, as assigning monetary values to health outcomes is difficult and controversial (Anell \& Norinder, 2000). In health care, monetary valuations often are assessed through stated preferences (Drummond et al., 2005). Stated preferences can be elicited with contingent valuation. The contingent valuation method involves directly asking individuals what they would maximally be willing to pay for a good or intervention, or how much compensation they would minimally require for loosing it, in a hypothetical market. The resulting willingness to pay (WTP) or willingness to accept (WTA) respectively is a measure of how much the respondent values the good or intervention.

An alternative method to measure stated preferences is conjoint analysis. Conjoint analysis is developed in mathematical psychology and marketing, and is increasingly used in health care (Louviere et al., 2000; Ryan \& Farrar, 2000; Ryan \& Gerard, 2003). It is grounded in random utility theory and based on the principle that complex decisions are not based on a single criterion or factor, but on several factors 'considered jointly'. Any intervention can be described by its attributes, and the extent to which an individual values an intervention depends on the levels of these attributes (Louviere et al., 2000; Ryan \& Farrar, 2000). In conjoint analysis, respondents are presented with various alternative descriptions of an intervention, each of which represents the attributes at different levels. Respondents are asked to rank the various alternatives (ranking), to rate them (rating) or to choose their most preferred (Louviere et al., 2000). The latter is referred to as discrete choice experimentation (DCE), and is increasingly used in health care (Ryan, 2004; Ryan \& Farrar, 2000; Ryan et al., 2001). When a cost attribute is included in the experiment, the willingness to pay or the willingness to accept compensation for a unit change in an attribute can be calculated. Subsequently, each alternative set of change in the levels of the attributes can be given a monetary value.

There is increasing attention to the incorporation of patient perspectives in HTA (Bridges \& Jones, 2007; Platform HTA, 2001; Ryan \& Farrar, 2000). That is, we should not just give them a seat at the decision-making table, but rather scientifically incorporate patient preferences in HTA (Bridges \& Jones, 2007). Conjoint analysis is a useful tool to scientifically measure patient preferences. Much of the appeal of using conjoint analysis to 
elicit patient preferences lays in that it enables us to understand the trade-offs between the attributes. This can for example provide valuable information for health care workers deciding between alternative ways of organizational innovation.

\section{Methodological issues in outcome measurement}

There are a number of methodological issues regarding the measurement of the consequences of an intervention for use in an economic evaluation. First, an important advantage of using a generic outcome measure such as the QALY is that it allows for comparisons across different interventions and health care sectors. Different utility measures however tend to lead to different utility scores (Barton et al., 2005; Bosch \& Hunink, 2000; Brazier et al., 2004; Conner-Spady \& Suarez-Almazor, 2003; Espallargues et al., 2005; Feeny et al., 2004; Kaplan et al., 2005; Kopec \& Willison, 2003; Longworth \& Bryan, 2003; Marra et al., 2004; Marra et al., 2005; O'Brien et al., 2003; Oostenbrink et al., 2002; Petrou \& Hockley, 2005).

In both the Netherlands and the United Kingdom pharmaco-economic guidelines advocate the use of cost-utility or cost-effectiveness analyses (College voor Zorgverzekeringen, 2006; National Institute for Clinical Excellence, 2004). QALYs focus on health gain and thus on the effects of cure, and much less on the effects of care. As QALYs are limited to health outcomes, it is doubtful whether a QALY is an appropriate outcome measure when evaluating different forms of the organization of care. When evaluating organizational innovation one should probably look broader than to health outcomes alone.

The cost-benefit analysis potentially allows a broader evaluative space than CUA, as it expresses consequences in monetary terms. Despite the widespread use of contingent valuation to measure monetary values, there are some methodological controversies. One of these controversies is which type of question should be asked (Diener et al., 1998; O'Brien \& Gafni, 1996). Four types of questions exist to directly measure WTP or WTA: the open-ended, payment scale, bidding game and discrete-choice format (Frew et al., 2003; O'Brien \& Gafni, 1996; Smith, 2000). Different question formats have been found to elicit different results, but there is no consensus on which method elicits the most valid results (Diener et al., 1998; O'Brien \& Gafni, 1996; Smith, 2000). Another methodological controversy is that numerous contingent valuation studies have found that WTA exceeds WTP (Horowitz \& McConnell, 2002; O'Brien et al., 2002). This disparity between WTA and WTP has large implications, as the choice of format may influence the results of a costbenefit analysis, and hence may alter policy recommendations. Whether one should always use WTP (Arrow et al., 1993; Bayoumi, 2004), or use WTP when respondents potentially gain utility from implementing the intervention and WTA when respondents potentially lose utility from implementing the intervention (Drummond et al., 2005; O'Brien \& Gafni, 1996) remains unclear.

An advantage of using conjoint analysis to measure monetary values is that conjoint analysis does not directly ask respondents to express an amount of money. Hence, strategic behavior or protest answers are less likely to occur. However, because of some methodological issues the inclusion of a cost attribute to elicit monetary valuations with 
conjoint analysis should be done with caution (Hanley et al., 2003; Mclntosh, 2006; Ratcliffe, 2000; Slothuus Skjoldborg \& Gyrd-Hansen, 2003). Additionally, it is unknown whether the disparity between WTA and WTP also occurs in conjoint analysis.

\section{Integrating evidence and handling uncertainty}

Economic evaluations can be based on a randomized trial, in which data on resource use and effectiveness are collected simultaneously (Drummond et al., 2005). However, as mentioned before, in organizational innovations randomized trials are often not appropriate. Therefore, in organizational innovations data on resource use and effectiveness are generally not only scarce, but also fragmented. As a result, economic evaluations need to draw on evidence from a range of sources, and the evidence is often surrounded by considerable uncertainty (Claxton et al., 2002).

Decision-analytic modeling is a tool in economic evaluation to synthesize evidence (Weinstein et al., 2003). It can be used to combine data from different sources to inform decision making under conditions of uncertainty (Briggs et al., 2006; Fenwick et al., 2006). In a decision-analytic model, costs and outcomes are simulated, and the effect of an intervention on these outcomes can be investigated. This can be done by using a decision tree, a Markov model or Discrete Event Simulation (Briggs et al., 2006). When input parameters are uncertain, this uncertainty can be incorporated in the model by assigning a range of values to this parameter. One can also model different alternative designs of an innovation, to compare them to each other and the current organization. Additionally, when using a decision-analytic model one can assess the value of additional research, and specifically what type of additional evidence would be most valuable. It can therefore assist in making a twofold decision: 1) whether or not an intervention should be implemented, and 2) whether it is worthwhile to perform additional research to reduce the uncertainty surrounding the first decision (Claxton et al., 2002). 


\section{The case of hearing aid provision}

Hearing impairment is a frequently occurring health problem, with an estimated prevalence of $15 \%$ in the western adult population (Davis, 1995; Johansson \& Arlinger, 2003). Hearing impairment is positively related with age (Davis, 1995; Johansson \& Arlinger, 2003). The ageing of the population will therefore lead to increasing numbers of hearing impaired persons.

There are two types of hearing losses: conductive and sensorineural hearing losses (Cummings, 2005). A conductive hearing loss occurs when sound is not conducted efficiently through the outer ear canal to the eardrum and the middle ear. This type of hearing loss is associated with several conditions such as otitis externa, earwax or otitis media, , and can often be medically or surgically corrected. A sensorineural hearing loss occurs when there is damage to the inner ear or to the nerve pathways from the inner ear to the brain. It is mostly a permanent loss, which cannot be medically or surgically corrected, thus resulting in a chronic disease. Sensorineural hearing loss is caused by ageing or noise exposure, and hearing aids are the only option for rehabilitation. Sensorineural hearing losses are more common than conductive hearing losses and account for approximately $90 \%$ of all hearing losses (Parmet et al., 2007).

Hearing loss can adversely affect a person's social participation, emotional and behavioral wellbeing, and hence one's quality of life (Barton et al., 2005; Dalton et al., 2003; Mulrow et al., 1990). Hearing impaired persons can benefit from using a hearing aid. The use of hearing aids improves social, emotional and communication functions and reduces depression (Appollonio et al., 1996; Joore et al., 2003a; Mulrow et al., 1990). Hearing aid fitting is found to be both an effective (Larson et al., 2000; Mulrow et al., 1990) and a costeffective (Joore et al., 2003b) intervention. Despite the potential benefit, only approximately 20 to $25 \%$ of all persons with hearing problems are fitted with hearing aids (Duijvestijn et al., 1999; Popelka et al., 1998; Stephens et al., 2001). Hearing aid uptake can be influenced by several factors, including awareness of the hearing loss, organization of hearing aid provision, stigma and costs (Stephens, 1987; Stephens et al., 2001).

When hearing-impaired persons in the Netherlands seek help for their hearing complaints, they need to visit their general practitioner (GP) first. A GP can refer a person to either an Ear Nose and Throat (ENT) specialist or an Audiological Centre (AC). In an AC a multidisciplinary team of audiologists, psychologists and social workers, managed by a clinical audiologist, offers specialized care for persons with more serious or complicated hearing impairment. Only ENT-specialists and ACs are entitled to prescribe hearing aids. Based on this prescription, the hearing aid is generally fitted by a private hearing aid dispenser. When both the dispenser and the patient are satisfied with the fitted hearing aid(s), the patient returns to the prescriber for an evaluation and an approval of the hearing aid fitting, which entitles him to (partial) reimbursement of the hearing aid(s). In the Netherlands, patients with a hearing loss exceeding $35 \mathrm{~dB}$, averaged over 1, 2 and $4 \mathrm{kHz}$ in the better ear, were entitled to a maximum reimbursement by their health insurance of $€ 657,50$ per hearing aid in 2007 . This organization of hearing aid provision will be 
referred to as the 'referral pathway' or 'current pathway' throughout this thesis, and is illustrated by the solid lines in Figure 1.2.

The fact that a small proportion of hearing-impaired adults have a conductive hearing loss where management other than hearing aid fitting is required does not mean that all hearing-impaired persons need to see an ENT-specialist or AC (Swan \& Browning, 1994). Therefore, increasing attention is paid to shared care in hearing aid provision. Since 2002, a number of health insurance companies in the Netherlands decided that some of the tasks of ENT-specialists and ACs can be substituted to hearing aid dispensers. This shared care model permits direct hearing aid provision by dispensers, without prescription or approval of an ENT-specialist or AC. This model will be referred to as the 'direct pathway' or shared care in hearing aid provision and is illustrated by both the solid and dashed lines in Figure 1.2. Alternative shared care models are also possible, such as introducing a triage at the private dispenser, but maintaining the evaluation at the ENT-specialist or AC, or maintaining the initial assessment at the GP and ENT-specialist or AC, but omitting the evaluation of the hearing aid fitting by an ENT-specialist or AC.

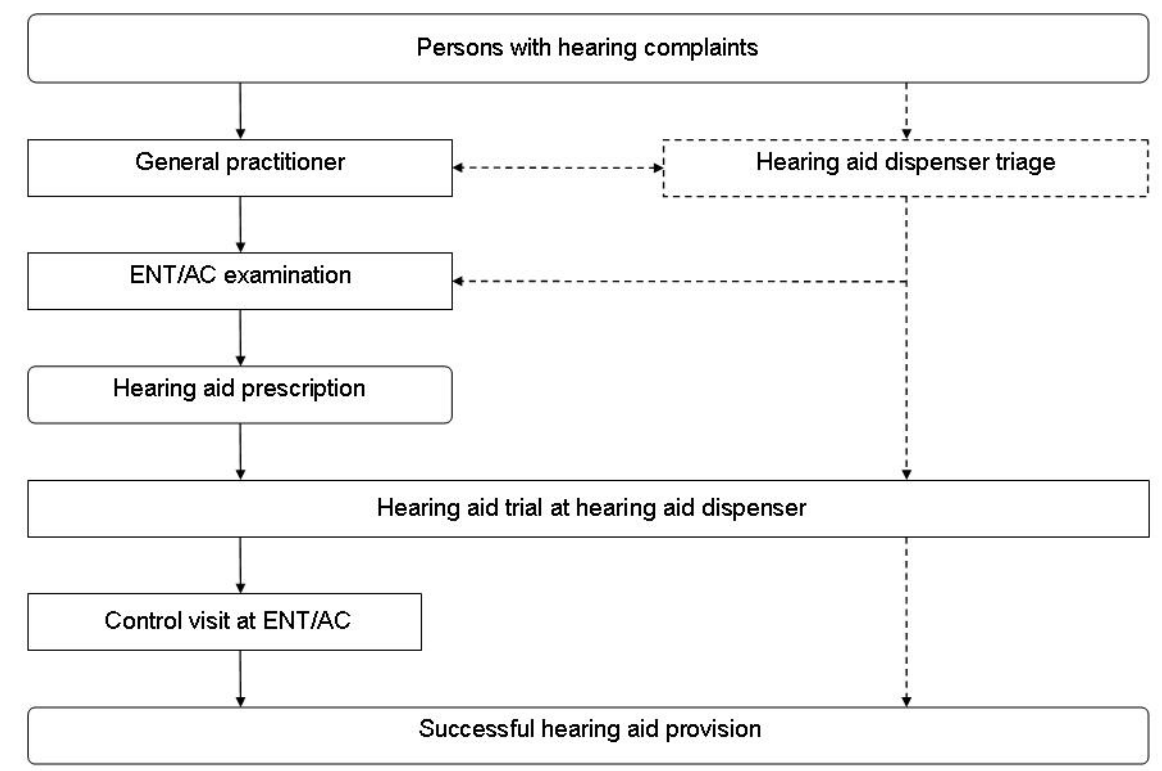

Figure 1.2 Graphical representation of the referral pathway (solid lines) and the direct pathway (solid and dashed lines)

The main reason for this development was the ageing of the population, which causes a proportional growth of the population with hearing impairment and hence increases health care costs associated with hearing rehabilitation. Additionally, an estimated future shortage of ENT-specialists and the fact that this organization of hearing aid provision may be 
perceived as a barrier for help-seeking, lead to increasing attention to direct hearing aid provision by private dispensers. This induced the Dutch Health Care Insurance Board (CvZ) to grant a multi-centre project regarding the safety, quality and efficiency of direct hearing aid provision for persons aged 50 and older, by private dispensers in the Netherlands (AZOS working group, 2006).

Also in other countries, such as Germany and the UK, increasing attention is paid to the involvement of the private sector in hearing aid provision. In the United Kingdom (UK) direct hearing aid provision without medical care has already proven to be safe (Abdelkader et al., 2004; Koay \& Sutton, 1996; Swan \& Browning, 1994). GPs can refer patients to an ENT-specialist or directly to an audiology department. Patients can also directly visit an audiology department (The British Society of Hearing Aid Audiologists, 2005). Unfortunately this has led to a significant range in patient waits from zero to up to three years (British Academy of Audiology, 2006). As a result, the Health Committee recently carried out an inquiry into audiology services in England, with particular interest in the role of the private sector (Health Committee, 2007b), which yielded a wide range of views on the private sector's role in hearing aid provision (Health Committee, 2007a).

Despite the increasing attention little is known about how patients and professionals involved in the care for hearing-impaired persons feel about shared care, and what the relevant issues in the field are. It is therefore unknown whether patients see shared care in hearing aid provision as an improvement. Moreover, little evidence is available on whether private dispensers are capable of distinguishing between persons in need of medical or specialized audiological care (referred to as patients) and persons not in need of medical or specialized audiological care (referred to as clients). It is also uncertain whether hearing aid dispensers will provide good quality hearing aid fittings when these are not evaluated by a third party, and whether shared care will actually save costs. Policy makers therefore require more information before they can make an informed decision on whether or not to allow direct hearing aid provision by private dispensers. Thus, more evidence is needed to allow for evidence-based decision-making regarding shared care in hearing aid provision. 


\section{Objective and research questions}

The objective of the thesis is to use health technology assessment to evaluate whether an organizational innovation, shared care in hearing aid provision, benefits the care for hearing-impaired persons.

The thesis focuses on three aspects of this objective. The first aspect deals with the measurement of barriers and facilitators in an early stage of an organizational innovation process, to become aware of which issues are of importance in the field, and to guide the design process of the innovation. Secondly, outcome measures are considered, both in terms of utilities and monetary valuations. Thirdly, all available evidence is integrated in a decision-analytic model to make a trade-off between the benefits and risks of shared care in hearing aid provision.

We formulated research questions according to these three aspects:

1) What are the expectations of patients and professionals, and potential barriers and facilitators towards shared care in hearing aid provision?

2) How should we measure outcome in shared care in hearing aid provision?

a. How practical, valid, corresponding and responsive are the EuroQol 5D and the Health Utilities Index when measuring health state utility in a population with hearing complaints?

b. How corresponding and valid are the open-ended and payment scale question when directly measuring willingness to pay for a hearing aid?

c. Does the disparity between willingness to accept and willingness to pay occur in a discrete choice experiment?

d. Which preferences do patients have regarding the organization of hearing aid provision?

3) Is shared care in hearing aid provision efficient?

\section{Research methods and design}

To answer this rather wide range of research questions, various research methods are needed. Barriers and facilitators were elicited in a survey using two study populations. The first study population consisted of professionals involved in the care for hearing-impaired persons, being GPs, hearing aid dispensers, ENT-specialists and clinical audiologists. The second study population consisted of persons with hearing complaints aged 50 years and older. The respondents in the second study population were participants in a multi-centre non-controlled prospective evaluation study examining shared care in hearing aid provision versus the referral pathway.

The second research question, regarding outcome measurement in shared care in hearing aid provision, was addressed by three research designs. First, utility scores were measured before and after hearing aid fitting as part of the above-mentioned prospective evaluation study. Secondly, willingness to pay for a hearing aid was directly measured using a questionnaire designed for self-completion. The study population consisted of a 
convenience sample of adult hearing aid users, both first time and experienced hearing aid users, who consulted at the participating hearing aid dispenser practices or ACs. Thirdly, the disparity between WTA and WTP was examined using a discrete choice experiment. Two trained interviewers conducted face-to-face interviews at four private hearing aid dispenser practices, chosen to be representative for the region. Participants were adults with hearing complaints visiting the dispenser. A WTA version and a WTP version of the questionnaire were randomly conducted by both interviewers and were randomly assigned to the respondents in all four practices. The patients' preferences were elicited with the same discrete choice experiment, but only the data from the respondents who received the WTA format were used.

To answer the third research question, whether shared care in hearing aid provision is efficient, all available evidence on this topic was synthesized using a decision-analytic Markov model. The available evidence consisted of data from the prospective evaluation study, as well as a patient cohort study, published literature and expert opinion. From the prospective evaluation study we used the subset of participants of whom we were able to collect sufficient information regarding their hearing aid use, medical status and quality of the hearing aid at the end of the study.

\section{Outline of the thesis}

The thesis starts with an inquiry into the expectations and potential barriers and facilitators towards direct hearing aid provision (Chapter 2). Views on this subject were elicited in a survey among patients with hearing complaints and professionals involved in hearing aid provision.

The second part of the thesis focuses on how one should measure outcome in shared care in hearing aid provision, and consists of Chapters 3 to 6 . In Chapter 3 utility scores are elicited in a population with hearing complaints, before and after hearing aid provision. The differences between preference-based utility measures EuroQol 5D, Health Utilities Index mark II and Health Utilities Index mark III, and their impact on the incremental costeffectiveness ratio for hearing aid fitting are examined. Chapter 4 examines contingent valuation. In contrast to most contingent valuation studies in health care, the amount a person is willing to pay for a hearing aid can be compared to what the person actually has paid for a hearing aid. This makes hearing aid provision a useful subject to examine the validity of contingent valuation elicitation formats. Therefore, Chapter 4 examines the validity of two elicitation formats: the open-ended question and the payment scale. Chapter 5 focuses on the disparity between WTA and WTP, by examining whether this disparity also occurs in discrete choice experiments. It also addresses the question which format should be used, specifically in a DCE regarding shared care in hearing aid provision. Chapter 6 describes the results of a DCE that elicited patient preferences for transferring elements of hearing aid provision from the medical sector to private hearing aid dispensers. 
In the third part of this thesis the available evidence is integrated to make a trade-off between the benefits and risks of shared care in hearing aid provision. In Chapter 7 the evidence on shared care in hearing aid provision is synthesized using a decision-analytic model. The long-term efficiency of different alternative shared care models in hearing aid provision is assessed, and the value of additional research is calculated.

Chapter 8 gives an overview of the thesis. The main findings for each research question are summarized and discussed, followed by the methodological considerations. Finally, recommendations for policy as well as practice are presented, and areas of future research are identified. 


\section{Part I}

Barriers \& facilitators 



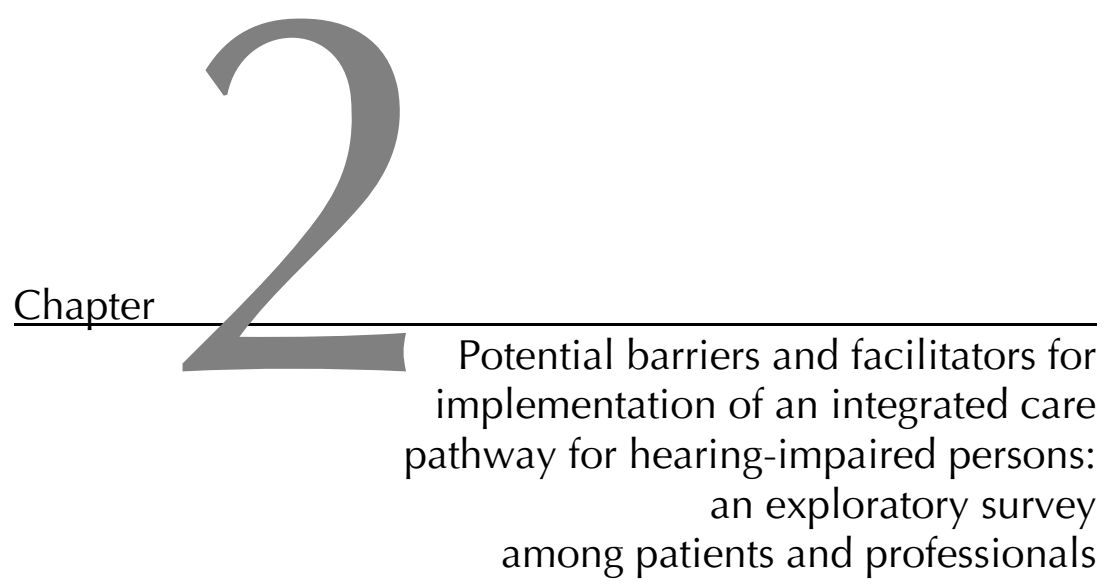

Janneke PC Grutters

Frans van der Horst Manuela A Joore

Hans Verschuure Wouter A Dreschler Lucien JC Anteunis 


\section{Abstract}

\section{Background}

Because of the increasing costs and anticipated shortage of Ear Nose and Throat (ENT) specialists in the care for hearing-impaired persons, an integrated care pathway that includes direct hearing aid provision was developed. While this direct pathway is still under investigation, in a survey we examined expectations and potential barriers and facilitators towards this direct pathway, of patients and professionals involved in the pathway.

\section{Methods}

Two study populations were assessed: members of the health professions involved in the care pathway for hearing-impaired persons (general practitioners (GPs), hearing aid dispensers, ENT-specialists and clinical audiologists) and persons with hearing complaints. We developed a comprehensive semi-structured questionnaire for the professionals, regarding expectations, barriers, facilitators and conditions for implementation. We developed two questionnaires for persons with hearing complaints, both regarding evaluations and preferences, and administered them after they had experienced two key elements of the direct pathway: the triage and the hearing aid fitting.

\section{Results}

On average GPs and hearing aid dispensers had positive expectations towards the direct pathway, while ENT-specialists and clinical audiologists had negative expectations. Professionals stated both barriers and facilitators towards the direct pathway. Most professionals either supported implementation of the direct pathway, provided that a number of conditions were satisfied, or did not support implementation, unless roughly the same conditions were satisfied. Professionals generally agreed on which conditions need to be satisfied. Persons with hearing complaints evaluated the present referral pathway and the new direct pathway equally. Many, especially older, participants stated however that they would still visit the GP and ENT-specialist, even when this would not be necessary for reimbursement of the hearing aid, and found it important that the ENT-specialist or Audiological Centre evaluated their hearing aid.

\section{Conclusions}

This study identified professional concerns about the direct pathway for hearing-impaired persons. Gaps exist in expectations amongst professions. Also gaps exist between users of the pathway, especially between age groups and regions. Professionals are united in the conditions that need to be fulfilled for a successful implementation of the direct pathway. Implementation on a regional level is recommended to best satisfy these conditions. 


\section{Background}

An important step in an implementation process is to determine the barriers that will impede implementation, and the facilitators that will enhance implementation, within different subgroups (Grol, 1997; Grol \& Grimshaw, 2003; Robertson et al., 1996; Van Bokhoven et al., 2004; Wensing et al., 1999). Insight into barriers and facilitators will help to design the most effective implementation strategy. In the Netherlands a new care pathway for hearing-impaired adults is currently under investigation. The present study focuses on potential barriers and facilitators for implementation of this new care pathway.

Most hearing-impaired adults have an age-related sensorineural hearing loss, where hearing aids are the only option for rehabilitation. Until recently, in the Netherlands all hearing-impaired persons who seek help first had to consult their general practitioner (GP) for referral to an Ear Nose and Throat (ENT) specialist or Audiological Centre (AC). In an AC a multidisciplinary team under the direction of a clinical audiologist offers specialized care for the more serious or complicated hearing problems. Only ENT-specialists and ACs were entitled to prescribe hearing aids. Generally, a hearing aid dispenser performed the actual hearing aid fitting. Once a satisfying hearing aid was fitted, the hearing-impaired persons returned to the ENT-specialist or AC for approval of the hearing aid, which entitled them to (partial) reimbursement by their medical insurance. We will refer to this pathway as the care pathway for hearing-impaired persons by referral (Figure 2.1), in short the referral pathway.

Since 2002 however a number of health insurance companies in the Netherlands permit hearing aid reimbursement without a referral, prescription or approval. This allows for direct hearing aid provision by the hearing aid dispenser. One reason for this new care pathway was to reduce health care costs. Approximately $10 \%$ of the general population of western countries is hearing impaired, and this prevalence heavily increases with age (Chorus et al., 1995; Davis, 1995). As a result the current growth of the ageing population causes a proportional growth of the population with hearing impairment. This will raise medical consumption for hearing impairment and, as a consequence, increases the costs of medical care. Another reason for the new care pathway was to reduce the workload of ENT-specialists, since a report was published in 2000 that alerted to a future shortage of ENT-specialists in the Netherlands (Van der Velden \& Hingstman, 2000).

It has been suggested that a proportion of hearing-impaired individuals can safely be fitted with a hearing aid without medical care (Abdelkader et al., 2004; Hawthorne et al., 1991; Koay \& Sutton, 1996; Swan \& Browning, 1994). These individuals are defined as clients throughout the present study. In order to distinguish these 'clients' from the 'patients' who require medical attention or treatment of an AC, a set of criteria was developed by a national body of professionals involved in the care pathway for hearing-impaired persons (Veldhuizen et al., 2002). The criteria for referral relate to otoscopy (i.e. skin aberration, fluid in the auditory canal), audiometry (i.e. asymmetrical hearing loss or sudden deafness) and psychosocial aspects (i.e. communication problems at work). This set of criteria is part of a recently developed integrated care pathway for hearing-impaired persons. Integrated care pathways are structured multidisciplinary care plans which detail essential steps in the 
care of patients with a specific problem (Campbell et al., 1998). The new integrated care pathway is referred to as the direct care pathway for hearing-impaired persons including direct hearing aid provision, in short the direct pathway (Figure 2.1). In parts of the United Kingdom, direct hearing aid provision is by now daily routine (The British Society of Hearing Aid Audiologists, 2005). While in the Netherlands the safety and efficiency of the direct pathway are still under evaluation, we found it important to examine whether persons with hearing complaints and professionals support the direct pathway, and what they consider to be barriers and facilitators, in order to anticipate on the implementation process of the direct care pathway.

The objective of this study was therefore to gain insight into the expectations and potential barriers and facilitators towards the direct pathway.

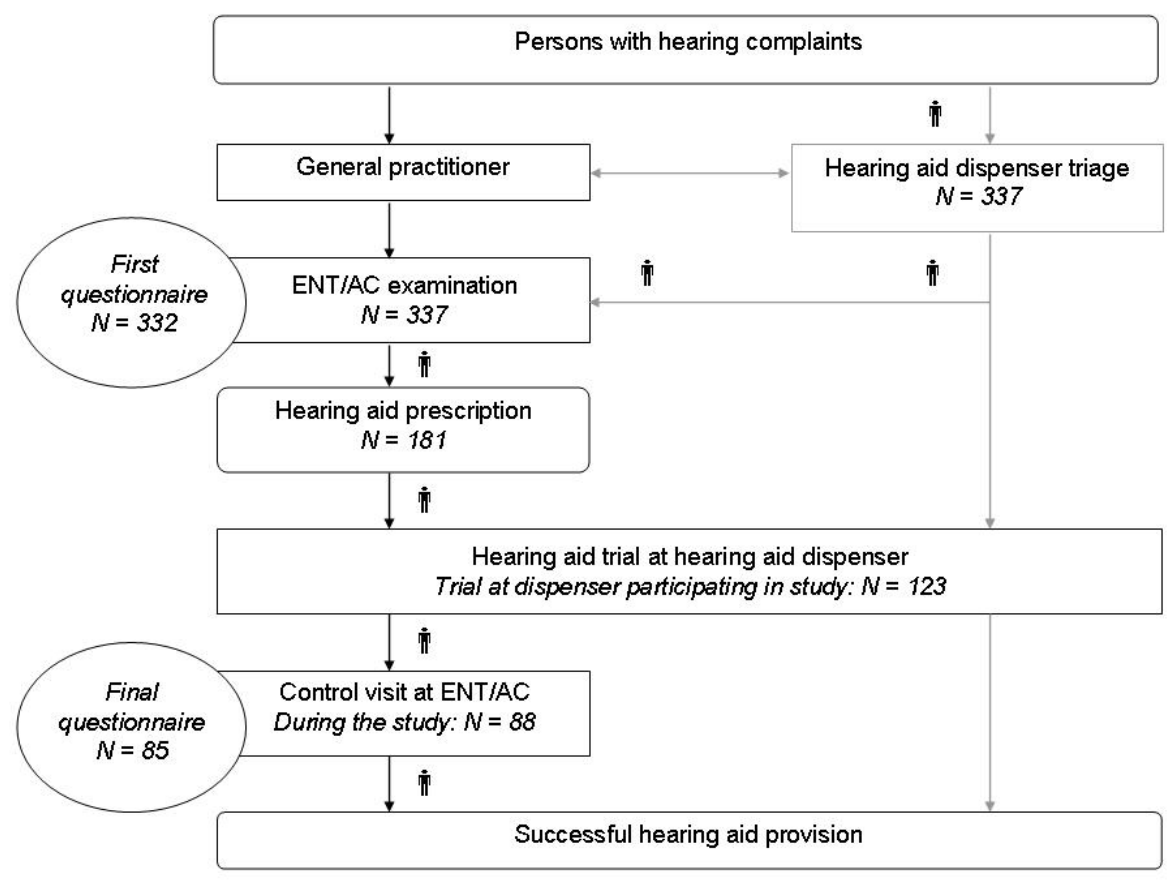

Figure 2.1 Graphical representation of referral pathway (black lines) and the direct pathway (black and grey lines), including the study pathway ( $)$, the study population (italic) and the questionnaire timing (rounds) 


\section{Methods}

\section{Study population}

Two study populations were assessed in the present study. The first study population consisted of members of the health care professions involved in the care pathway for hearing-impaired persons: GPs, hearing aid dispensers, ENT-specialists and clinical audiologists. We approached professionals from three regions in the Netherlands (Amsterdam, Maastricht and Rotterdam), divided over the four professions.

The second study population consisted of persons who consulted for hearing complaints. We included persons of 50 years and older with hearing complaints, who consulted ENT departments and ACs or hearing aid dispensers in the three regions. Persons who had consulted for hearing complaints in the preceding year were considered to be under treatment and were therefore excluded.

\section{Design}

Data for this exploratory survey were collected as part of a multi-centre non-controlled prospective evaluation study examining the direct pathway versus the referral pathway (AZOS working group, 2006). The study was carried out from April 2004 until August 2005 in three regions (Amsterdam, Maastricht and Rotterdam) in the Netherlands. The study was approved by the Medical Ethical Committees of the participating hospitals, and written consent was obtained from all participants. Three teaching hospitals and 11 hearing aid dispensers participated in the study. Since the hospital in Maastricht is the only hospital in its region, it also has a community function. The hospitals in Amsterdam and Rotterdam mainly operate as centers for secondary referral for patients in need of specialized care. The study focused on two key elements of the direct care pathway: distinguishing persons who need medical or specialized audiological care (the patients) from persons who do not need medical or specialized audiological care (the clients), and the quality of the hearing aid provision. For the purpose of the study, participants first visited the hearing aid dispenser for a triage visit (dispenser-triage, Figure 2.1). At the triage visit, the hearing aid dispenser examined whether the participant was in need of medical or specialized audiological care or not and hence should be defined as a patient or client respectively. To perform this examination, the hearing aid dispenser had followed a training program especially designed for this purpose. The main topics of the training program were audiometry and otoscopy. Regardless of the outcome of the triage, all participants subsequently visited the ENT-specialist and AC for a second examination (ENT/ACexamination), to evaluate whether the dispenser had correctly defined the participant as patient or client. If the participant received a prescription for hearing aids, the hearing aid dispenser fitted a hearing aid. Once a satisfying hearing aid fitting was obtained, the participant returned to the AC for a control visit, to evaluate the quality of the hearing aid fitting. 


\section{Framework}

Barriers and facilitators are defined as factors that prevent or enhance, respectively, behavioral change (Wensing et al., 1999). Various theories and models for change point to a multitude of factors that may affect change (Grol \& Wensing, 2004; Robertson et al., 1996). Cabana et al. (1999) developed a framework that focuses on the individual professional. In this framework barriers are classified into three main categories: barriers related to knowledge (familiarity, awareness), barriers that affect attitudes (agreement, selfefficacy, motivation, outcome expectancy) and external barriers (patient factors, guideline factors, environmental factors). We used this framework to structure the barriers and facilitators that health care professionals perceived regarding the implementation of the direct pathway.

In the care pathway for hearing-impaired persons, patient factors play an important role as well. Therefore, patient factors were examined separately in a survey among persons with hearing complaints. Wensing and Elwyn (2004) distinguish three types of patient views: evaluations (reactions to their service experience), preferences (ideas about what should occur) and reports (objective observations). In this study we focused on subjective patient views, and therefore examined evaluations and preferences.

\section{Questionnaire for professionals}

Health care professionals involved in the care pathway (GPs, hearing aid dispensers, ENTspecialists and clinical audiologists) were asked to complete a comprehensive semistructured questionnaire regarding the direct pathway (see Appendix 2.1). First, we paid explicit attention to the outcome expectancies. Professionals were asked on five point Likert scales whether they expected the direct pathway to have positive or negative consequences for different aspects of the hearing aid provision. These six aspects were: diagnosing hearing impairment; indicating a hearing aid; paying attention to medical, audiological and psychological complications; giving objective information about the impairment, treatment options and hearing aids; choice and fine-tuning of the hearing aid; and the quality of the ear moulds. Next, using open questions the professionals were asked to report if they thought the direct pathway was an improvement for some hearingimpaired persons, and for whom, and whether it was a deterioration for some hearingimpaired persons, and for whom. They were also asked to report possible risks and benefits of the direct pathway. Finally, professionals were asked if the direct pathway should be implemented and, if applicable, under which conditions.

\section{Questionnaire for persons with hearing complaints}

Two questionnaires for persons with hearing complaints, designed for self-completion, were developed. The questionnaires were administered after the participants had experienced the two key elements of the direct pathway: the triage and the hearing aid fitting. The first questionnaire was administered directly after the ENT/AC-examination (see Appendix 2.2). Two questions concerned evaluations of the hearing aid dispenser: whether 
participants had confidence that the hearing aid dispenser, who had received additional training, was able to distinguish between clients and patients, and whether they were satisfied with the way the hearing aid dispenser behaved towards them. Regarding their preferences, we asked the participants if they would be inclined to obtain a hearing aid sooner when they didn't have to visit their GP and an ENT-specialist or AC first. Also, they were asked whether they would still visit the GP and ENT-specialist or AC if this was not a prerequisite for (partial) reimbursement of the hearing aid. The questions were answered on five point Likert scales and participants were also given the option that they did not know.

As our study population consisted of persons with hearing complaints, and not only hearing aid applicants, not all participants had a hearing aid fitted. The subset of persons with hearing complaints who were fitted with a hearing aid within the study period completed the final questionnaire after the control visit at the AC (see Appendix 2.3). Both the referral pathway and the direct pathway were described and participants were asked to value both pathways with a grade between 1 and 10, with 10 being the most favorable. After these two evaluative questions, three preference questions were asked on five point Likert scales, including a 'don't know' option. Participants were asked whether they expected that the hearing aid dispenser would fit a good hearing aid without involvement of the ENT-specialist or AC and to report if they found it important that the hearing aid fitting would be advised and evaluated by the ENT-specialist or AC.

We checked the results for differences in sex (male versus female) and age group (below versus above median age) using Mann-Whitney $U$ tests. Differences between the regions (Amsterdam, Maastricht, Rotterdam) were examined with Kruskal Wallis tests, and MannWhitney $U$ tests for post-hoc pairwise comparisons.

\section{Results}

\section{Professional views}

\section{Outcome expectancy}

A total of 60 health care professionals completed the questionnaire regarding their expectations towards the direct pathway. This group consisted of 14 GPs, 16 hearing aid dispensers, 16 ENT-specialists and 14 clinical audiologists.

The professional groups gave significantly different answers on all aspects: diagnosing hearing impairment; indicating a hearing aid; paying attention to medical, audiological and psychological complications; giving objective information about the impairment, treatment options and hearing aids; choice and fine-tuning of the hearing aid; and the quality of the ear moulds (Kruskal Wallis, p-value $<0.01$ ). On average GPs and hearing aid dispensers expected the direct pathway to have positive consequences for all aspects (Figure 2.2). The ENT-specialists expected on average negative consequences for all aspects except the quality of the ear moulds, for which they expected no change. The clinical audiologists expected on average negative consequences for all six aspects. 


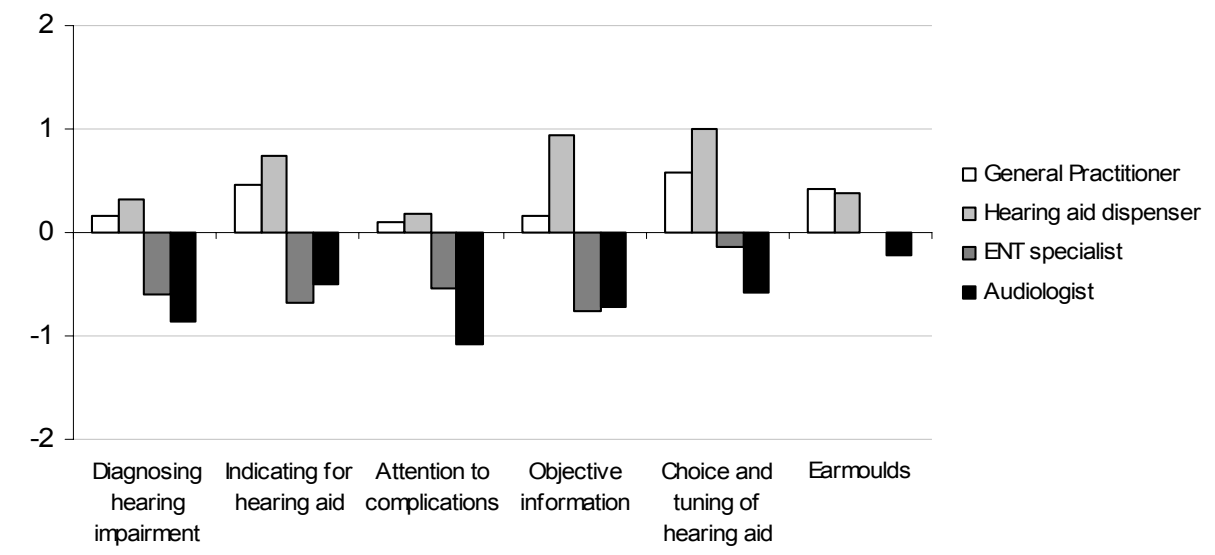

Figure 2.2 Expectations of profession groups towards the direct pathway, elicited with five point Likert scales, ranging from 2 (positive) to -2 (negative)

\section{Barriers and facilitators}

All professionals considered the direct pathway an improvement for a subpopulation of hearing-impaired persons (Table 2.1). Professionals defined this subpopulation as 'older' persons, persons with hearing loss due to ageing only, persons with initial resistance for seeking medical care, and experienced hearing aid users. Almost all professionals (98\%) saw benefits in the direct pathway. These benefits were breaking down barriers, efficiency gain, quicker access to medical care for those in need of it, minimization of costs, reduction in the duration of the pathway, and reduction of the burden on the ENTspecialist, resulting in more time for medical specialist care. Hearing aid dispensers also reported closer collaboration between the involved professions as a benefit.

The majority of professionals (78\%) on the other side considered the direct pathway a deterioration for persons in need of medical care, 'younger' persons, and persons who are dissatisfied with their hearing aid. The majority of professionals (83\%) mentioned the risk of undetected pathology, wrongful indication for a hearing aid or commercialization of care as potential risks of the direct pathway. In addition, GPs emphasized that their role as gatekeeper should be maintained. They reported that a medical cause of the hearing problems should be ruled out first and that diagnostics and treatment should not go hand in hand. By this they meant that the hearing aid dispenser should not both diagnose hearing impairment and provide the hearing aid. Clinical audiologists additionally reported that both the GP and the hearing aid dispenser are not capable of distinguishing adequately between patients and clients. 
Table 2.1 Opinions of professionals on improvement, deterioration, risks and benefits of the direct pathway

\begin{tabular}{lrrrrr}
\hline Profession group & Valid N & $\begin{array}{r}\text { Improvement? } \\
\text { \% Yes }\end{array}$ & $\begin{array}{r}\text { Benefits? } \\
\text { \% Yes }\end{array}$ & $\begin{array}{r}\text { Deterioration? } \\
\text { \% Yes }\end{array}$ & $\begin{array}{r}\text { Risks? } \\
\% \text { Yes }\end{array}$ \\
\hline General practitioner & 14 & $100 \%$ & $100 \%$ & $64 \%$ & $79 \%$ \\
Hearing aid dispenser & 16 & $100 \%$ & $100 \%$ & $56 \%$ & $63 \%$ \\
ENT- specialist & 16 & $100 \%$ & $94 \%$ & $94 \%$ & $100 \%$ \\
Clinical audiologist & 14 & $100 \%$ & $100 \%$ & $100 \%$ & $93 \%$ \\
Total & 60 & $100 \%$ & $98 \%$ & $78 \%$ & $83 \%$ \\
\hline
\end{tabular}

\section{Conditions for implementation}

Ten out of 14 GPs stated that they supported the direct pathway, provided that specific conditions were met (Figure 2.3). These conditions mostly concerned their own role in the pathway: GPs wanted to stay involved in the direct care pathway and emphasized the importance of a good communication between hearing aid dispensers and themselves. Other conditions were adequate training for hearing aid dispensers, that direct hearing aid provision should only be possible for persons of 65 years and older, and that, especially in the beginning, the care should be evaluated frequently.

Two GPs completely supported implementation. One GP reported not to support implementation, unless GP and hearing aid dispenser would work together, and one GP reported absolutely not to support implementation.

Twelve out of 16 hearing aid dispensers reported that they supported implementation, but only when adequate training, strict requirements for hearing aid dispensers, clear criteria for referral, excellent communication between professionals, and good arrangements regarding task division were provided. Four hearing aid dispensers reported to support the implementation completely.

Eight out of 16 ENT-specialists stated to support implementation of the direct pathway, provided that the quality of audiometry and otoscopy would be sufficient, that hearing aid dispensers would not prescribe hearing aids for commercial reasons only, that the professional education for hearing aid dispenser would improve, and that clear criteria for referral, a good evaluation system and a complaints service would be present. Five ENTspecialists reported not to support implementation, unless some of the above mentioned conditions were satisfied. Two ENT-specialists did not support implementation and one ENT-specialist reported to completely support implementation of the direct pathway.

Six out of 14 clinical audiologists stated that they did support implementation, provided that quality would be guaranteed, that there would be a second-opinion possibility, that task division, responsibilities and criteria for referral would be clear, and that hearing aid dispensers would be adequately trained for their new tasks. Four clinical audiologists did not support implementation, except when hearing aid dispenser and GP would recognize all pathology, hearing aid dispensers were sufficiently trained, and a random evaluation of the care pathway would exist. Three clinical audiologists did absolutely not support implementation, in contrast to one who completely supported implementation. 


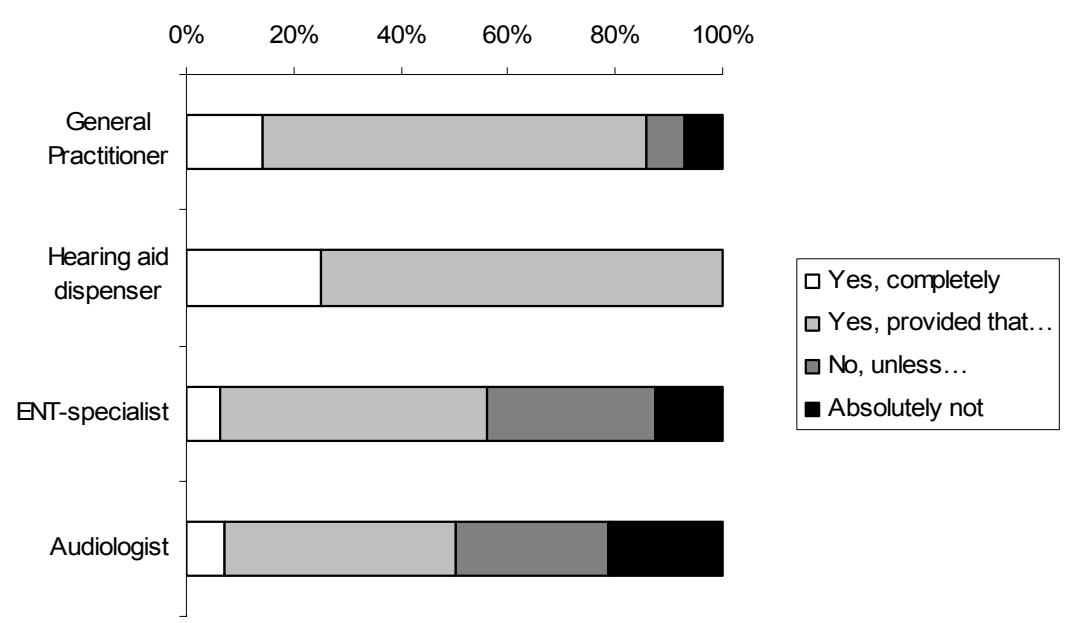

Figure 2.3 Responses to the question whether professionals support implementation of the direct pathway

\section{Views of persons with hearing complaints}

\section{Study population}

Between April 2004 and August 2005, a total of 337 persons with hearing complaints were included in the study and were asked to complete the first questionnaire after the ENT/ACexamination. A total of 332 participants (99\%) completed the questionnaire after the ENT/AC-examination (Figure 2.1). Their mean age was 69.6 years (sd 9.0; median 70) and $60 \%$ were male. The participants were included in Amsterdam $(n=75)$, Maastricht $(n=186)$ and Rotterdam ( $\mathrm{n}=71)$.

Of the 337 participants in the study, 181 (54\%) had a hearing aid fitted. Of them, 88 (49\%) attended the AC for the control visit after the hearing aid trial. Ninety-three participants $(51 \%)$ who had a hearing aid fitted did not show up at the control visit. These participants had not finished their hearing aid trial before the end of the study $(n=35)$, or had a hearing aid fitted at a different dispenser that not participated in the study $(n=58)$ and therefore dropped out of the study. Of the 88 participants who did attend to the control visit, 85 $(97 \%)$ completed the final questionnaire. These 85 participants had a mean age of 70.6 years (sd 7.8; median 71) and $72 \%$ were male. The participants were included in Amsterdam ( $n=14)$, Maastricht $(n=53)$ and Rotterdam $(n=18)$. 


\section{Evaluations}

After the ENT/AC-examination, $89 \%$ of the persons with hearing complaints reported to have confidence in the trained hearing aid dispenser (Table 2.2). Even more respondents $(96 \%)$ were satisfied with the way the hearing aid dispenser behaved towards them.

The subset of persons with hearing complaints who had finished their hearing aid trial and therefore completed the whole pathway $(n=85)$, completed the final questionnaire after the $\mathrm{AC}$ control visit. They graded the referral pathway and the direct pathway equally (7.6 and 7.7 respectively). Both scores ranged from 2 to 10, with a median of 8 for both pathways. The grades were not significantly different (Wilcoxon Signed Ranks Test, p-value 0.896).

Table 2.2 Evaluations and preferences of persons with hearing complaints

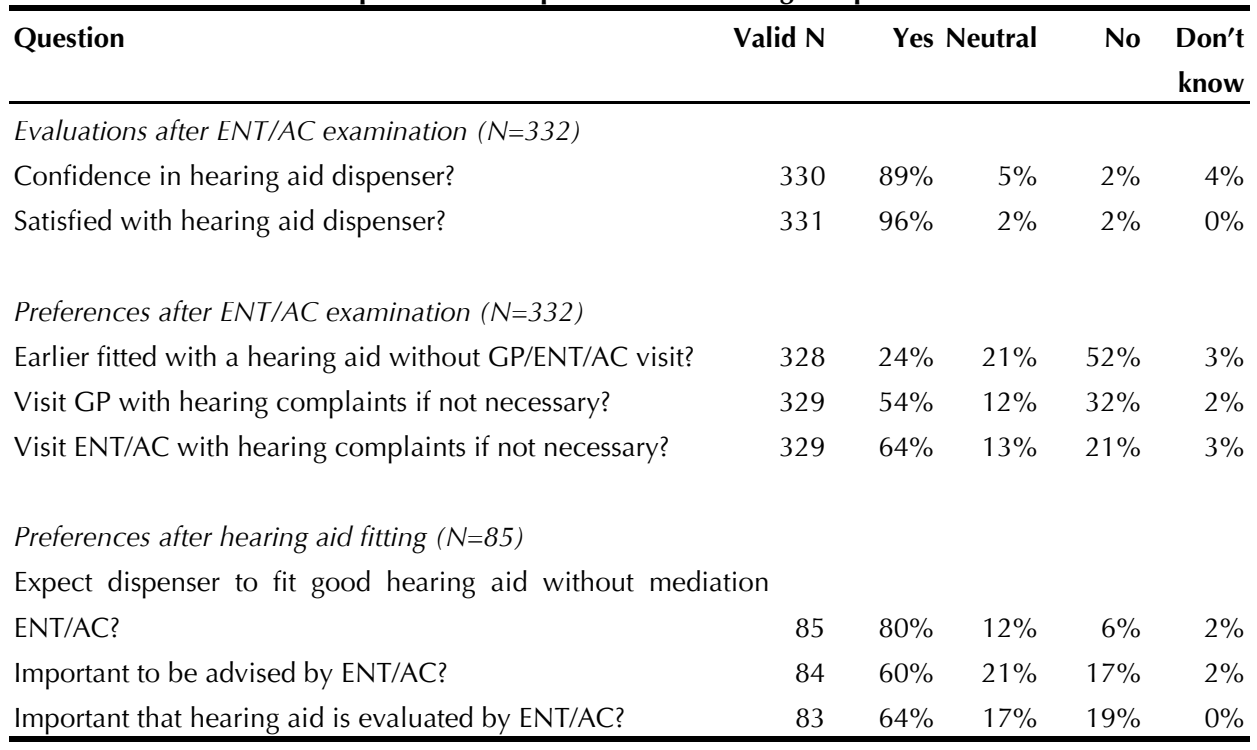

\section{Preferences}

In the first questionnaire, a quarter of the persons with hearing complaints (24\%) reported that they would be inclined to obtain a hearing aid sooner when they didn't have to visit their GP, an ENT-specialist or AC first (Table 2.2). This indicates that a quarter of the participants consider the visits to GP, ENT-specialist and AC as a barrier for hearing aid fitting. Of the participants, $32 \%$ would not visit the GP and $21 \%$ would not visit the ENTspecialist and AC with their hearing complaints when this was not necessary for reimbursement of the hearing aid.

In the final questionnaire, $80 \%$ of the subset of participants who had a hearing aid fitted expected the hearing aid dispenser to perform a good hearing aid fitting, even when the ENT-specialist and AC would not be involved in the process of hearing aid fitting. Even though, a majority of the participants reported that they found it important that an ENT- 
specialist or AC would advise them (60\%), and found it important that the ENT-specialist or AC evaluated the hearing aid fitting (64\%).

\section{Differences between groups}

Additionally, we checked the results for differences in sex (male versus female), age group and region (Amsterdam, Maastricht, Rotterdam). The age groups were defined below and above the median age, being 70 for the group with hearing complaints that completed the first questionnaire, and 71 for the group with a hearing aid fitting that completed the second questionnaire. This resulted in a comparison of persons younger than 70 versus 70 years and older for the first questionnaire, and a comparison of persons younger than 71 versus 71 years and older for the second questionnaire.

No statistically significant differences were found between men and women. There were differences in preferences between the age groups. Of the group of persons aged 70 years and older, $69 \%$ would still visit the ENT-specialist or AC, even if this was not necessary for reimbursement of the hearing aid, versus $58 \%$ of those younger than 70 years. This difference is statistically significant (Mann-Whitney $U, p$-value 0.041). Also, statistically significantly more persons of 71 years and older (79\%) than persons younger than $71(49 \%)$ found it important that an ENT-specialist or AC evaluated their hearing aid fitting (MannWhitney $U$, p-value 0.005).

We also found differences in preferences for the regions, specifically in whether respondents would still visit their GP when this was not necessary (Kruskal Wallis Test, pvalue 0.005). Pairwise comparison showed that in Maastricht (60\%) and Amsterdam (57\%) statistically significantly more persons stated that they would still visit the GP for their hearing complaints than in Rotterdam (37\%) (Mann Whitney $U$, p-values 0.001 and 0.019).

\section{Discussion}

This exploratory study provides insight in the barriers and facilitators of implementing the direct care pathway for hearing-impaired persons. Hearing aid dispensers and GPs had on average positive expectations towards the direct pathway, while ENT-specialists and clinical audiologists had on average negative expectations. Also more ENT-specialists and clinical audiologists found the direct pathway a deterioration and stated possible risks, and they were somewhat more reluctant to implement the direct pathway. Besides concerns about safety and quality, a possible explanation for this is that in the direct pathway, ENTspecialists and clinical audiologists will lose domain in the health care for hearingimpaired persons. They have to hand over part of their work to the GP and hearing aid dispenser. They may feel that their professional authority will decline if they are relegated more to the role of specialist, seeing patients only on referral from hearing aid dispensers and GPs. Hearing aid dispensers on the other hand experience an expansion of their tasks in the care for hearing-impaired persons, as they will have greater autonomy in the direct pathway. This makes hearing aid dispensers a so-called 'encroaching profession'. Also in 
other health care settings established professions develop strategies to protect their boundaries, while encroaching professions try to expand their work areas (Adams, 2004; Nancarrow \& Borthwick, 2005; Norris, 2001; Stevens et al., 2007).

In 2005 an updated report regarding the labor market of ENT-specialists was published. This report concluded that the predicted shortage of ENT-specialists was tackled, and that a sufficient number of ENT-specialists will be available in the near future (Hansen et al., 2005). These findings possibly play a role in the expectations of especially the ENTspecialists, since they may be more reluctant to hand over a number of tasks when their workload will not be as heavy as they expected it to be. The latter also happened in the field of orthopaedic surgeons, who decided to discard a number of unwanted tasks when there was an undersupply of orthopaedic surgeons, and wished to reclaim these tasks when an oversupply arose (Nancarrow \& Borthwick, 2005).

Although negative expectations exist, the results of this study show that most health care professionals either supported implementation of the direct pathway, provided that a number of conditions are satisfied, or did not support implementation, unless roughly the same conditions are satisfied. The professionals generally agreed on which conditions need to be satisfied. In general, these conditions are: good communication between the professionals involved in the direct care pathway, adequate training for the hearing aid dispensers, frequent evaluation, clear criteria for referral, clear division of tasks, a second opinion possibility, and a complaints service.

Before implementing the direct care pathway, all parties involved (GPs, hearing aid dispensers, ENT-specialists, clinical audiologists and hearing-impaired persons) should reach a consensus on the criteria for referral and the division of tasks. Regardless of these criteria and the division of tasks, it should always be possible for hearing-impaired persons to visit the ENT-specialist or AC for a second opinion. Furthermore, the parties must agree on clear requirements regarding the training of the hearing aid dispensers, and these requirements must concur with the referral criteria and task description of the hearing aid dispensers. Next, a complaints service should be facilitated, possibly by the patients' association. Especially in the beginning the direct care pathway should be evaluated carefully. Good communication between professionals is of great importance, but this is probably not easy to realize. Regional implementation may be part of the solution, as in small regions it is less difficult to create a basis of trust among the professionals involved. When the professionals and hearing-impaired persons make agreements on a regional level, they will get to know each other better. As a result this might improve their communication and their confidence in the other professionals' capabilities.

All four groups of professionals stated that the direct pathway had risks as well as benefits. When we apply the framework of Cabana et al. (1999) to the barriers mentioned by the professionals, all are barriers that affect attitudes. The GPs mentioned barriers related to lack of agreement, being the importance of their gatekeeper role and that diagnostics and treatment should not go hand in hand. Most barriers however are related to lack of outcome expectancy. This is clear from the negative expectations of ENT-specialists and clinical audiologists (Figure 2.2) towards the direct pathway, but also shows in the risks that were stated by all professionals. Professionals stated the risk of undetected pathology, the 
risk of wrongful indications for a hearing aid, and the risk of fitting hearing aids for commercial reasons only. Some professionals considered the GP and hearing aid dispenser not capable of distinguishing between persons who need medical or specialized audiological care (patients) and persons who do not need medical or specialized audiological care (clients). If the direct pathway will be implemented, it is important to first consider and appropriately influence the attitudes of the professionals. This may be more successful on a regional level, since closer collaboration between the professionals will probably result in more confidence in each other's capabilities. Information with regard to the safety of the direct pathway is likely to be crucial. Although barriers related to knowledge were not mentioned in this study, when implementing the direct pathway it is important to make sure that everyone involved has sufficient knowledge of the pathway. The professionals did not perceive any external barriers, but we examined these separately by asking persons with hearing complaints for their evaluations and preferences. Due to the study design, approximately half of the participants fitted with a hearing aid could not be asked to complete the final questionnaire. We checked whether age and region in this group were different from the group that did complete the final questionnaire, as these characteristics were found to influence preferences. Since there was no statistically significant difference, we had no reason to believe that the low response rate of the final questionnaire influenced the results.

Although participants evaluated both pathways equally and had confidence in the hearing aid dispenser, many participants stated that they would still visit the GP and ENT-specialist, even when this would not be necessary for reimbursement. This means that after implementation of the direct pathway, clients may still choose to visit the ENT-specialist, bypassing the direct pathway. It is therefore important that persons with hearing complaints are well informed about the direct pathway and are involved in the implementation process.

A larger proportion of older participants stated that they would visit the ENT-specialist or $A C$, even when this was not necessary for reimbursement of the hearing aid, and found it important that the ENT-specialist or AC evaluated their hearing aid fitting. This indicates that older persons are more attached to the opinion of the medical specialist. This difference was not caused by differences in hearing aid experience. The implication of this difference is that especially older clients may bypass the direct care pathway by still visiting the ENT-specialist or AC for their examination and control visit. Older persons therefore may need special attention in the implementation process. Differences in preferences were found between the regions, which is probably due to the variety in care pathways for hearing-impaired persons that currently exist among regions. Since 2002 a number of health insurance companies already permit some form of direct hearing aid provision without consultation of the involved professionals. This makes the current care for hearingimpaired persons diffuse. The differences between the regions and the current diffuse forms of care for hearing-impaired persons argue for implementation on a regional level. The different types of hospitals may have caused the difference in participant numbers included in the study. Maastricht included more participants than Rotterdam and Amsterdam, 
possibly because the hospital in Maastricht also has a community function and sees more persons that are not referred by another hospital.

Since multidisciplinary collaboration and task substitution play an important role in many other integrated or shared care pathways (Campbell et al., 1998; Grone \& Garcia-Barbero, 2001; Mur-Veeman et al., 2001; Stevens et al., 2007), the barriers and conditions for implementation found in this study are likely to apply to other integrated care pathways as well.

While the direct care pathway is still under investigation, we have already obtained valuable information regarding support of the direct care pathway and potential barriers and facilitators. Barriers and facilitators to change are often examined after or during implementation (Cabana et al., 1999; Espeland \& Baerheim, 2003; Foy et al., 2005; Haagen et al., 2005). By determining barriers in an earlier stage, we can use the information to develop the implementation process and to increase the chance of successful implementation.

\section{Conclusion}

This exploratory study identified professional concerns about the direct care pathway for hearing-impaired persons. It is clear that gaps in expectations amongst GPs, hearing aid dispensers, ENT-specialists and clinical audiologists exist. It is also clear that gaps in expectations exist among the users of the care pathway, especially with older persons demonstrating a preference for the traditional pathway. Also, persons from different regions where currently different pathways exist demonstrated different preferences. Despite these differences and despite the fact that implementation of the direct pathway is not yet broadly based, professionals are united in the conditions that need to be fulfilled for a successful implementation of the direct pathway. It is important that these conditions are met before implementing the direct pathway in the Netherlands. Implementation on a regional level is recommended to best satisfy the stated conditions. 


\section{Appendix 2.1 Questionnaire for professionals}

What consequences do you think that the new care pathway for hearing-impaired persons will have with regard to the following aspects:

1. Diagnosing hearing impairment?

$$
-2 \square-1 \square
$$

Negative consequences

$0 \square$

$1 \square$

Positive consequences

2. Indicating a hearing aid?

$\begin{array}{llll}-2 & -10 & 0 & 10\end{array}$

Negative consequences

Positive consequences

3. Paying attention to medical, audiological and psychological complications?

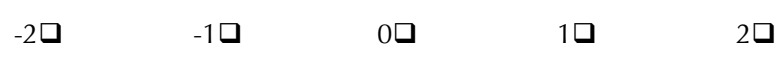

Negative consequences Positive consequences

4. Giving objective information on hearing impairment, treatment options and hearing aids?

$\begin{array}{llll}-2 & -10 & 0 & 0\end{array}$

Negative consequences Positive consequences

5. Choice and fine-tuning of the hearing aid?

$\begin{array}{llll}-2 & -10 & 0 & 0\end{array}$

Negative consequences Positive consequences

6. The quality of the ear moulds?

$\begin{array}{llll}-2 & -10 & 0 & 10\end{array}$

Negative consequences Positive consequences

7. Do you think the new care pathway will be an improvement for a subset of persons?

- Yes, that is...

$\square$ No, because...

8. Do you think the new care pathway will be a deterioration for a subset of persons?

Yes, that is...

$\square$ No, because...

9. Do you think the new care pathway will have possible risks?

Yes, that is...

- No, because...

10. Do you think the new care pathway will have possible benefits?

Yes, that is...

- No, because...

11. Do you think that the new care pathway should be implemented?
- Yes, completely
口 Yes, provided that...
口 I don't know, because...
口 No, unless...
口 Absolutely not 


\section{Appendix 2.2 First questionnaire for persons with hearing complaints}

With the following statements we would like to find out how you think about the care for persons with hearing complaints. Could you please indicate whether you agree with the following statements?

1. I have confidence that the trained hearing aid dispenser is able to determine whether referral to an ENT-specialist or AC is necessary

2. I am satisfied with the way the hearing aid dispenser behaved towards me

3. I am inclined to obtain a hearing aid sooner, when I don't have to visit my GP and an ENT-specialist or AC first

4. I would still visit my GP for my hearing complaints if this was not necessary for reimbursement of the hearing aid

5. I would still visit the ENT-specialist or AC for my hearing complaints if this was not necessary for reimbursement of the hearing aid

\section{Neither}

agree

Totally Quite nor Quite Totally Don't

agree agree disagree disagree disagree

know
$\mathrm{O}$

$\mathrm{O}$

$\mathrm{O}$

$\mathrm{O}$

$\mathrm{O}$ 


\section{Appendix 2.3 Final questionnaire for persons with hearing complaints}

The care for hearing-impaired persons can be organized in different ways. With the next questions we would like to know what you think about two possible ways of organizing this care.

In the current pathway the hearing-impaired patient first visits the ENT-specialist or the AC. Here he is examined and receives a prescription for a hearing aid. Next, the hearing aid dispenser and the patients start a hearing aid trial, where the patient can try several hearing aids. When the patient and dispenser are satisfied with the hearing aid, the patient visits the ENT-specialist or AC to evaluate the hearing aid.

1. If you were asked to value this pathway with a grade from 1 to 10 , with 10 being the most favorable, what grade would you give?

In the new pathway the hearing-impaired patient first visits the hearing aid dispenser. The dispenser examines whether the patient needs medical care. When necessary the dispenser refers the patient to an ENT-specialist or AC. When the patient is not in need of medical care, the dispenser and patient directly start the hearing aid trial, without prescription. When the patient and dispenser are satisfied with the hearing aid, the patient can go home without having his hearing aid evaluated by an ENTspecialist or AC.

2. If you were asked to value this pathway with a grade from 1 to 10 , with 10 being the most favorable, what grade would you give?

Could you please indicate whether you agree with the following statements?

3. I expect that the hearing aid dispenser will fit a good hearing aid without involvement of the ENTspecialist or AC

4. I find it important that the hearing aid is advised by an ENT-specialist or $\mathrm{AC}$

5. I find it important that the hearing aid is evaluated by an ENT-specialist or $\mathrm{AC}$

\begin{tabular}{|c|c|c|c|c|c|}
\hline & & $\begin{array}{l}\text { Neither } \\
\text { agree }\end{array}$ & & & \\
\hline $\begin{array}{c}\text { Totally } \\
\text { agree }\end{array}$ & $\begin{array}{l}\text { Quite } \\
\text { agree }\end{array}$ & $\begin{array}{c}\text { nor } \\
\text { disagree }\end{array}$ & $\begin{array}{c}\text { Quite } \\
\text { disagree }\end{array}$ & $\begin{array}{l}\text { Totally } \\
\text { disagree }\end{array}$ & $\begin{array}{l}\text { Don't } \\
\text { know }\end{array}$ \\
\hline 口 & ם & 口 & 口 & a & $\mathrm{O}$ \\
\hline ם & ם & ם & ם & ם & $\mathrm{O}$ \\
\hline ם & 口 & ם & ם & ם & $\mathrm{O}$ \\
\hline
\end{tabular}




\section{Part II}

Outcome measurement 



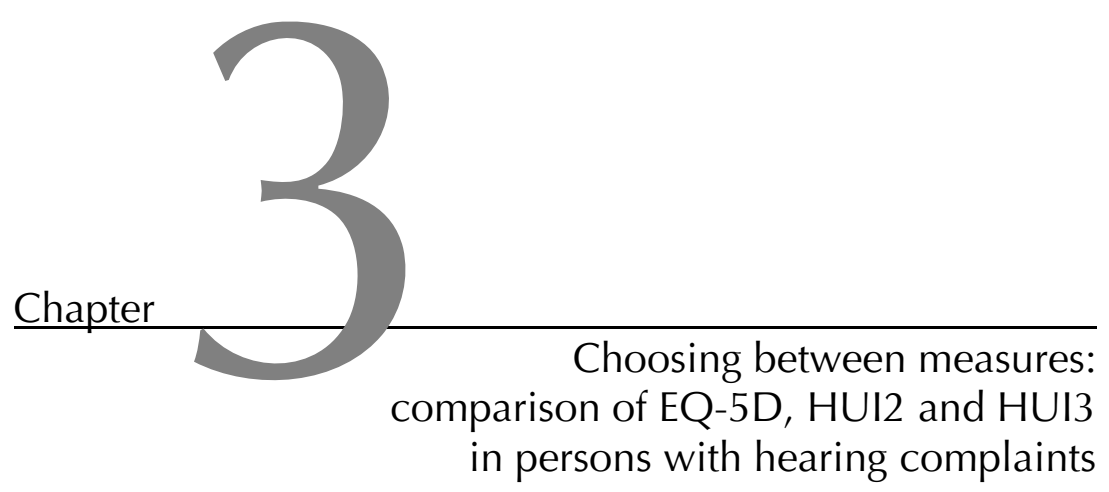

Janneke PC Grutters Manuela A Joore Frans van der Horst Hans Verschuure Wouter A Dreschler Lucien JC Anteunis

Quality of Life Research 2007, 16(8): 1439-1449 


\section{Abstract}

\section{Objectives}

To generate insight into the differences between utility measures EuroQol 5D (EQ-5D), Health Utilities Index Mark II (HUI2) and Mark III (HUI3) and their impact on the incremental cost-effectiveness ratio (ICER) for hearing aid fitting.

\section{Methods}

Persons with hearing complaints completed EQ-5D, HUI2 and HUI3 at baseline and, when applicable, after hearing aid fitting. Practicality, construct validity, agreement, responsiveness and impact on the ICER were examined.

\section{Results}

All measures had high completion rates. HUI3 was capable of discriminating between clinically distinctive groups. Utility scores $(n=315)$ for EQ-5D UK and Dutch tariff $(0.83$; 0.86), HUI2 (0.77) and HUI3 (0.61) were significantly different, agreement was low to moderate. Change after hearing aid fitting $(n=70)$ for HUI2 (0.07) and HUI3 (0.12) was statistically significant, unlike the EQ-5D UK (0.01) and Dutch (0.00) tariff. ICERs varied from $€ 647209$ per QALY for the EQ-5D Dutch tariff to $€ 15811$ per QALY for HUI3.

\section{Conclusion}

Utility scores, utility gain and ICERs heavily depend on the measure that is used to elicit them. This study indicates HUI3 as the instrument of first choice when measuring utility in a population with hearing complaints, but emphasizes the importance of a clear notion of what constitutes utility with regard to economic analyses. 


\section{Introduction}

Hearing loss affects the ability to exchange information and therefore affects a person's quality of life (Dalton et al., 2003). Of the western adult population, approximately $15 \%$ are hearing impaired (Davis, 1995), and for these persons hearing aid use has proven to be effective (Appollonio et al., 1996; Mulrow et al., 1990). The growth of the elderly population has far-reaching implications for auditory health service delivery and expenditure since the prevalence increases heavily with age. As a result, increasingly more economic evaluations are undertaken on interventions such as hearing aid fitting. In economic evaluations health related quality of life (HRQoL) should be measured with a preference-based utility measure (Drummond et al., 2005). Until recently, the benefits of hearing aid fitting had not translated into a statistically significant improvement in health state utility (Bess, 2000; Joore et al., 2003a; Joore et al., 2003b). In 2004 Barton et al. (2004) confirmed this for the EuroQol 5D (EQ-5D), but did find statistically significant utility gain after hearing aid fitting with the Health Utilities Index Mark III (HUI3). This difference may result from differences in the descriptive system, and the way the scoring function is derived.

Also with regard to other conditions, a number of studies have found that different utility measures tend to lead to different utility scores (Bosch \& Hunink, 2000; Brazier et al., 2004; Conner-Spady \& Suarez-Almazor, 2003; Espallargues et al., 2005; Feeny et al., 2004; Kaplan et al., 2005; Kopec \& Willison, 2003; Longworth \& Bryan, 2003; Marra et al., 2004; Marra et al., 2005; O'Brien et al., 2003; Oostenbrink et al., 2002; Petrou \& Hockley, 2005). In general there is need for head-to-head comparisons of utility measures, in order to assess the implications for the interpretation and comparability of economic analyses, especially in conditions where only subtle changes after treatment are expected (Brazier \& Deverill, 1999; Pickard et al., 2005). In these comparisons the comparison of different tariffs should also be incorporated. But most importantly, the impact of the differences between measures on incremental cost-effectiveness ratios (ICERs) should be made apparent. Surprisingly, the latter has not yet received much attention.

The objective of this article is to compare the two most frequently used utility measures in economic analyses (Rasanen et al., 2006) in a Dutch population with hearing complaints. Utility scores derived with the EQ-5D UK tariff (Dolan, 1997), the EQ-5D Dutch tariff (Lamers et al., 2005), the Health Utilities Index Mark 2 (HUI2) and HUI3 (Feeny et al., 1995) are compared to generate further insight into the differences between the measures, and the impact on the ICER for hearing aid fitting. In particular, this article considers:

1) practicality of using the EuroQol and HUI in a population with hearing complaints;

2) construct validity of the EQ-5D UK tariff, EQ-5D Dutch tariff, HUI2 and HUI3;

3) agreement between the EQ-5D UK tariff, EQ-5D Dutch tariff, HUI2 and HUI3;

4) responsiveness of the EQ-5D UK tariff, EQ-5D Dutch tariff, HUI2 and HUI3 after hearing aid fitting;

5) and the impact of on the ICER for hearing aid fitting. 


\section{Methods}

\section{The EQ-5D, HUI2 and HUI3}

The five questions of the EQ-5D descriptive system each represent one dimension of health-related quality of life (mobility, self-care, usual activities, pain/discomfort and anxiety/depression) (The EuroQol Group, 1990). Each question has three levels and the questions together classify persons into one of 243 health states. The commonly used scoring function is based on a British study (EQ-5D UK tariff) (Dolan, 1997), with preferences derived with the time trade-off (TTO) method, in a representative sample of the UK population of 2,997 respondents. The scoring function is additive and the possible range of utility scores is -0.59 to 1.00 . Recently, Lamers et al. (2005) developed a Dutch scoring function for the EQ-5D (EQ-5D Dutch tariff), based on TTO in a sample of 298 respondents, with utility scores ranging from -0.33 to 1.00 .

The 15 questions of the HUI descriptive system classify respondents into either HUI2 or HUI3 health states. The HUI system focuses on health 'within the skin', meaning that they purely focus on impairment and not on the social context of the impairment (Feeny et al., 1995). HUI2 consists of seven attributes (sensation, mobility, emotion, cognition, self-care, pain and fertility), with three to five levels, leading to 24000 possible health states. The optional fertility attribute was not used in the present study. The multiplicative scoring function was derived using standard gamble (SG) and Visual Analogue Scale (VAS) in a random sample of 293 Canadian respondents and utility scores range from -0.03 to 1.00 (Torrance et al., 1996). HUI3 consists of eight attributes (vision, hearing, speech, ambulation, dexterity, emotion, cognition and pain), with five to six levels and leads to 972000 possible health states. The multiplicative scoring function was derived from SG and VAS, in a random sample of the Canadian general population $(n=504)$, resulting in possible utility scores varying from -0.36 to 1.00 (Feeny et al., 2002).

\section{Study population and data collection}

Data were collected as part of a before-after study examining direct hearing aid provision versus provision by referral (AZOS working group, 2006). The study was carried out in 2004-2005 in three regions (Maastricht, Rotterdam and Amsterdam) in the Netherlands. Persons with hearing complaints were recruited from the participating Ear Nose and Throat (ENT) departments, audiological centres and hearing aid dispensers. Both EQ-5D and HUI were administered at the first visit to the ENT department. Questionnaires were completed at the department, and respondents were helped by a trained interviewer if requested. Patients who were fitted with a hearing aid were asked to attend the ENT department for a follow-up visit to evaluate the hearing aid fitting. During the evaluation at the ENT department this subset of patients completed both questionnaires for a second time. Again, questionnaires were completed at the department, and respondents were helped by a trained interviewer if requested. 


\section{Practicality of the questionnaires}

Especially in an elderly population, an important aspect of a utility measure is the ease of completion. The practicality of using the EQ-5D and the HUI in a population with hearing complaints was therefore assessed by the completion rate, using a Wilcoxon Signed Ranks Test to test whether the completion rates were significantly different. Additionally we examined the item non-response.

\section{Construct validity}

In absence of a gold standard to measure health state utility, there is no clear technique to determine the construct validity of utility measures. A way to examine the construct validity is to examine whether utility scores are different for distinctive groups (Gross Portney \& Watkins, 1993; Holland et al., 2004). Other studies have demonstrated differences of quality of life scores by sex (Burstrom et al., 2001; Holland et al., 2004; Lubetkin et al., 2005) and age (Burstrom et al., 2001; Devlin et al., 2000; Kind et al., 1998; Lubetkin et al., 2005). Comparisons were made between EQ-5D UK and Dutch tariff, HUI2 and HUI3 scores by age (above versus below median) and sex. It was expected that persons of a higher age and females have lower utility scores. In addition, the respondents were divided into five clinically distinctive groups, based on their hearing loss and hearing aid use. Hearing loss was defined as the better ear pure tone average (BEPTA) hearing loss for the frequencies 1000, 2000 and $4000 \mathrm{~Hz}$. The groups were: persons who were not entitled to reimbursement of a hearing aid (BEPTA $<35 \mathrm{~dB}$ ); persons who were entitled to reimbursement (BEPTA $\geq 35 \mathrm{~dB}$ ) but did not apply for a hearing aid (non-applicants); first time hearing aid applicants; experienced hearing aid users who were about to have a new hearing aid fitted (re-applicants); and experienced hearing aid users who did not have a new hearing aid fitted.

It was expected that persons with a BEPTA smaller than $35 \mathrm{~dB}$ would have a higher quality of life score than persons in the other four groups, because they are likely to experience less problems with hearing. It was also expected that non-applicants had a higher utility score than first time applicants, since the latter group is expected to experience more hearing complaints, resulting in fitting a hearing aid as a solution for their hearing problems.

Descriptive summary statistics were provided and normality was tested for all data using the Kolmogorov-Smirnov test. Kruskal-Wallis one way ANOVA and pairwise comparison tests (Mann-Whitney $U$ ) were used to explore the differences between the groups.

\section{Agreement}

To assess agreement between the measures, a Wilcoxon Signed-Rank test and a Paired ttest were used to test whether the scores of the EQ-5D (UK and Dutch tariff), HUI2 and HUI3 had the same distribution and mean. Correlations (Kendall's Tau) and the Intra-class Correlation Coefficient (ICC) were computed. The ICC was based on a two-way mixed 
effect model, such that the subject effect was random and the instrument effect was fixed, and computed at the individual patient level. An ICC below 0.75 implies poor to moderate agreement; above 0.75 implies good agreement (Gross Portney \& Watkins, 1993).

\section{Responsiveness}

Responsiveness was tested in the subpopulation with a valid score both before and after hearing aid fitting. Effect size and standardized response mean were calculated. Effect size is the change in score divided by the standard deviation of scores at baseline. Standardized response mean is the change in score divided by the standard deviation of the change in score. Both were interpreted using benchmarks for effect size: 0.20 through 0.49 is interpreted as small, 0.50 through 0.79 as moderate and $\geq 0.80$ as large (Cohen, 1988). Also, a Wilcoxon Signed Ranks Test and a Paired t-test were conducted on the before and after scores. The change in score after hearing aid fitting was tested for differences between first time hearing aid applicants and re-applicants. It was expected that re-applicants had a smaller change in utility, since they already had a hearing aid and therefore expectedly less hearing problems at baseline.

\section{Impact on the ICER}

To illustrate the impact on the ICER, straightforward one year ICERs of hearing aid fitting versus no hearing aid fitting (doing nothing) were calculated. Quality Adjusted Life Years (QALYs) were calculated using the area under the curve method, for the EQ-5D (UK and Dutch tariff), HUI2 and HUI3 (Drummond et al., 2005). Baseline utility scores were used to estimate the effects of 'no hearing aid fitting'. Costs of no hearing aid fitting were assumed to be zero. This was compared to the costs and effects after hearing aid fitting. The costs of hearing aid fitting were calculated prospectively for each respondent, including General Practitioner (GP) visit(s), ENT visit(s) and hearing aid(s). We used standard costs for medical consumption (Oostenbrink et al., 2004), and the actual price of the hearing aid. As costs and utility scores are generally not normally distributed, a non-parametric bootstrap sampling method was used to calculate the 95\% confidence interval around the ICERs (Briggs et al., 1997). Cost-effectiveness acceptability curves for all measures were created to characterize the likelihood that hearing aid fitting will be deemed cost-effective based on the incremental costs and outcomes, for a range of ceiling ratios. Ceiling ratios reflect societies' maximum willingness to pay for a unit of outcome. 


\section{Results}

\section{Practicality}

Of the 337 persons with hearing complaints included in the study, in total 315 (93\%) fully completed both the EQ-5D and the HUI descriptive system at baseline. Each item on the EQ-5D had six missing values, except for mobility $(n=5)$. Regarding the HUI, all questions had six or seven missing values, except for the questions on hearing in a group conversation $(n=12)$, pain and discomfort $(n=10)$, and hearing in a conversation with one other person $(n=8)$. Although completion rates were high for both questionnaires, EQ-5D was fully completed by 328 persons $(97 \%)$, which is significantly more than the 318 persons (94\%) who completed the HUI (P-value 0.012).

Of the 315 persons who completed both EQ-5D and HUI at baseline, 173 persons (55\%) had a hearing aid fitted. Of them, $82(47 \%)$ attended the ENT department for the follow-up visit after hearing aid fitting. Ninety-one respondents (53\%) who had a hearing aid fitted did not show at the follow-up visit because they had not finished their hearing aid fitting before the end of the study ( $n=37$ ), or because they had a hearing aid fitted at a dispenser not participating in the study $(n=54)$, and were therefore lost to follow up. Of the 82 respondents who did attend the follow-up visit, 70 (85\%) fully completed both the EQ-5D and HUI descriptive system. Each item on the EQ-5D had five missing values, except for pain/discomfort $(n=6)$. Regarding the HUI, the hearing questions both had four missing values, and the other questions had four to seven missing values. Seventy-six persons $(93 \%)$ fully completed the EQ-5D, while 71 persons (87\%) fully completed the HUI. This difference is not statistically significant (P-value 0.059).

\section{Construct validity}

Mean age of the respondents was 69.6 years (sd 8.9; median 70), and BEPTA was on average $42 \mathrm{~dB}$. The respondents were divided into groups below 70 years old $(n=156)$ versus 70 years and older $(n=159)$ and male $(n=189)$ versus female $(n=126)$, see Table 3.1. The scores on the EQ-5D, HUI2 and HUI3 were not normally distributed (KolmogorovSmirnov test, P-value 0.000). Only the EQ-5D detected differences in reported health by both age and sex. HUI3 detected a difference in utility between the age groups, HUI2 did not detect any differences.

Furthermore, the respondents were divided into five clinically distinctive groups: BEPTA < $35 \mathrm{~dB}(n=69)$, non-applicants $(n=46)$, first time hearing aid applicants $(n=108)$, reapplicants $(n=65)$, and experienced hearing aid users not applying for a new hearing aid $(n=12)$. The latter group was excluded from the analysis because of the small sample size. Fifteen persons could not be classified into a clinical group because they were lost to follow-up after the first visit.

Based on the EQ-5D and HUI2, no distinction could be made between any of the clinically distinctive groups. A logarithmic transformation was performed on the EQ-5D and HUI2 data to compensate for skewness. Even after transformation, and also when correcting for 
age, sex and BEPTA, no differences were found between the groups. Only HUI3 scores demonstrated a significant difference between the clinically distinctive groups (KruskallWallis; P-value 0.004). More specifically, HUI3 found significant differences between persons with a BEPTA $<35 \mathrm{~dB}$ and first time applicants (Mann-Whitney U; P-value 0.002), and between persons with a BEPTA $<35 \mathrm{~dB}$ and re-applicants (P-value 0.001). HUI3 did not confirm our expectation that non-applicants had significantly higher utility scores than first time applicants. As expected, non-applicants stated less problems on the hearing attribute than first time applicants, but they also stated more problems on the ambulation and pain attributes.

\section{Agreement}

A summary of the utility scores is presented in Table 3.2. Mean utility scores for the population with hearing complaints were higher for the EQ-5D Dutch tariff (mean 0.86; standard deviation 0.18 ) and EQ-5D UK tariff (0.83; sd 0.21) than for the HUI2 (0.77; sd 0.14 ) and HUI3 (0.61; sd 0.24). The differences in mean scores and distributions are statistically significant. The UK and Dutch tariff of the EQ-5D and HUI2 and HUI3 were found to have good agreement, since they were strongly correlated $(0.90 ; 0.71)$ and had an ICC of 0.98 and 0.74 , respectively. The scores on all other measures showed statistically significant, but low correlations and their agreement was moderate to poor (Table 3.3).

Ceiling effects were observed in the EQ-5D UK tariff (Figures 3.1 and 3.2), results were similar for EQ-5D Dutch tariff. As measured with the EQ-5D (both UK and Dutch tariff), $44 \%$ of the respondents reported perfect health, despite their hearing complaints. Measured with the HUI2 or HUI3, less than $1 \%$ of the respondents reported perfect health. For respondents reporting perfect health on the EQ-5D, mean utility scores were 0.83 on the HUI2 (range 0.35-1.00) and 0.71 on the HUI3 (range 0.06-1.00). 


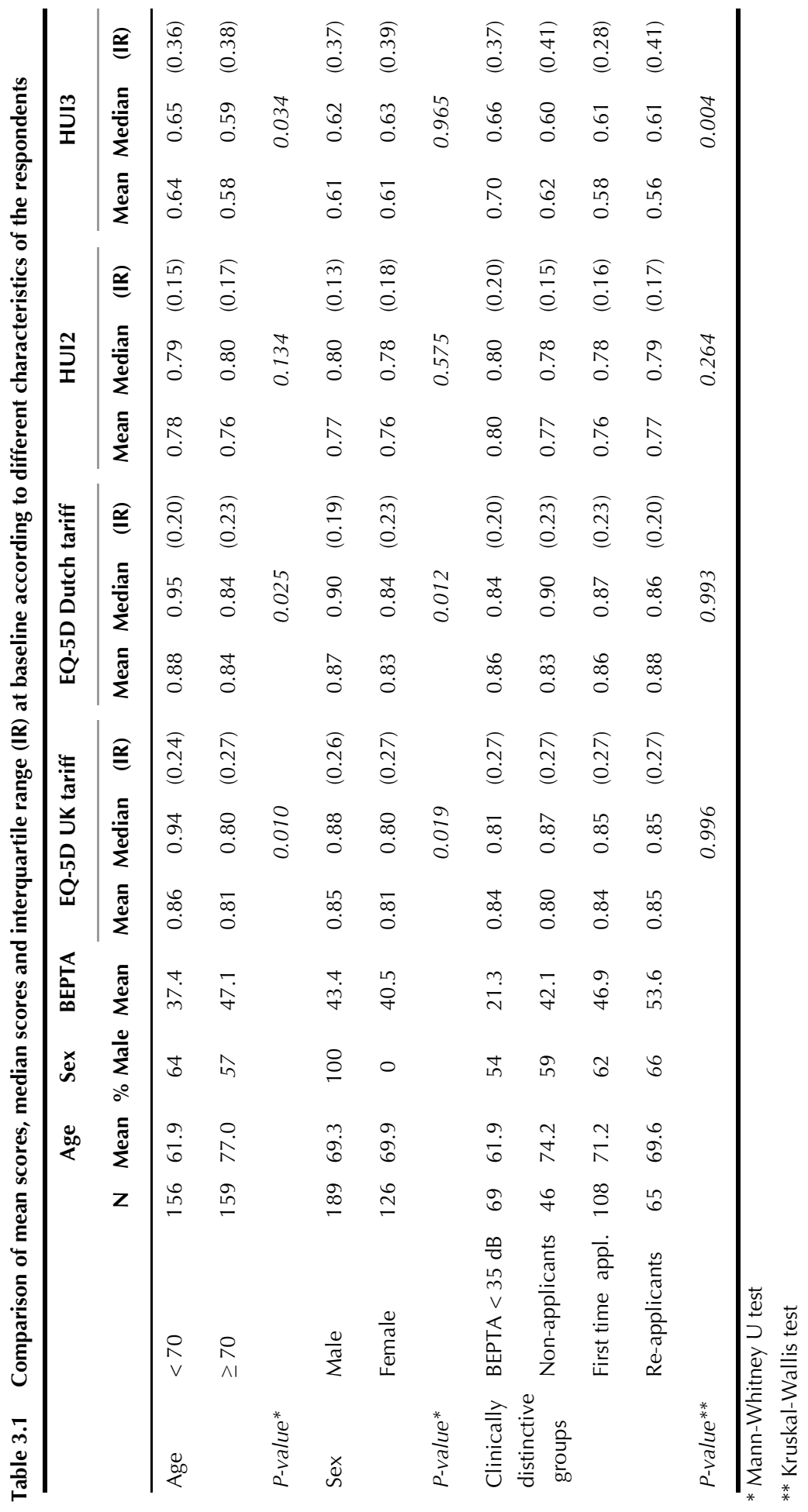


Table 3.2 Utility scores in baseline population with hearing complaints $(n=315)$

\begin{tabular}{|c|c|c|c|c|c|c|}
\hline Measure & Minimum & Maximum & Median* & $\begin{array}{r}\text { Interquartile } \\
\text { range }\end{array}$ & Mean** & $\begin{array}{r}\text { Standard } \\
\text { Deviation }\end{array}$ \\
\hline EQ-5D UK tariff & -0.25 & 1.00 & 0.85 & 0.27 & 0.83 & 0.21 \\
\hline EQ-5D Dutch tariff & -0.03 & 1.00 & 0.86 & 0.19 & 0.86 & 0.18 \\
\hline HUI2 & 0.23 & 1.00 & 0.79 & 0.15 & 0.77 & 0.14 \\
\hline HUI3 & -0.07 & 1.00 & 0.62 & 0.38 & 0.61 & 0.24 \\
\hline
\end{tabular}

* All statistically significantly different: Wilcoxon Signed-Rank test; $p<0.01$

** All statistically significantly different: Paired t-test; $\mathrm{p}<0.01$

Table 3.3 Agreement in the baseline population with hearing complaints $(n=315)$

\begin{tabular}{lrrc}
\hline Pairs of utility functions & Kendall's Tau* & \multicolumn{1}{c}{ ICC } & (95\% Confidence Interval) \\
\hline EQ-5D UK tariff versus HUI2 & $r=0.41$ & 0.51 & $(0.42$ to 0.59$)$ \\
EQ-5D UK tariff versus HUI3 & $r=0.37$ & 0.47 & $(0.38$ to 0.55) \\
EQ-5D UK versus Dutch tariff & $r=0.90$ & 0.98 & $(0.97$ to 0.98) \\
HUI2 versus HUI3 & $r=0.71$ & 0.74 & $(0.68$ to 0.78) \\
HUI2 versus EQ-5D Dutch tariff & $r=0.40$ & 0.51 & $(0.42$ to 0.59) \\
HUI3 versus EQ-5D Dutch tariff & $r=0.36$ & 0.44 & $(0.35$ to 0.53) \\
\hline
\end{tabular}

ICC = Intraclass Correlation Coefficient

* All statistically significant, $\mathrm{p}<0.01$ 


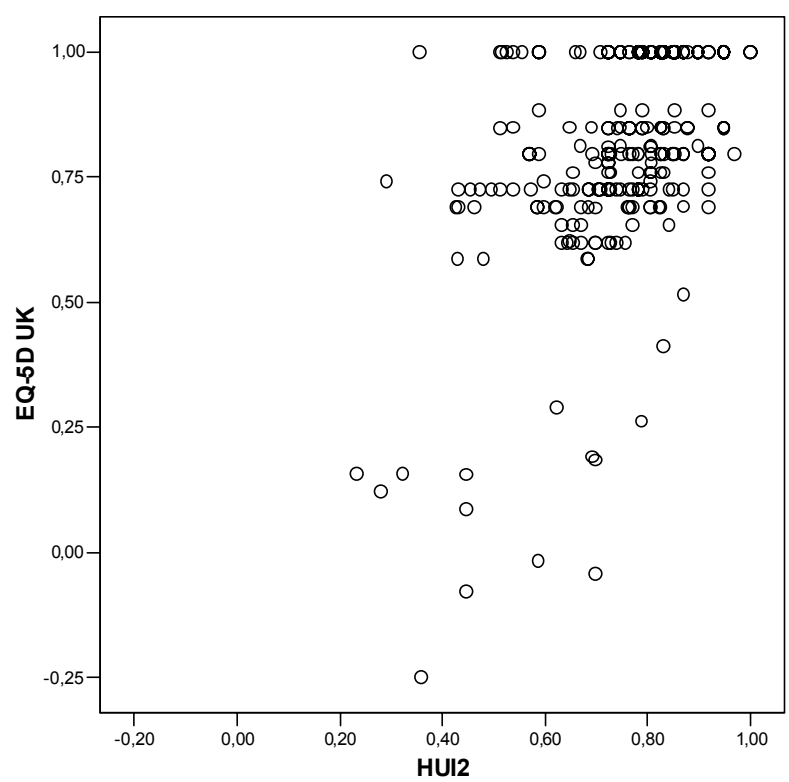

Figure 3.1 Scatterplot of utility scores derived with EQ-5D UK tariff and HUI2

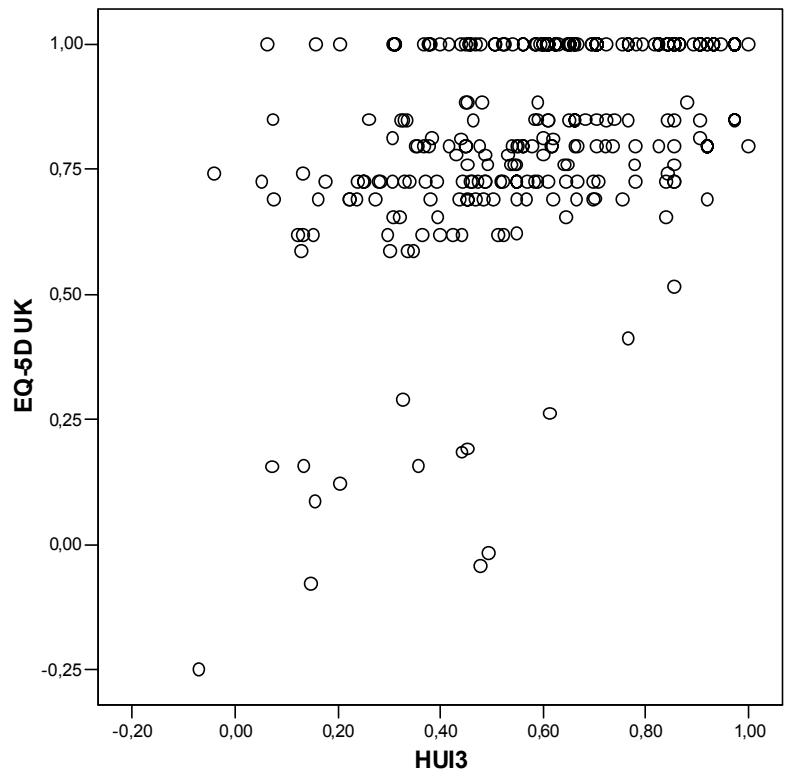

Figure 3.2 Scatterplot of utility scores derived with EQ-5D UK tariff and HUI3 


\section{Responsiveness}

Responsiveness was tested in the subpopulation with a valid score both before and after hearing aid fitting $(n=70)$. The effect size and standardized response mean of the EQ-5D UK $(0.05 ; 0.05)$ and Dutch tariff $(0.03 ; 0.02)$ were less than small. HUI2 and HUI3 were more responsive to change after hearing aid fitting, since both had a moderate effect size $(0.64 ; 0.55)$ and standardized response mean $(0.57 ; 0.66)$.

Mean change in utility after hearing aid fitting (Table 3.4) was highest when measured with the HUI3 (mean 0.12; sd 0.18) and HUI2 (0.07; sd 0.13), while almost no change was measured with the EQ-5D (UK tariff 0.01, sd 0.13; Dutch tariff 0.00, sd 0.12). The change in utility measured with HUI2 and HUI3 is statistically significant (Paired t-test, P-values 0.005 and 0.000 ). No change was observed in any attribute of the EQ-5D (Figure 3.3a). The change in HUI2 utility score after hearing aid fitting occurred in the sensation attribute (Figure 3.3b) and in the HUI3 score in the hearing attribute (Figure 3.3c). Almost no change was observed in any of the other attributes of the HUI2 and HUI3.

The mean change in utility score after hearing aid fitting, when measured with HUI2 and HUI3, was higher for first-time hearing aid applicants $(0.08 ; 0.13)$ than for re-applicants $(0.06 ; 0.10)$. This outcome was in line with our expectations, but is not significantly different.

Table 3.4 Change in health state utility after hearing aid fitting and ICER with $95 \%$ Confidence Interval $(\mathbf{C I})(\mathbf{n}=70)$

\begin{tabular}{|c|c|c|c|c|c|c|c|c|}
\hline Measure & Mean & $\begin{array}{l}\text { Standard } \\
\text { deviation }\end{array}$ & Median & IR & Min & Max & $\begin{array}{r}\text { ICER }^{1} \\
€ / \text { QALY } \\
\end{array}$ & $\begin{array}{l}(95 \% \mathrm{CI}) \\
€ / \text { QALY }\end{array}$ \\
\hline EQ-5D & 0.01 & 0.13 & 0.00 & 0.04 & -0.60 & 0.27 & 286866 & $\left(\right.$ inferior $\left.^{2}-47082\right)$ \\
\hline \multicolumn{9}{|l|}{ UK tariff } \\
\hline EQ-5D & 0.00 & 0.12 & 0.00 & 0.04 & -0.60 & 0.28 & 647209 & $\left(\right.$ inferior $\left.^{2}-61934\right)$ \\
\hline \multicolumn{9}{|c|}{ Dutch tariff } \\
\hline HUI2 & $0.07 *$ & 0.13 & $0.08 * *$ & 0.12 & -0.50 & 0.40 & 25337 & (19 $356-38012)$ \\
\hline HUI3 & $0.12 *$ & 0.18 & $0.13 * *$ & 0.22 & -0.22 & 0.60 & 15811 & $(11664-24654)$ \\
\hline
\end{tabular}

Abbreviations: IR = interquartile range ; Min = minimum ; Max = maximum ; ICER = Incremental CostEffectiveness Ratio; QALY = Quality Adjusted Life Year

* Statistically significant; Paired t-test; $\mathrm{p}<0.01$

** Statistically significantly different: Wilcoxon Signed-Rank test; $\mathrm{p}<0.01$

${ }^{1}$ ICER based on mean scores; ${ }^{2}$ Inferior means higher costs and lower utility 
a.

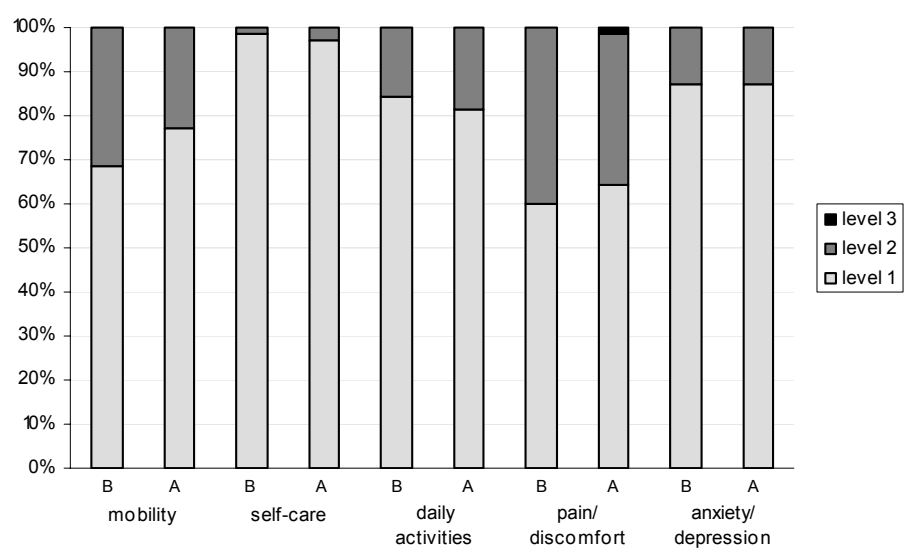

b.

HUl2

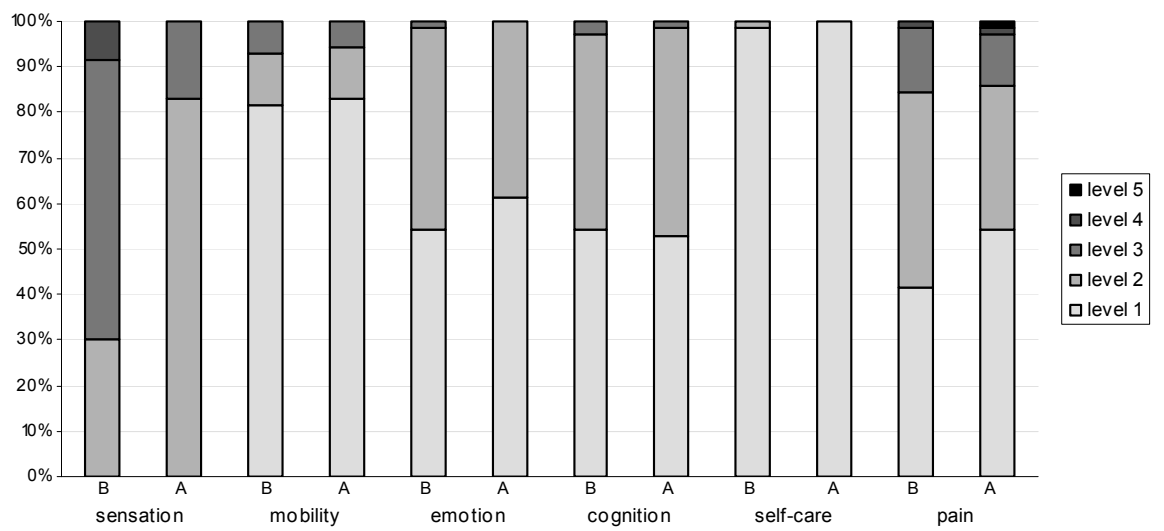

c.

HUl3

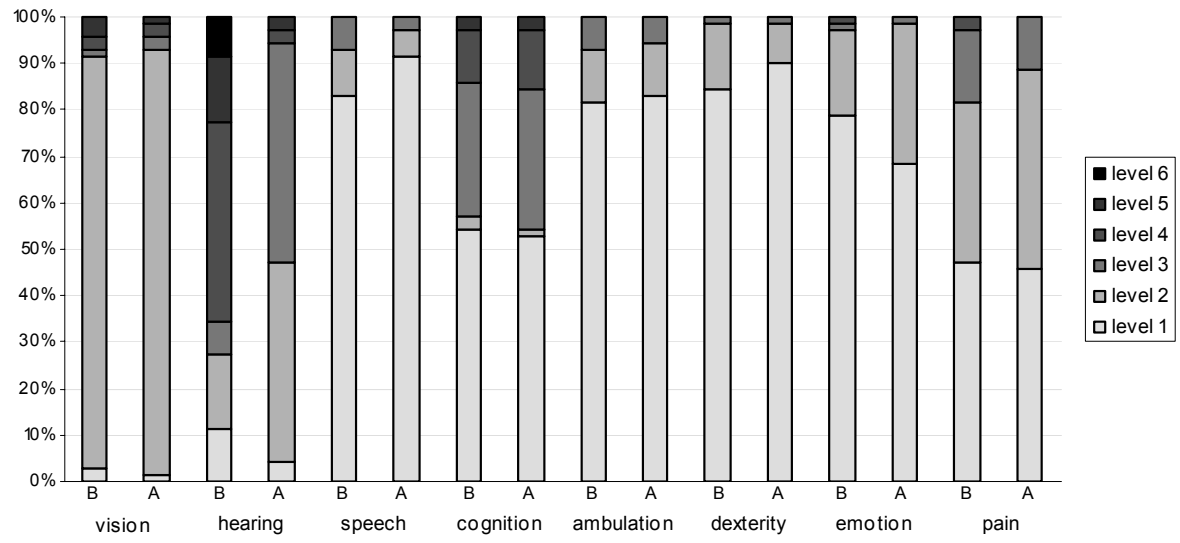

Figure 3.3 Percentage of responses for each level of each attribute before (B) and after (A) hearing aid fitting for a) EQ-5D, b) HUI2 and c) HUI3 


\section{Impact on the ICER}

Mean costs of doing nothing were zero. The mean costs of hearing aid fitting were $€ 1877$. The latter consisted of GP visits ( $€ 37$ ), ENT visits (€ 295) and hearing aid(s) (€ 1545). All hearing aids were digital, and hearing aids were bilaterally fitted in $83 \%$ of the respondents.

This resulted in mean one-year incremental costs of hearing aid fitting versus doing nothing of $€ 1877$. The mean utility gain of 0.01 (sd 0.13 ), measured with the EQ-5D UK tariff, resulted in a ratio of $€ 286866$ per QALY, with a 95\% confidence interval of inferior (higher costs, lower utility) to $€ 47082 / \mathrm{QALY}$. There was a $36 \%$ probability that hearing aid fitting was both more costly and less effective (inferior). The mean utility gain of 0.003 , measured with the EQ-5D Dutch tariff, resulted in an ICER of $€ 647209$ per QALY (95\% confidence interval: inferior to $€ 61934 / \mathrm{QALY}$ ). There was a $42 \%$ probability that hearing aid fitting was inferior. Applying the HUI2 and HUI3, the ICER was € 25337 per QALY (95\% confidence interval: $€ 38$ 012/QALY to $€ 19$ 356/QALY) and $€ 15811$ per QALY (95\% confidence interval: $€ 24654 / \mathrm{QALY}$ to $€ 11$ 664/QALY) respectively. For both measures there was no probability that hearing aid fitting was inferior.

The informal Dutch ceiling ratio of $€ 20000$ per QALY (CBO, 1998) implied that hearing aid fitting was only cost-effective when utility was measured with the HUI3. A costeffectiveness plane with incremental cost and effect pairs for 1000 bootstrap replications, for all measures, is shown in Figure 3.4. Figure 3.5 shows the cost-effectiveness acceptability curves for all measures. 


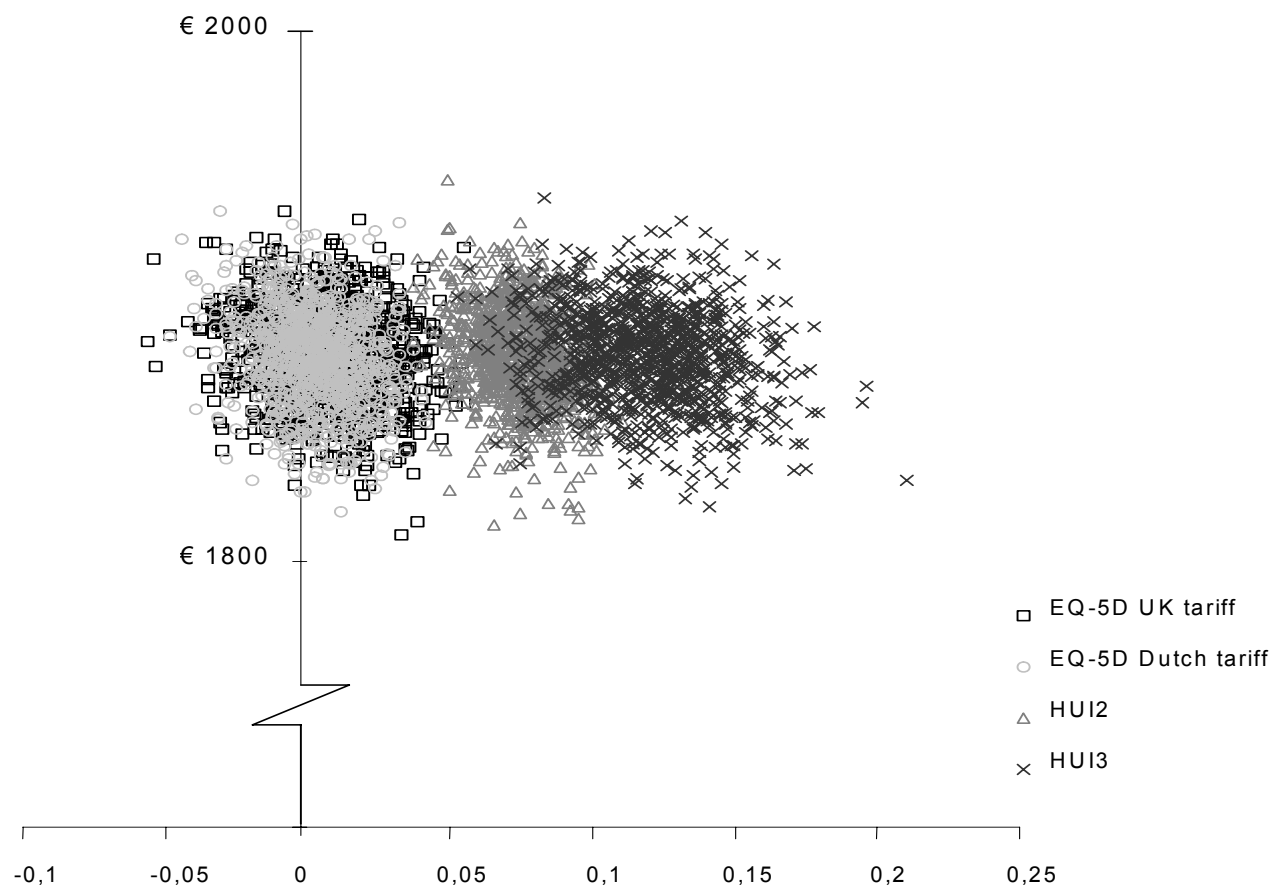

Figure 3.4 Incremental cost-effectiveness ratio for hearing aid fitting (Incremental costs (euro) are displayed at the vertical line, incremental effects (QALYs) on the horizontal line.

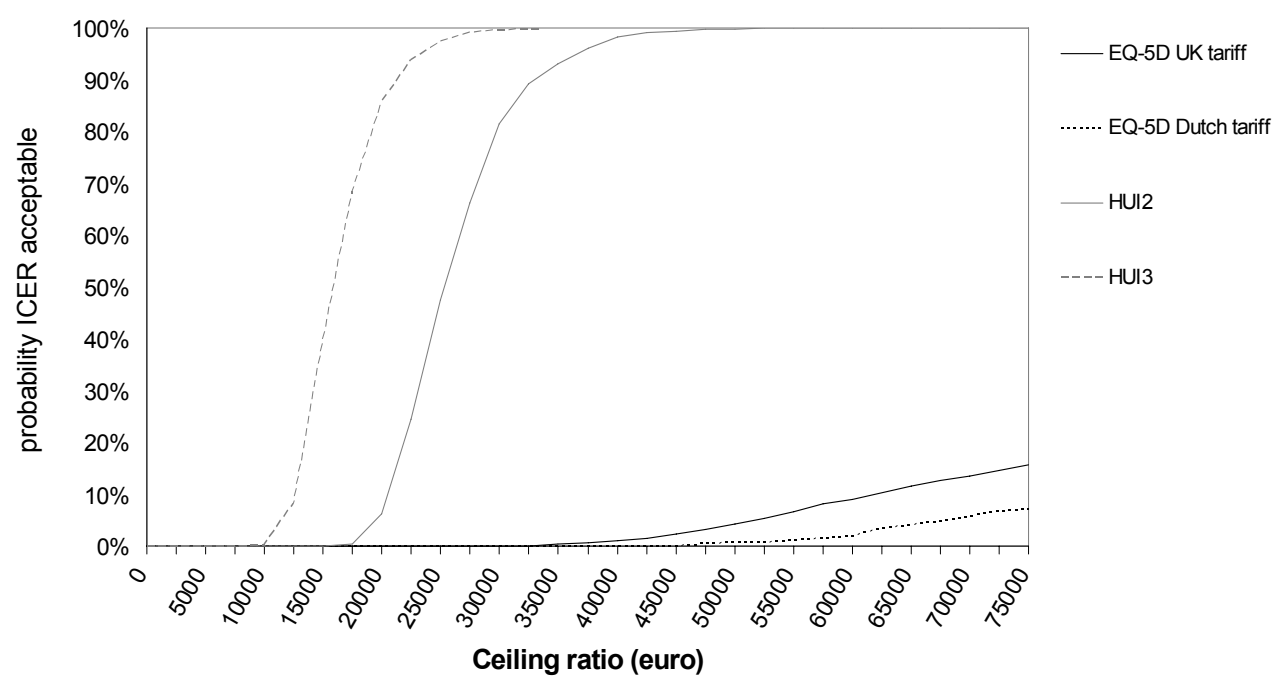

Figure 3.5 Cost-effectiveness acceptability curves of EQ-5D UK and Dutch tariff, HUI2 and HUI3 


\section{Conclusion and discussion}

Hearing loss affects the exchange of information and therefore affects a person's quality of life (Dalton et al., 2003). Hearing impaired persons can benefit from using a hearing aid, since hearing aid use has proven to be effective, it improves social, emotional and communication functions and reduces depression (Appollonio et al., 1996; Mulrow et al., 1990).

The results of this study in a relatively healthy population with hearing complaints provide insight in the differences between two widely used utility measures, the EQ-5D and the HUI system.

Regarding practicality, both questionnaires had high completion rates, with the EQ-5D having a higher completion rate than the HUI.

With the EQ-5D, differences were detected in utility by age and sex, indicating construct validity. The HUI3 detected differences by age, but not by sex. Differences between clinically distinctive groups were only detected by HUI3. However, the HUI3 did not confirm our expectation that non-applicants would have higher utility values than first-time applicants. An explanation for this may be that non-applicants had more health problems other than their hearing, as reflected in the ambulation and pain dimensions of the HUI3.

Overall, HUI2 and HUI3 scores were lower than EQ-5D scores and agreement was moderate to poor. Although these measures intend to assess the same construct, namely health state utility, this result was expected as the instruments differ in their underlying assumptions about what constitutes health state utility.

As to responsiveness, only HUI2 and HUI3 measured statistically significant improvement after hearing aid fitting, the EQ-5D UK and Dutch tariff both were not able to capture this effect. Half of the patients (53\%) who were fitted with a hearing aid were lost to follow up, either because they had not finished their hearing aid fitting before the end of the study, or because they had their hearing aid fitted at a dispenser not participating in our study. As these patients did not differ from the follow-up group in baseline utility, hearing loss and age, we did not expect this low response rate to influence the results.

The HUI2 and HUI3 change scores resulted in smaller ICERs for hearing aid fitting. Although they were only illustrative, the different ICERs found in the present study clearly show that the choice of a utility instrument in the economic evaluation of hearing aid fitting may heavily influence the cost-effectiveness outcome.

To calculate the ICER of hearing aid fitting we included the total population of respondents considered for hearing aid fitting, regardless of the type of hearing aid fitting. This makes the ICERs found in the present study representative for hearing aid fitting in general in the Netherlands. However, the one year ICERs calculated in the present study were merely illustrative of the impact of different utility scores on the ICER of hearing aid fitting, as the cost-effectiveness of hearing aid fitting has thoroughly been examined by Joore et al. (2003b).

Our results confirm Barton et al. (2005; 2004), who compared the EQ-5D UK tariff and HUI3 before and after hearing aid fitting in a UK sample. However, the change in utility after hearing aid fitting derived with the HUI3 in the present study was twice the change in utility derived with the HUI3 in the study of Barton et al. (2004). Respondents in the latter 
study had a lower baseline utility score and a higher range in utility change after hearing aid fitting, which suggests a somewhat different study population. Our results confirm previous studies that concluded that the EQ-5D lacks sufficient sensitivity to measure the benefit of hearing aid fitting (Bess, 2000; Joore et al., 2003a; Vuorialho et al., 2006b). The lower utility scores and higher responsiveness of the HUI3 in relation to the EQ-5D were also found in a population with visual impairment (Espallargues et al., 2005). Joore et al. (2003b) calculated that hearing aid fitting costs $€ 15807$ per QALY, using the EQ-5D as utility measure. The results of the present study that using the HUI3 as utility measure probably had resulted in a more favourable ICER for hearing aid fitting. Vuorialho et al. (2006a) recently concluded that counseling of hearing aid users to reduce the number of non-users is highly cost-effective, although they were unable to measure any change in utility. As they used the EQ-5D, it is possible that they would have been able to demonstrate favourable costs per QALY when they would have used the HUI3 to measure change in utility.

Three questions arise from the results of the present study: can differences be explained by differences in the measures, are the differences observed between the measures important, and what are the implications of the findings for utility measurement and cost-utility analysis in populations with hearing complaints?

First, differences in utility scores can be explained by differences in the descriptive system and the way the utility scoring function is derived. Regarding the descriptive system, the focus of the EQ-5D on physical, mental and social functioning (Brooks, 1996) differs from the 'within-the-skin' perspective of HUI, which focuses on the underlying level of impairment. However, this does not explain why the EQ-5D does not measure change after hearing aid fitting, as previous studies have found that hearing aid fitting improves social and emotional functioning (Appollonio et al., 1996; Mulrow et al., 1990). Also, the measures differ in the content and number of attributes, items and levels used, and therefore differ both in the number and in the content of possible health states. It has already been suggested not to use the EQ-5D in relatively healthy populations, given the presence of a ceiling effect (Kopec \& Willison, 2003). The ceiling effect of the EQ-5D found in the present study is likely to contribute to the differences in responsiveness. When, as found in our study, $44 \%$ of the respondents report perfect health at baseline, it is unlikely to find a considerable utility gain from any intervention. Furthermore, since the HUI descriptive system pays explicit attention to hearing abilities, it is to be expected that in a relatively healthy population with hearing complaints HUI and EQ-5D utility scores differ, and HUI is more responsive. As the HUI3 also pays explicit attention to visual abilities, this may explain why comparisons of EQ-5D and HUI3 in hearing and vision show similar results (Espallargues et al., 2005).

There are also differences in the utility scoring functions. Although in general SG (used for HUI) leads to higher scores than TTO (used for EQ-5D) (Drummond et al., 2005), in the present study the EQ-5D scores were considerably higher than the HUI scores. Although different populations do not necessarily yield different results (Drummond et al., 2005), the population sample in which the preferences are measured may also have impact on the differences. In the present study, differences between utilities derived with the UK and 
Dutch tariff were observed. This difference may be the result of differences in health valuation between people from the Netherlands and the UK, but may also result from the somewhat questionable representativeness of the population sample used to develop the Dutch tariff (Lamers et al., 2005).

Differences also exist in the type of scoring function. EQ-5D uses an additive system, assuming no interaction for preferences among attributes at all. The HUI uses a multiplicative scoring function, with the effect that the loss of utility associated with a particular dimension is dependent on the level of impairment on other dimensions (Drummond et al., 2005). For example, Barton et al. (2005) illustrated that hearing impairment ('unable to hear at all') has a greater impact on HRQoL as measured with the HUI3 when one has no other health problems $(-0.53)$, than when one also has moderate to severe pain and is unable to see at all $(-0.05)$. It seems rational that persons find their hearing loss a less important aspect of their health state utility when they experience more comorbidity. The multiplicative scoring function of HUI takes this influence of comorbidity into account and seems to be more suitable for modeling utility scores.

Are the differences observed between the measures important? The answer to this question is a clear 'yes'. The impact of different utility measures on the ICER for hearing aid fitting is of a magnitude that can alter policy decisions and emphasizes that comparisons of QALYs across studies and interventions should be interpreted with caution (Conner-Spady \& Suarez-Almazor, 2003). The general purpose of a utility measure is to capture the health effects in terms of HRQoL of a policy or program for use in economic analyses. Health economic analyses are a tool to allocate resources in a way that maximizes health (or welfare). In order to use the outcomes of economic analyses for policy decisions, there should however be a clear notion of what should be maximized. The results of the present study show that the potential benefit of an intervention heavily depends on the assumptions of what constitutes health underlying the utility measure that is used. Beyond the apparent necessity of psychometric quality of a utility measure, an important area for future research is whether societies wish, or should, maximize life expectancy corrected for HRQoL from a more functional perspective as in the EQ-5D, or from a 'within-the-skin' perspective as in the HUI. Other studies that have thoroughly examined the psychometric differences between utility measures seem to pass over this important question (Brazier et al., 2004; Feeny et al., 2004; O'Brien et al., 2003; Petrou \& Hockley, 2005; Pickard et al., 2005).

What are the implications of the findings for utility measurement and cost-utility analysis in populations with hearing complaints? Generally, it has been recommended that the instrument that is most sensitive to the health states in which one is interested should be selected (Conner-Spady \& Suarez-Almazor, 2003; Drummond et al., 2005; Guyatt et al., 1996). From clinical experience it is plain that hearing aid use is effective in alleviating hearing loss and does improve health-related quality of life, but the EQ-5D lacks the sensitivity to capture this improvement. In an otherwise healthy population, HUI3 has proven to be more responsive and therefore more appropriate for evaluating HRQoL in a population with hearing complaints, and is therefore the instrument of first choice in this population. 


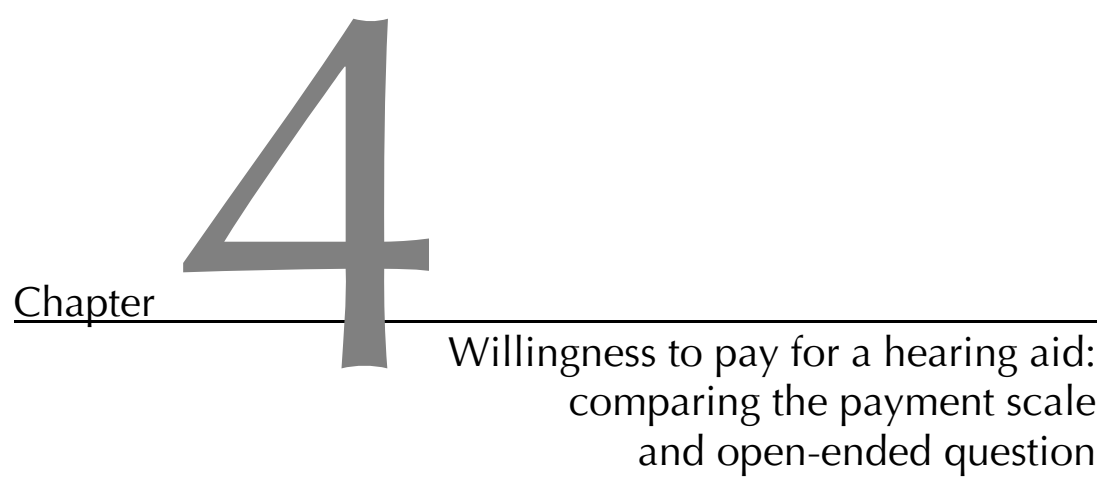

Janneke PC Grutters Lucien JC Anteunis Michelene N Chenault Manuela A Joore

Journal of Evaluation in Clinical Practice, accepted for publication 


\section{Abstract}

\section{Rationale \& objectives}

Different question formats elicit different willingness to pay (WTP) results, but there is no consensus on which method elicits the most valid WTP. In spite of the methodological controversies, WTP is a potentially valuable tool in health economics to value health services. Our general objective was to provide additional evidence on the validity of two WTP elicitation formats: the open-ended question and the payment scale.

\section{Method}

We elicited WTP for a hearing aid among hearing aid users $(n=108)$, using both a payment scale and an open-ended question. We compared the results from both formats. We tested criterion validity by comparing both formats with the actual out-of-pocket payment. Construct validity was tested by examining whether WTP was consistent with positive income elasticity.

\section{Results}

The WTP results elicited with the payment scale and open-ended question were not statistically significantly different. Both formats showed good criterion validity, although the open-ended question showed a stronger association with the actual out-of-pocket payment. The open-ended format showed better construct validity, as it was influenced by family income.

\section{Conclusion}

The results of the present study showed that the open-ended question was more valid than the payment scale question. We therefore recommend that in future WTP studies on hearing aids the open-ended question is used to directly elicit WTP values. The same recommendation may apply to other studies where respondents are familiar with costs or payments for the intervention under evaluation. 


\section{Background}

In the last decade there has been renewed interest in cost-benefit analysis (CBA) as a method for the economic evaluation of health care interventions (Diener et al., 1998). CBA distinguishes itself from cost-effectiveness analysis in that not only the costs, but also the benefits of health care interventions are valued in monetary units (Drummond et al., 2005). A common method to estimate monetary values for health care interventions is direct willingness to pay (WTP), also known as contingent valuation. In the WTP approach, the maximum amount that an individual is willing to pay to obtain a good is elicited using a hypothetical scenario by means of a survey. The WTP technique was originally developed by environmental economists, but is increasingly used in health economics. Despite its widespread use, there are some methodological controversies, for example with respect to the type of question asked (Diener et al., 1998; O'Brien \& Gafni, 1996). There are four types of questions to directly measure maximum WTP: the open-ended, payment scale, bidding game and discrete-choice format (Frew et al., 2003; O'Brien \& Gafni, 1996; Ryan et al., 1997; Smith, 2000). In an open-ended question the respondent is asked to state the maximum amount of money he or she is willing to pay to receive a certain intervention, without any clues or options given. In the payment scale approach, each respondent chooses an amount from the same pre-specified and ordered list of WTP amounts. When using the bidding game, an interviewer suggests WTP amounts that the respondent accepts or rejects and continues to make higher or lower offers depending on whether the respondent accepts or rejects the previous offers. In the discrete-choice format, different respondents receive different offers, and each respondent is required to make an accept/reject choice at this single, predetermined offer. As the bidding game format requires face-to-face interviews and the discrete choice format requires a very large number of respondents, both are not feasible in all situations (Klose, 1999). Different question formats have been found to elicit different WTP results, but there is no consensus on which method elicits the most valid WTP (Diener et al., 1998; O'Brien \& Gafni, 1996; Onwujekwe, 2004; Smith, 2000). In spite of the methodological controversies, WTP is a potentially valuable tool in health economics to value health services (Olsen \& Donaldson, 1998). The present study therefore provides additional evidence on the validity of two elicitation formats: the open-ended question and the payment scale. We collected this evidence in a study on willingness to pay for hearing aids. Our first objective was to examine whether an open-ended question and payment scale question elicit different monetary values. Our second objective was to explore which of the two elicitation formats is more valid. 


\section{Methods}

\section{Setting}

Hearing loss is one of the most common forms of sensory impairment, with an estimated prevalence of $15 \%$ in the western adult population (Davis, 1995; Johansson \& Arlinger, 2003). Hearing aid fitting has been proven to be effective (Larson et al., 2000). Nevertheless, in the past decade, health care policy makers have begun to demand economic evidence regarding the efficiency of hearing aids. Joore et al (2003b) demonstrated that hearing aid fitting is a cost-effective health care intervention. In light of the renewed interest in $\mathrm{CBA}$ as a method for the economic evaluation of health care interventions a study was carried out to examine how much hearing aid users are willing to pay for a hearing aid.

In most countries hearing aid users have to pay for their hearing aids. In the Netherlands, patients with a hearing loss exceeding $35 \mathrm{~dB}$, averaged over 1,2 and $4 \mathrm{kHz}$ in the better ear, are entitled to a maximum reimbursement by their medical insurance of $€ 657,50$ per hearing aid. This reimbursement is doubled when patients are fitted with a hearing aid on both ears (binaural fitting). Generally, the hearing aid dispenser collects the reimbursement and the patient is billed only for that part of the hearing aid price (including the costs of fitting) that exceeds the reimbursement. The latter will be referred to as the out-of-pocket payment. In contrast to most WTP studies in health care, the amount a person is willing to pay can be compared to what the person actually has paid for a hearing aid. This makes hearing aid provision a useful subject to examine the validity of WTP.

\section{Study design and population}

The study design was a survey. A questionnaire, designed for self-completion without supervision, was distributed at three hearing aid dispenser practices and two Audiological Centres in and near Maastricht, the Netherlands. The study population consisted of adult hearing aid users, both first time and experienced hearing aid users. For the duration of one week in 2003 all consulting hearing aid users were asked to fill out this questionnaire while attending the practice or centre.

\section{Questionnaire}

The total questionnaire consisted of 40 questions regarding quality of life, hearing handicap, demographic items as well as items addressing the maximum WTP for a hearing aid and the amount the respondent had actually paid for his or her hearing aid. The present analysis focused on the WTP questions and the actual out-of-pocket payment question. Both WTP and actual out-of-pocket payment were defined as the amount exceeding the reimbursement, since in the Netherlands hearing aid users are only billed for this part of the hearing aid price. We elicited WTP with the two formats most applicable for use in a questionnaire for self-completion: the payment scale and open-ended format. In question 
26 the respondents were asked to state the maximum amount they were willing to pay outof-pocket for one hearing aid (Appendix 4.1) on a payment scale. The payment scale had the following categories: not willing to pay, € 50, € 100, € 250, €500, € 1000 and $€ 2000$. The range was based on the actual range of out-of-pocket payments for a hearing aid in the Netherlands. At the end of the questionnaire (question 40) the exact same WTP question was asked, now using the open-ended format (Appendix 4.1). The actual out-of-pocket payment for the respondent's current hearing aid(s) was elicited using an open-ended format (question 11; Appendix 4.1). The questionnaire also included several demographic questions regarding age, sex, marital status, education, and family income. Respondents were requested to answer all questions. The complete questionnaire in Dutch is available upon request.

\section{Data analysis}

Descriptive statistics were obtained for the demographics, the payment scale and the openended WTP question. The results on the payment scale WTP question were coded as the amounts corresponding to the payment scale categories (not willing to pay, $€ 50, € 100$, $€ 250$, $€ 500$, $€ 1000$ and $€ 2000$ ). Normality of the data was tested using the KolmogorovSmirnov test. A Wilcoxon Signed Ranks Test was used to test whether the open-ended and payment scale format elicited different WTP, and Spearman correlation was calculated to examine whether and how strongly they were related. In conformance with Cohen (1988), correlation coefficients smaller than 0.3 were considered weak, between 0.3 and 0.5 moderate and higher than 0.5 a strong relationship. Also, the results on both the payment scale and open-ended WTP question were analyzed using multiple regression analysis to assess whether the outcome on one WTP question influenced the outcome on the other WTP question. The WTP values were used as the dependent variables. In case the dependent variables were not normally distributed, we examined the skewness and kurtosis and, if necessary, transformed the values by taking the square root to obtain acceptable distributions. In both analyses the other WTP question was considered as possible explanatory variable.

To explore which format elicited more valid results, we tested the criterion and construct validity of both the payment scale and open-ended WTP question. The criterion validity can be examined by comparing the results with a gold standard (Streiner \& Norman, 1995). For WTP questions, actual payments are the gold standard (Drummond et al., 2005; Onwujekwe, 2004). We therefore compared the WTP results with the actual out-of-pocket payment to examine the criterion validity. In cases where a respondent was binaurally fitted, the answer on the out-of-pocket payment question was divided by two, to obtain the actual out-of-pocket payment per hearing aid. A Wilcoxon Signed Ranks Test was used to test whether WTP and actual out-of-pocket payment were different, and Spearman correlation was calculated to examine whether and how strongly they were related. Also, we considered actual out-of-pocket payment as explanatory variable in the multiple regression analysis, to examine whether actual out-of-pocket payment was associated with WTP. 
The construct validity can be examined by determining whether the results are consistent with theoretical constructs (Streiner \& Norman, 1995). We tested whether the data were consistent with a theoretical construct from economic theory: positive income elasticity (Maxwell, 1981), meaning that higher incomes should be associated with higher WTP. First, Spearman correlation was calculated to examine whether and how strongly family income and WTP were related. Next, we considered family income as explanatory variable in the regression analysis.

Besides the other WTP question, the actual out-of-pocket payment and family income, age and hearing aid experience were included as explanatory variables in the regression models. We expected younger respondents to state a higher WTP value, since it is more likely that they need hearing aids for work or social activities. We expected persons with hearing aid experience to state higher WTP values since they are likely to attach more value to, or be more aware of, the benefits of hearing aids. Pairwise inclusion was used, meaning that cases which had a valid value for at least one of the independent variables were included. All models were fitted by backward elimination and only the final models are reported in this paper.

For all tests a p-value smaller than 0.05 was considered to be statistically significant. All analyses were performed using the Statistical Package for the Social Sciences, version 12.01 .

\section{Results}

\section{Study population}

A total of 108 respondents completed the questionnaire (Table 4.1). The majority of the respondents were male (57\%) and the mean age was 67.8 years (standard deviation 12.4). Sixteen percent reported a monthly family income of less than $€ 1150$ and $7 \%$ a family income above $€ 3400$. Approximately half of the respondents $(48 \%)$ were first time hearing aid users and $45 \%$ were experienced hearing aid users, for $7 \%$ hearing aid experience was unknown. Of the respondents $29 \%$ were monaurally and $57 \%$ were binaurally fitted. Almost all respondents (93\%) stated to be satisfied with their hearing aid.

\section{Payment scale versus open-ended format}

For the total study population, maximum willingness to pay for a hearing aid was on average $€ 329$ (sd 445) when measured with the payment scale and $€ 316$ (sd 337) when measured with the open-ended question (Table 4.2). Median scores were $€ 250$ for both WTP questions. The data were not normally distributed (Kolmogorov-Smirnov, p-values < 0.01), but were skewed to the right. Therefore, non-parametric statistics were used. However, as mean WTP results are used in economic analyses and therefore are highly informative, we also list means, standard deviations and ranges. 
Table 4.1 Characteristics of the study population. Values are numbers (percentages) unless stated otherwise

\section{Characteristics}

Number of respondents

Age

Mean (standard deviation)

$67.8(12.4)$

Range

$27.9-92.5$

Male

Marital status

Married

Widow/ widower

Not married

Living together

Missing

Education

Primary school

Lower education

Junior general secondary education (MAVO)

Senior secondary vocational education (MBO)

Senior general secondary education (HAVO/VWO)

Higher professional education (HBO) / university

Missing

Family income

$$
\begin{aligned}
& € 400-1150 \\
& € 1150-1600 \\
& € 1600-2500 \\
& € 2500-5000 \\
& \text { Don't know }
\end{aligned}
$$

Not willing to answer

Missing

Hearing aid experience

First time hearing aid user

Experienced hearing aid user

Missing

Type of hearing aid fitting

$$
\begin{aligned}
& \text { Monaural } \\
& \text { Binaural } \\
& \text { Missing }
\end{aligned}
$$

$\begin{array}{rr}73 & 67.6 \% \\ 21 & 19.4 \% \\ 9 & 8.3 \% \\ 2 & 1.9 \% \\ 3 & 2.8 \%\end{array}$

$21 \quad 19.4 \%$

$22 \quad 20.4 \%$

$25 \quad 23.1 \%$

$13 \quad 12.0 \%$

$9 \quad 8.3 \%$

$14 \quad 13.0 \%$

$4 \quad 3.7 \%$

$17 \quad 15.7 \%$

$15 \quad 13.9 \%$

$16 \quad 14.8 \%$

$11 \quad 10.1 \%$

$9 \quad 8.3 \%$

$21 \quad 19.4 \%$

$19 \quad 17.6 \%$

$52 \quad 48.1 \%$

$49 \quad 45.4 \%$

$7 \quad 6.5 \%$

$31 \quad 28.7 \%$

$61 \quad 56.5 \%$

$16 \quad 14.8 \%$

$97 \quad 93.3 \%$ 
Table 4.2 Descriptives and comparison of the results of the payment scale and the open-ended WTP question

\begin{tabular}{|c|c|c|c|c|c|c|c|c|}
\hline Total group & $\begin{array}{r}\text { Valid N } \\
\text { total } \\
\end{array}$ & $\begin{array}{r}\text { Missing } \\
\mathrm{N}(\%) \\
\end{array}$ & $\begin{array}{r}\text { Mean (sd) } \\
€\end{array}$ & Range & $\begin{array}{r}\text { Median } \\
€ \\
\end{array}$ & $\begin{array}{r}\text { IR } \\
€\end{array}$ & p-value ${ }^{1}$ & $\begin{array}{c}\text { Spearman } \\
\text { correlation }\end{array}$ \\
\hline Payment scale WTP & 99 & 9 (8) & $329(445)$ & $0-2000$ & 250 & 400 & & \\
\hline Open-ended WTP & 70 & $38(35)$ & $316(337)$ & $0-1500$ & 250 & 325 & & \\
\hline \multicolumn{9}{|l|}{ Group for comparison } \\
\hline Payment scale WTP & 67 & $41(38)$ & $323(382)$ & $0-2000$ & 250 & 400 & \multirow{2}{*}{0.879} & \multirow{2}{*}{$0.775^{* *}$} \\
\hline Open-ended WTP & 67 & $41(38)$ & $320(341)$ & $0-1500$ & 250 & 300 & & \\
\hline
\end{tabular}

Abbreviations: IR = interquartile range; sd = standard deviation; WTP = willingness to pay

${ }^{1}$ Wilcoxon Signed Ranks Test

** Correlation is significant at the 0.01 level (2-tailed)

The 67 respondents who answered both the payment scale and open-ended WTP question had mean WTP values of $€ 323$ (sd 382) and $€ 320$ (sd 341) respectively (Table 4.2). The median scores on both the open-ended and payment scale WTP question were $€ 250$ and showed no statistically significant difference (Wilcoxon Signed Ranks Test, p-value .879). The results on the payment scale WTP question ranged from not willing to pay to $€ 2000$, and on the open-ended WTP question the results ranged from not willing to pay to $€ 1500$. There were statistically significantly more missing values on the open-ended WTP question (35\%) than on the payment scale WTP question (8\%). The WTP questions had a Spearman Correlation of .775 (p-value .000), indicating a statistically significant and strong relationship.

The regression analyses showed that the results on both WTP formats were positively and statistically significantly associated with each other (Table 4.3).

Table 4.3 Final models of the multiple regression analysis for both the payment scale and openended WTP question

\begin{tabular}{lccccccr}
\hline & \multicolumn{3}{c}{ Payment scale WTP question } & \multicolumn{3}{c}{$\begin{array}{c}\text { Open-ended WTP question } \\
\text { (square root transformation) }\end{array}$} \\
\cline { 2 - 8 } & \multicolumn{3}{c}{ Regression model } & \multicolumn{4}{c}{ Regression model } \\
\cline { 2 - 8 } Independent variable & $\mathbf{b}$ & S.E. & p-value & b & S.E. & p-value \\
\hline Constant & -1.63 & 0.63 & 0.012 & 6.79 & 4.63 & 0.151 \\
Open-ended WTP question & 0.13 & 0.012 & 0.000 & & & \\
$\begin{array}{l}\text { Payment scale WTP question } \\
\text { Age }\end{array}$ & & & & 4.42 & 0.54 & 0.000 \\
Family income & 0.03 & 0.008 & 0.000 & -0.13 & 0.06 & 0.041 \\
Actual out-of-pocket payment & & & & 1.13 & 0.43 & 0.012 \\
\hline & & & & 0.01 & 0.00 & 0.026 \\
\hline
\end{tabular}

Abbreviations: WTP = willingness to pay; S.E. = standard error 


\section{Validity}

To test the criterion validity, we compared the WTP results with the actual out-of-pocket payments. Of the 108 respondents, 60 answered both the payment scale WTP question and the actual out-of-pocket payment question (Table 4.4). Thirty respondents (50\%) stated a higher WTP than their actual out-of-pocket payment, while for 28 respondents (47\%) WTP was lower than their actual out-of-pocket payment. The payment scale WTP question and actual out-of-pocket payment had median scores of $€ 250$ and $€ 339$ respectively and were not statistically significantly different (Wilcoxon Signed Ranks Test, p-value .301). The payment scale WTP question and actual out-of-pocket payment had a Spearman Correlation of .277 ( $p$-value .032), indicating a statistically significant, weak relationship. The regression analysis indicated that the actual out-of-pocket payment did not significantly influence the results on the payment scale WTP question (Table 4.3).

Answers on both the open-ended WTP question and the actual out-of-pocket payment question were provided by a total of 47 respondents (Table 4.4). Of them, 21 (45\%) had a higher WTP than actual out-of-pocket payment, and 25 (53\%) had a lower maximum WTP than actual out-of-pocket payment. The median scores of the open-ended WTP question and the actual out-of-pocket payment were $€ 250$ and $€ 277$ respectively and were not significantly different (Wilcoxon Signed Ranks Test, p-value .260). The results on the openended WTP question and the actual out-of-pocket payment had a Spearman Correlation of .383 (p-value .008), indicating a statistically significant, moderate relationship. The regression analysis showed that the actual out-of-pocket payment significantly and positively influenced the results on the open-ended WTP question (Table 4.3).

To test for construct validity, we examined whether higher family income was associated with higher WTP. The results on the payment scale WTP question and family income had a Spearman Correlation of .209 (p-value .130), indicating no relationship. In the regression analysis with the payment scale WTP question as dependent variable, family income was not associated with WTP.

Table 4.4 Comparison of the actual out-of-pocket payments with the payment scale and openended WTP question

\begin{tabular}{lrrrrrrrr}
\hline & $\begin{array}{l}\text { Valid N } \\
\text { listwise }\end{array}$ & $\begin{array}{rrrrr}\text { Missing } \\
\mathbf{N}(\%)\end{array}$ & $\begin{array}{r}\text { Mean (sd) } \\
\boldsymbol{\epsilon}\end{array}$ & $\begin{array}{r}\text { Range } \\
\boldsymbol{\epsilon}\end{array}$ & $\begin{array}{r}\text { Median } \\
\boldsymbol{\epsilon}\end{array}$ & IR & p-value $^{1}$ & $\begin{array}{r}\text { Spearman } \\
\text { correlation }\end{array}$ \\
\hline Actual payment & 60 & $48(44)$ & $398(277)$ & $0-1000$ & 339 & 433 & 0.301 & $0.277^{*}$ \\
Payment scale WTP & 60 & $48(44)$ & $398(531)$ & $0-2000$ & 250 & 400 & & \\
& & & & & & & & \\
Actual payment & 47 & $61(57)$ & $354(251)$ & $0-1000$ & 277 & 351 & 0.260 & $0.383^{* *}$ \\
Open-ended WTP & 47 & $61(57)$ & $307(340)$ & $0-1500$ & 250 & 300 & & \\
\hline
\end{tabular}

Abbreviations: $s d=$ standard deviation; $I R=$ interquartile range; $W T P=$ willingness to pay

${ }^{1}$ Wilcoxon Signed Ranks Test

* Correlation is significant at the 0.05 level (2-tailed)

** Correlation is significant at the 0.01 level (2-tailed) 
The results on the open-ended WTP question and family income had a Spearman Correlation of .427 (p-value .004), indicating a statistically significant, but moderate relationship. With the open-ended question as dependent variable, regression analysis showed that a higher WTP was associated with a higher income (Table 4.3).

Lower age was associated with higher WTP in the regression model with the open-ended WTP question as dependent variable (Table 4.3). However, in the regression model with the payment scale WTP question as dependent variable, higher age was associated with higher WTP. In both question formats, hearing aid experience did not influence WTP.

\section{Discussion}

The present study examined willingness to pay for a hearing aid and adds to the limited evidence available on the validity of direct willingness to pay formats.

On average, hearing aid users in the Netherlands were willing to pay at maximum $€ 329$ for a hearing aid when measured with the payment scale WTP question, and $€ 316$ when measured with the open-ended WTP question. We found no statistically significant difference, and a strong association between both formats. Thus, in a population of hearing aid users, who are familiar with payment for their hearing aid, the payment scale and openended question do not result in statistically significantly different results. Previous studies on other health care interventions did report an influence of elicitation methods on WTP (Donaldson et al., 1997; Frew et al., 2003; Klose, 1999; Whynes et al., 2003). The realistic setting and high level of understanding and experience of the respondents with hearing aids in our study may have led to more stable answers (Onwujekwe et al., 2005). The percentage missing values in the present study was much higher for the open-ended question (35\%) than for the payment scale question (8\%). The open-ended question seems more difficult to answer, possibly because no cues are provided. The payment scale question is generally considered to be more valid then the open-ended question, because the open-ended question is thought to be too hypothetical and provides insufficient cues (Arrow et al., 1993; Donaldson et al., 1997; Ryan et al., 2004). However, the payment scale question is also known to cause range bias (Whynes et al., 2004). It may be so that responses on the open-ended question are more valid because no cues are provided, as no range bias or starting point bias will occur. Also, only persons who have thoroughly considered their WTP and are able to construct a WTP value 'on their own' answer the open-ended question (Cookson, 2003). This may lead to less, but more valid answers. The latter is supported by the validity tests we performed. Both the payment scale format and the open-ended format showed good criterion validity, but the open-ended WTP question showed a stronger association with the actual out-of-pocket payment. Moreover, the openended WTP question showed better construct validity, since it was consistent with the theoretical construct of positive income elasticity. Also, the open-ended format confirmed our expectation that younger respondents were willing to pay more for a hearing aid.

There are some limitations of the present study that need to be addressed. First, due to the setting no information was available regarding primary non-response. Little is known about 
response patterns in persons with hearing complaints attending audiological centres and hearing aid dispenser practices. In a study in a similar setting, a non-response of $25 \%$ out of 402 persons asked has been found (unpublished data, 2007). Probably, the present study had an even smaller percentage of non-response, since the questionnaire was less timeconsuming and easier to complete. Also, there was a large proportion of missing values on the actual out-of-pocket payment question (42\%). This can be explained by the fact that some respondents were still in the process of their first hearing aid fitting, and had therefore not made an out-of-pocket payment yet (29\% of the missing values) or that we did not know whether they had a monaural or binaural fitting (20\% of the missing values). Missing values may have also been a result of persons having difficulty remembering what they had paid for their hearing aid. Furthermore, the fact that all respondents answered the payment scale question before the open-ended question may have caused an ordering effect. This would bias the results of the comparison between both question formats. However, by dispersing the WTP questions as much as possible over the questionnaire we have attempted to minimize an ordering effect.

\section{Conclusion}

The present study adds to the limited evidence on the validity of direct willingness to pay measures. Although the open-ended format is thought to be less valid than the payment scale format, the results of the present study point towards the opposite. We therefore recommend that in future WTP studies on hearing aids the open-ended question is used to directly elicit WTP values. The same recommendation may apply to other studies where respondents are familiar with costs or payments for the intervention under evaluation. 


\section{Appendix 4.1}

Actual out-of-pocket payment question:

11. How much have you paid out-of-pocket for your current hearing aid(s)?

o $€$

Payment scale WTP question:

26. How much are you, at a maximum, willing to pay out-of-pocket for one new hearing aid?
o $€ 50$
o $€ 100$
o $€ 250$
o $€ 500$
o $€ 1000$
o $€ 2000$

o I am not willing to pay out-of-pocket for a new hearing aid

Open-ended WTP question:

40. How much are you, at a maximum, willing to pay out-of-pocket for one new hearing aid?

o $€$ 


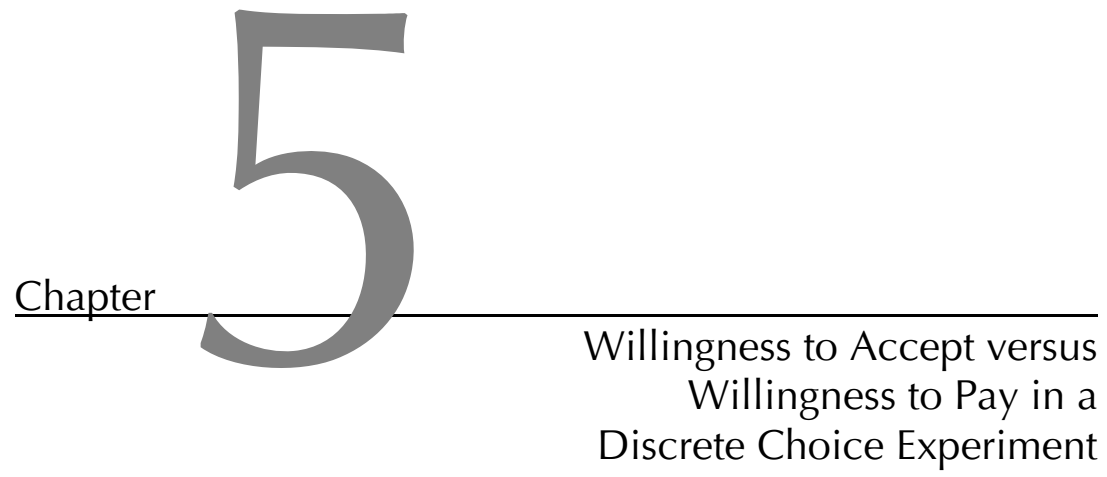

Janneke PC Grutters Alfons GH Kessels Carmen D Dirksen Debby van Helvoort-Postulart Lucien JC Anteunis Manuela A Joore 


\section{Abstract}

\section{Objectives}

Our main objective was to compare willingness to accept (WTA) and willingness to pay (WTP) in a discrete choice experiment on hearing aid provision. Additionally, income effect and endowment effect were explored as possible explanations for the disparity between WTA and WTP, and the impact of using a WTA and/or WTP format to elicit monetary valuations on the net benefit of the new organization of hearing aid provision was examined.

\section{Methods}

Choice sets were based on five attributes: performer of the initial assessment, accuracy of the initial assessment, duration of the pathway, follow-up at the Ear Nose and Throat (ENT) specialist, and costs. Persons with hearing complaints randomly received a WTP (cost attribute defined as extra payment) or WTA (cost attribute defined as discount) version of the experiment. In the versions, except for the cost attribute, all choice sets were equal.

\section{Results}

The cost coefficient was statistically significantly higher in the WTP format. Marginal WTA was statistically significantly higher than marginal WTP for the attributes accuracy and follow-up. Disparity was higher in the high educational (as proxy for income) group. We did not find proof of an experience endowment effect. Implementing the new intervention would only be recommended when using WTP.

\section{Conclusions}

WTA exceeds WTP, also in a discrete choice experiment. As this affects monetary valuations, more research on when to use a payment or a discount in the cost attribute is needed before discrete choice results can be used in cost-benefit analyses. 


\section{Introduction}

Studies that directly measure monetary values have often found a disparity between a person's willingness to pay for a good and his willingness to accept compensation to forgo the same good (Horowitz \& McConnell, 2002; O'Brien et al., 2002). The aim of our study was to examine whether this disparity also occurs when one indirectly measures monetary values in a discrete choice experiment.

Monetary valuations are often elicited using contingent valuation (Bayoumi, 2004; Diener et al., 1998). In contingent valuation studies one directly asks respondents how much money they would be willing to pay or how much compensation they would request for a hypothetical intervention. The resulting willingness to pay (WTP) or willingness to accept (WTA) is a measure of how much the respondent values the intervention. Following Hicks (1943), one can use either the concept of compensating variation or the concept of equivalent variation to measure monetary values. Compensating variation measures the amount of money that is required after the change to make a respondent's level of utility the same as before the change, while equivalent variation measures the amount of money that is required before the change, to make utility the same as it would be after the change. Within both these concepts a distinction can be made between WTA (when a compensation is required) and WTP (when a payment is required).

Numerous contingent valuation studies have found that WTA exceeds WTP (Horowitz \& McConnell, 2002; O'Brien et al., 2002), but in health care this disparity has only been examined four times (Borisova \& Goodman, 2003; Carthy et al., 1999; O'Brien et al., 1998; Van den Berg et al., 2005b). Several explanations for the disparity between WTA and WTP have been suggested (Brown \& Gregory, 1999). Standard economic theory allows for two possible explanations, being an income effect and a lack of substitutes (Hanemann, 1991). An income effect would occur because payment is constrained by income, while demand of compensation is not. A total lack of substitutes would make it impossible to compensate an individual for the removal of the good, and would therefore lead to extreme WTA values. Another possible explanation, advanced by psychologists instead of economists, is the endowment effect (Kahneman \& Tversky, 1979). The endowment effect is closely related to loss aversion, and suggests that desirable goods are more valuable when they are part of one's endowment. That is, persons value loss of something they own or have experienced higher than acquiring the same thing when do not have or have experienced it. Other possible explanations are related to the difference between buyers and sellers, and are explained elsewhere (Brown \& Gregory, 1999).

In contingent valuation studies, a difference is made between persons who would gain utility from implementing the intervention (potential 'gainers') and persons who would lose utility from implementing the intervention (potential 'losers'). Potential gainers should be asked to state their maximum WTP for the intervention, while potential losers should be asked to state their minimum WTA for the intervention (Drummond et al., 2005; O'Brien \& Gafni, 1996). However, it has also been advocated to always use WTP, as WTP results are judged to be more valid and more conservative (Arrow et al., 1993; Bayoumi, 2004). 
An alternative method to elicit monetary valuations is discrete choice experimentation (Ryan, 2004; Ryan \& Gerard, 2003). Discrete choice experiments are developed in mathematical psychology and marketing, and are increasingly used in health services research (Louviere et al., 2000; Ryan \& Farrar, 2000; Ryan \& Gerard, 2003). When a cost attribute is included in the experiment, the willingness to pay or the willingness to accept compensation for a unit change in an attribute can be calculated. Subsequently, each alternative set of change in the levels of the attributes can be given a monetary value, which allows for use in a cost-benefit analysis, where the costs of a program are compared to its monetary benefits (Drummond et al., 2005). Much of the appeal of discrete choice experimentation lays in that it enables us to understand the trade-offs between attributes. This can for instance provide valuable information for health care workers deciding between alternative ways of the provision of a health care commodity. Another advantage of discrete choice experimentation is that it does not directly ask respondents to express an amount of money, so strategic behavior or protest answers are less likely to occur (Van den Berg et al., 2005a).

Until now, to our knowledge no publications have addressed the disparity between WTA and WTP in discrete choice experiments. As a result, lack of clarity exists on when to use WTA or WTP in discrete choice experimentation.

The main objective of our study was to examine whether using a WTA and a WTP format for the cost attribute in a discrete choice experiment elicits different preferences and monetary values. We examined this in a discrete choice experiment on transferring elements of hearing aid provision from the medical sector to private hearing aid dispensers. If we find a disparity between WTA and WTP, our second objective will be to explore whether two possible explanations for the disparity, being the income and endowment effect, influence the disparity between WTA and WTP in a discrete choice experiment. Our third objective will be to examine the effect of using a WTA format, a WTP format, and a WTA format for potential losers and a WTP format for potential gainers to elicit monetary values on the net benefit of the new organization of hearing aid provision.

\section{Methods}

\section{Setting}

Approximately $10 \%$ of the general population of western countries is hearing impaired, and this prevalence heavily increases with age (Chorus et al., 1995; Davis, 1995). For most hearing-impaired persons hearing aid fitting is an effective intervention (Larson et al., 2000; Mulrow et al., 1990). In the Netherlands, persons with a hearing loss exceeding $35 \mathrm{~dB}$, averaged over 1,2 and $4 \mathrm{kHz}$ in the better ear, are entitled to a reimbursement by their medical insurance. Generally, the hearing aid dispenser collects the reimbursement and the patient is billed only for that part of the hearing aid price that exceeds the reimbursement, which is referred to as the out-of-pocket payment. 
Given the ageing of the population the prevalence and, as a result, the health care costs associated with hearing rehabilitation will continue to increase. Because of the increasing costs and anticipated shortage of Ear Nose and Throat (ENT) specialists in the care for hearing-impaired persons, direct hearing aid provision by private hearing aid dispensers is currently under evaluation in the Netherlands, as well as in other countries in Europe. Besides issues of safety and efficiency, it is important to consider patient preferences regarding the organization of hearing aid provision. Direct hearing aid provision by private dispensers can be perceived as both favorable and unfavorable, depending on patients' individual preferences. In a previous study (Grutters et al., 2007b) we asked persons with hearing complaints to grade direct hearing aid provision and provision by referral. We found that $42 \%$ of the respondents preferred hearing aid provision by referral, $39 \%$ preferred direct hearing aid provision, and 18\% graded them equally. As there are patients who prefer direct hearing aid provision as well as patients who prefer provision by referral, implementation of direct hearing aid provision will generate both gainers and losers. This makes the case of direct hearing aid provision especially suitable for examining the disparity between WTA and WTP.

\section{Questionnaire design}

Twelve possible attributes of interest were identified by experts. Of these attributes, the five most important were identified by hearing-impaired persons $(n=21)$ in a preliminary survey, using five point Likert scales. These were 1) the performer of the initial assessment to distinguish between persons in need of medical care (referred to as patients) and persons not in need of medical care (clients), 2) the accuracy of the initial assessment, 3) the duration of the total hearing aid provision, 4) the follow-up at the ENT-specialist to evaluate the hearing aid, and 5) costs. Realistic levels, based on the results of a previous study (AZOS working group, 2006), were assigned to each attribute (Table 5.1). As we assumed the private dispenser to be equally or less accurate than the ENT-specialist, the attributes performer and accuracy of the initial assessment had levels that could not logically be varied independently from each other. Following Louviere et al. (2000), these attributes were combined. The cost attribute was assigned four levels, equal to two other attributes, to minimize an attribute effect. The range of the cost attribute was based on the answers on an open-ended question in the preliminary survey and the levels were based on actual Euro notes. 
Table 5.1 Attributes and levels used for discrete choice experiment

\begin{tabular}{ll}
\hline Attributes & Levels \\
\hline Performer and accuracy of initial assessment: & $*$ ENT-specialist at hospital \\
& $*$ Dispenser at practice, equally accurate \\
& $*$ Dispenser at practice, 10\% less accurate \\
& $*$ Dispenser at practice, 20\% less accurate \\
& $* 2$ months \\
& $* 4$ months \\
& $* 6$ months \\
& $* 8$ months \\
& $*$ Yes \\
& $*$ No \\
\hline Follow-up at ENT-specialist: & $*$ No \\
& $* € 20$ \\
\hline Discount for WTA / extra payment for WTP: & $* € 50$ \\
& $* € 100$ \\
\hline
\end{tabular}

We used a fractional factorial design to reduce the number of choices to the smallest number necessary for an efficient design. Using the SPSS orthoplan procedure we created an orthogonal main effects plan of the four attributes (three attributes with four levels, and one attribute with two levels), which ensured both level balance and orthogonality. Subsequently, 16 choice pairs were determined using the 'shifting' method, recommended by Street et al. (2005), ensuring level balance, minimal overlap and orthogonality. In the questionnaire respondents were asked to choose their preferred alternative from each choice pair, and we included the option that they found the alternatives equally attractive (no preference).

We created two versions of the questionnaire. In one version the cost attribute was defined as an extra payment to be paid by the participant (WTP format) on top of his out-of-pocket payment for the hearing aid. In the other version the cost attribute was defined as a discount off the out-of-pocket payment for the hearing aid (WTA format). By framing the cost attribute in relation to the out-of-pocket payment we tried to make it as realistic as possible. Respondents were explicitly told that when they had no out-of-pocket payment for their hearing aid, the discount would be handed to them in cash in the WTA format, or they still had to pay the extra payment in the WTP format. As the difference between the attribute levels of two alternatives in a choice set is used as explanatory variable in the analysis, for each choice set the increment in the cost attribute was equal in the two versions. This means that when in the WTP format the extra payment was $€ 20$ higher for alternative $B$ than for alternative $A$, in the WTA version the discount was $€ 20$ lower for alternative $B$ than for alternative $A$. This resulted in an increment of $€ 20$ in both formats. Apart from the cost attribute, all choice sets were equal in the WTA and WTP version of the questionnaire. An example of a discrete choice question is given in Appendix 5.1. 
Additionally we defined a choice set in which one alternative with a lower extra payment (or a higher discount), higher accuracy, and all other attribute levels equal unquestionably dominated the other, to check whether respondents understood the experiment. The total questionnaire therefore consisted of 17 discrete choice questions.

\section{Survey procedure}

In a pilot study, it was examined whether the participants found all attributes important and whether they were willing to trade between the attributes, to check whether the chosen attributes and levels were appropriate. It was also examined whether the formulation was correct, and whether the participants understood the experiment. The pilot questionnaire, completed by 35 participants, was administered face-to-face by a trained interviewer at a private dispenser practice. Based on the results of the pilot study, we slightly adjusted the range of the duration attribute. Following the pilot study, two trained interviewers conducted face-to-face interviews at four private hearing aid dispenser practices, chosen to be representative for the region. Participants were persons with hearing complaints visiting the dispenser. The WTA and WTP versions were randomly conducted by both interviewers and were randomly assigned to the respondents in all four practices.

\section{Statistical analysis}

We used a random effects ordered probit model to analyze the data, with choice (A, no preference, or B) as the ordinal dependent variable (Train, 2003). Explanatory variables were the differences between the levels of the two alternatives.

For the first objective, to examine whether a WTA and WTP format elicit different preferences and monetary values, for each format a regression model was estimated. Before the regression models were estimated we examined whether preferences were influenced by participants' characteristics (age, sex, experience with hearing aid provision, and income). We included each interaction term in the main attributes model separately, and subsequently included all interaction terms with a statistically significant regression coefficient in the model simultaneously. For all analyses a p-value smaller than 0.05 was considered to be statistically significant. A stepwise backward procedure was used and interaction terms with the highest $p$-value $(>0.05)$ were excluded from the model one at a time. In the presence of interaction the coefficients of the attributes will be dependent on patient characteristics. Differences in the distributions of these characteristics between the groups will therefore influence the comparison of WTA and WTP. To avoid this influence we estimated the models for a group with median characteristics of the total group. The monetary valuation of each attribute was calculated by dividing the regression coefficient of each attribute by the regression coefficient of the cost attribute. This resulted in the marginal willingness to pay or to accept compensation for a change in the level of a certain attribute. As the marginal WTA or WTP is the amount of money that would be required after the change to make ones utility level the same as before the change, we used the 
concept of compensating variation. Confidence intervals for WTA and WTP were calculated using the delta method (Hanemann \& Kanninen, 1998).

For the second objective, to explore whether an income or endowment effect influences the disparity between WTA and WTP, we first examined the disparity for a low income group (below median income) versus a high income group (median income or higher). We used the ratio between WTA and WTP as an indicator for the disparity. We hypothesized that, since WTP is constrained by income whereas WTA is not, the disparity between WTA and WTP would be higher in the low income group as opposed to the high income group. Next, we explored the influence of initial endowments of experience. We examined whether the disparity between WTA and WTP was influenced by the fact that persons had previously experienced hearing aid provision. Previous studies have found that once someone has experienced something, it becomes part of their endowment, and they value it more highly than persons who have not experienced it (Porter \& Macintyre, 1984; Ryan et al., 1998; Ryan \& Ubach, 2003). We therefore hypothesized that in respondents who had experienced hearing aid provision the disparity between WTA and WTP would be higher than in respondents without experience.

With respect to the third objective, we examined the effect of using a WTA format, a WTP format, and a WTA format for losers and a WTP format for gainers to obtain monetary valuation on the net benefit of a new intervention. For this objective losers were respondents who potentially lose utility from implementing the intervention, while gainers were respondents who potentially gain utility from implementing the new intervention. The new, and currently recommended, intervention has all attributes equal to the current organization of hearing aid provision, except for omitting the follow-up at the ENTspecialist (AZOS working group, 2006). With individual regression analyses we checked for each respondent whether he or she preferred the follow-up at the ENT-specialist or not. Respondents who preferred the follow-up at the ENT-specialist were defined as losers from the new intervention, while respondents who preferred no follow-up at ENT were defined as gainers. First we calculated the monetary value for the new intervention using the WTA format. Next, we calculated the monetary value for the new intervention using the WTP format. Third, we calculated the weighted average of the monetary values of the losers in the WTA format and the gainers in the WTP format. To examine the impact of the three different approaches on the net benefit, we subtracted the incremental costs of the new intervention from the incremental benefits. We used the costs of one ENT consultation $(€ 104)$ as an estimate of the cost reduction as a result of the implementation of the new intervention (Oostenbrink et al., 2004). Data were managed in SPSS 12.0.1 and analyzed using STATA 9. 


\section{Results}

\section{Respondents}

Between August and December 2006, 402 persons were invited to participate in the survey. Of them, $300(75 \%)$ agreed to be interviewed. Both versions of the questionnaire were completed by 150 participants each. Nine participants $(3 \%)$ did not answer the dominant pair correctly and were therefore excluded from the analysis. Table 5.2 shows the characteristics of the participants. Completers of the WTA questionnaire had a median age of 72 years (range 18-95), while completers of the WTP questionnaire had a median age of 68 years (range 19-92). This difference was statistically significant (Mann-Whitney $U$ test, p-value 0.02). Although slightly different, the out-of-pocket payments for the hearing aid were not statistically significantly different between the groups (Mann-Whitney $U$ test, p-value 0.746). Also for the other characteristics there were no statistically significant differences between the groups.

There were $31 \%$ missing values on family income (Table 5.2). Missing value imputation by using the regression method resulted in a large standard error. As we expected these missing values not to be random, but to occur more often in persons with higher income, we decided not to use income in the analyses. Alternatively, we used the participant's educational level as a proxy for income, as educational level was correlated with income (Spearman's rho 0.55, p-value $<0.0001$ ), and had no missing values.

\section{WTA versus WTP}

The final sample for analysis consisted of 291 participants, resulting in 4656 choice observations, equally divided over the two formats (WTA 2336, WTP 2320). Table 5.3 shows the final regression models for both formats. The signs and weights of the coefficients did not differ between the models, except for the cost attribute ( $p$-value < 0.0001). The coefficients were all statistically significantly different from zero, except for the attribute regarding the initial assessment at the dispenser in the WTP format.

Next, we compared marginal WTA and WTP (Table 5.3). Participants preferred the initial assessment at a private dispenser. In the WTA format the monetary valuation for the initial assessment at a private dispenser was 3.2 times higher than in the WTP format ( $p$-value 0.21 ). In the WTA format a $10 \%$ increase in accuracy was valued 3.0 times higher than in the WTP format ( $p$-value 0.01). Regarding duration, the WTA format elicited a 1.7 times higher monetary value for two months shorter duration than the WTP format ( $p$-value 0.47). The WTA format elicited a monetary value for the follow-up at the ENT-specialist that was 3.0 times higher than in the WTP format (p-value 0.005). 
Table 5.2 Characteristics of participants for each format and for the total group of participants. Values are numbers (percentages) unless stated otherwise

\begin{tabular}{|c|c|c|c|c|c|c|}
\hline \multirow{2}{*}{$\begin{array}{l}\text { Characteristic } \\
\text { Age (years) }\end{array}$} & \multicolumn{2}{|c|}{$\begin{array}{r}\text { WTA format } \\
(n=146)\end{array}$} & \multicolumn{2}{|c|}{$\begin{array}{r}\text { WTP format } \\
(n=145) \\
\end{array}$} & \multicolumn{2}{|c|}{ Total $(n=291)$} \\
\hline & & & & & & \\
\hline Mean (standard deviation) & 71.0 & (10.7) & 67.6 & $(13.1)$ & 69.3 & $(12.0)$ \\
\hline Median (range) & $72.4^{*}$ & $(18-95)$ & $69.4^{*}$ & $(19-92)$ & 71.3 & $(18-95)$ \\
\hline Male & 85 & $(58 \%)$ & 83 & $(57 \%)$ & 168 & $(58 \%)$ \\
\hline \multicolumn{7}{|l|}{ Education } \\
\hline No education & 2 & $(1 \%)$ & 2 & $(1 \%)$ & 4 & $(1 \%)$ \\
\hline Primary school & 35 & $(24 \%)$ & 26 & $(18 \%)$ & 61 & $(21 \%)$ \\
\hline Lower education & 32 & $(22 \%)$ & 36 & $(25 \%)$ & 68 & $(23 \%)$ \\
\hline Junior general secondary education & 29 & $(20 \%)$ & 23 & $(16 \%)$ & 52 & $(18 \%)$ \\
\hline Senior secondary vocational education & 15 & $(10 \%)$ & 18 & $(12 \%)$ & 33 & $(11 \%)$ \\
\hline Senior general secondary education & 13 & $(9 \%)$ & 10 & $(7 \%)$ & 23 & $(8 \%)$ \\
\hline Higher professional education & 14 & $(10 \%)$ & 25 & $(17 \%)$ & 39 & $(13 \%)$ \\
\hline University & 6 & $(4 \%)$ & 5 & $(3 \%)$ & 11 & $(4 \%)$ \\
\hline \multicolumn{7}{|l|}{ Family income } \\
\hline$€ 450-1100$ & 18 & $(12 \%)$ & 13 & $(9 \%)$ & 31 & $(11 \%)$ \\
\hline$€ 1100-1600$ & 28 & $(19 \%)$ & 30 & $(21 \%)$ & 58 & $(20 \%)$ \\
\hline$€ 1600-2000$ & 21 & $(14 \%)$ & 12 & $(8 \%)$ & 33 & $(11 \%)$ \\
\hline$€ 2000-3000$ & 23 & $(16 \%)$ & 24 & $(17 \%)$ & 47 & $(16 \%)$ \\
\hline$>€ 3000$ & 16 & $(11 \%)$ & 16 & $(11 \%)$ & 32 & $(11 \%)$ \\
\hline Don't know & 14 & $(10 \%)$ & 11 & $(8 \%)$ & 25 & $(9 \%)$ \\
\hline Not willing to answer & 26 & $(18 \%)$ & 39 & $(27 \%)$ & 65 & $(22 \%)$ \\
\hline Previously experienced hearing aid provision & 96 & $(66 \%)$ & 88 & $(61 \%)$ & 184 & $(63 \%)$ \\
\hline \multicolumn{7}{|l|}{ Out-of-pocket payment for the hearing aid $(€)$} \\
\hline Mean (standard deviation) & 839 & (907) & 911 & (985) & 875 & $(944)$ \\
\hline Median (range) & 605 & $(0-5000)$ & 775 & $(0-6000)$ & 735 & $(0-6000)$ \\
\hline
\end{tabular}

* Statistically significantly different: Mann-Whitney $U$ test; $\mathrm{p}<0.05$ 


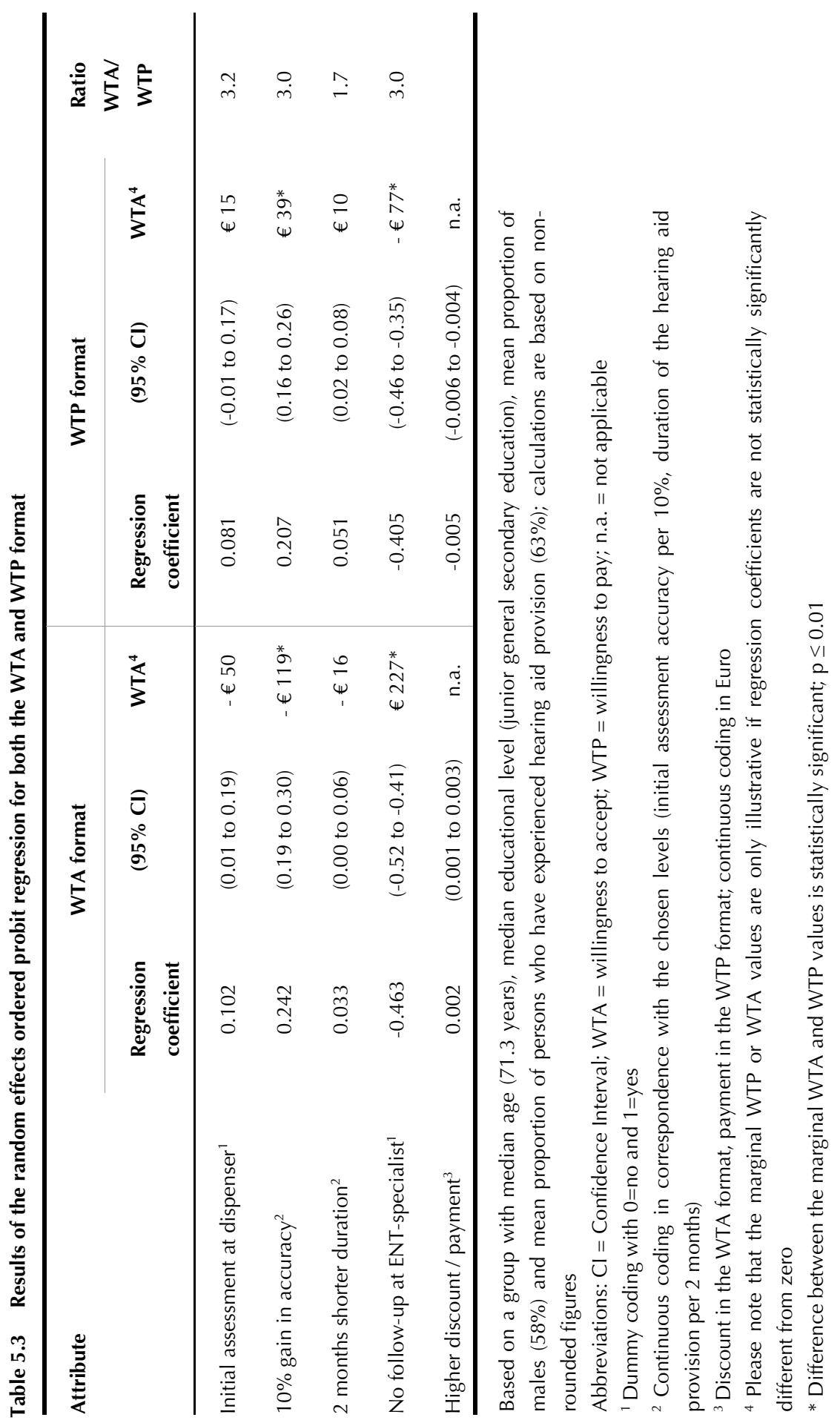




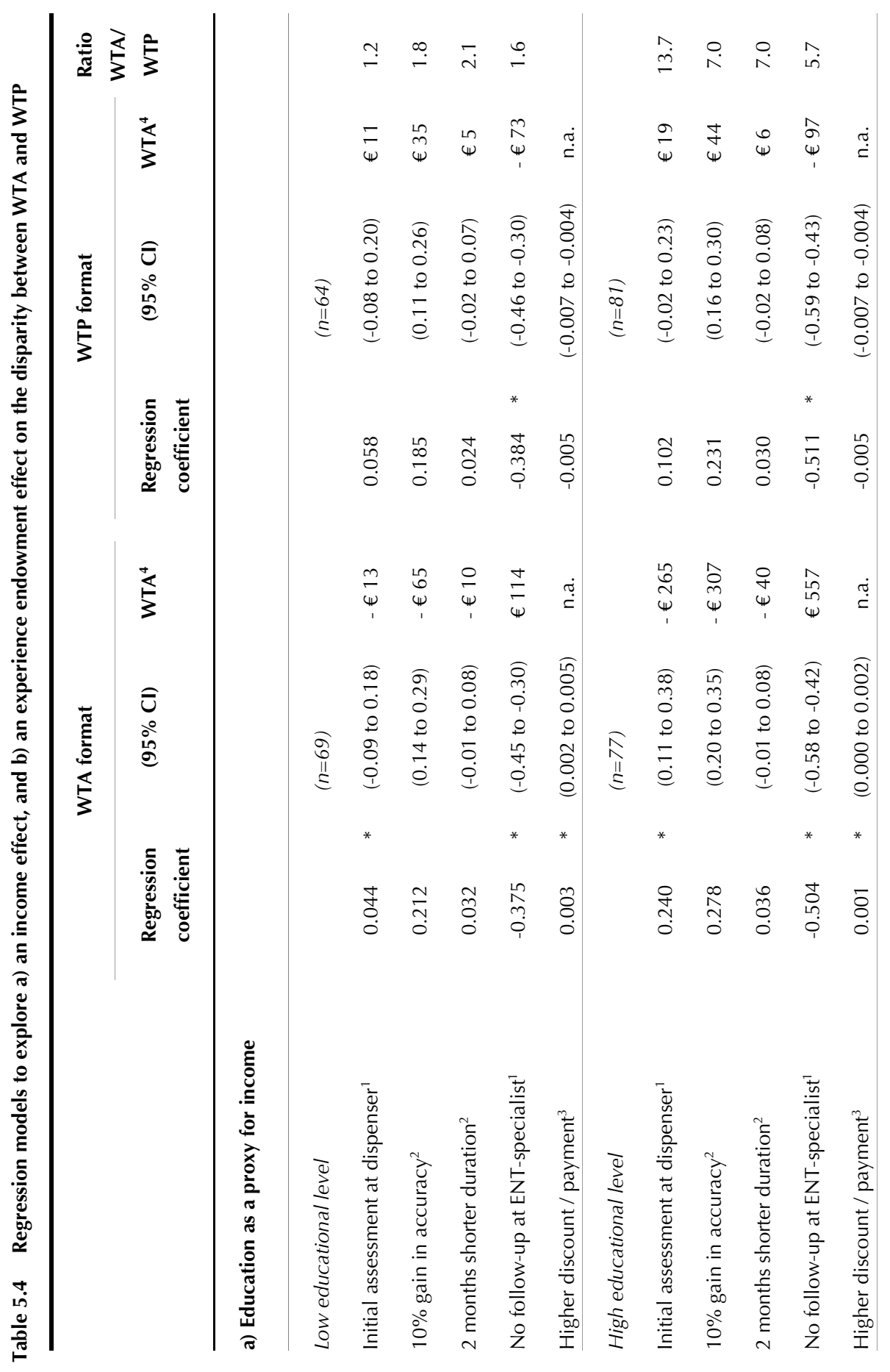




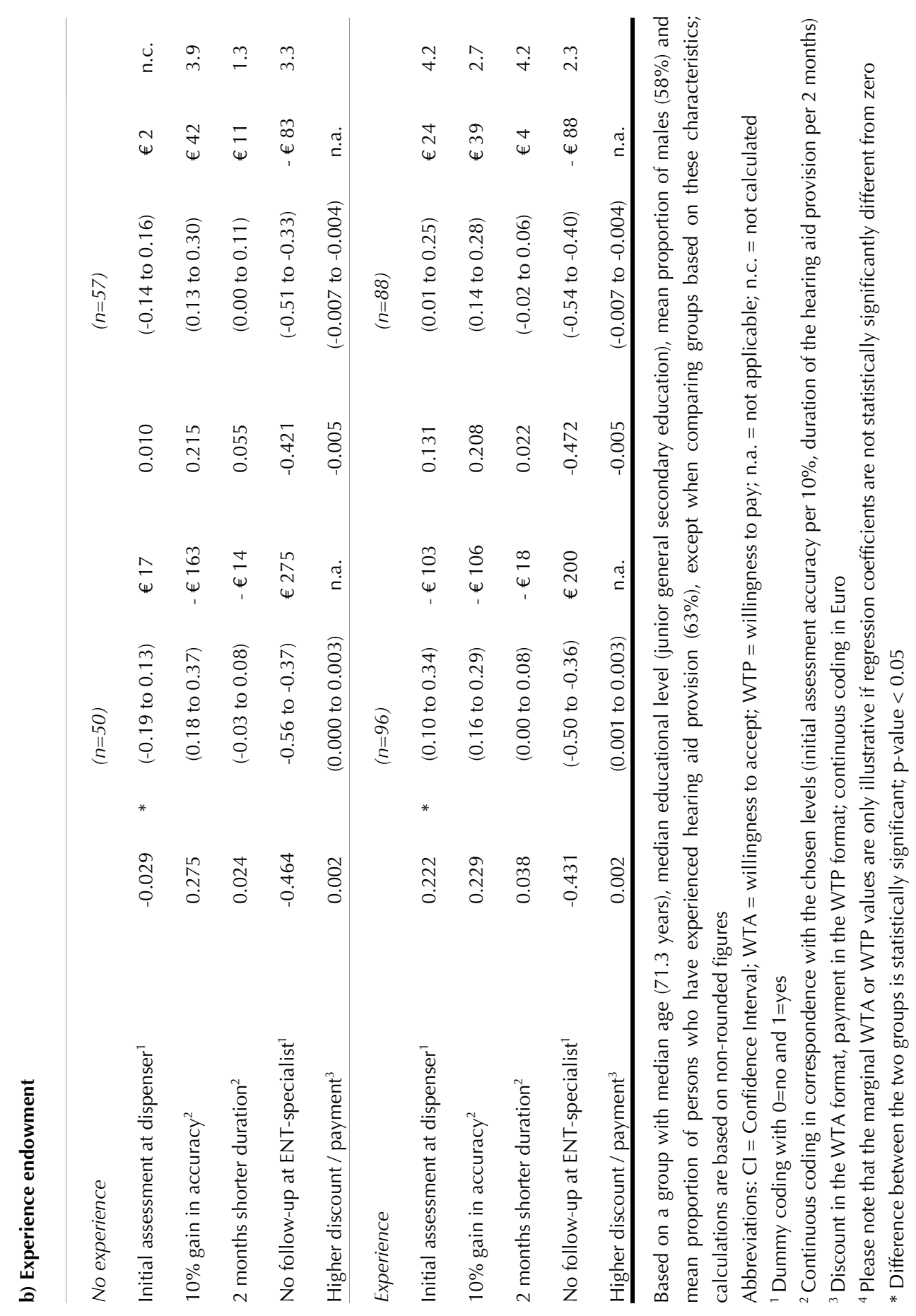




\section{Exploring possible explanations}

We found an education effect, but in the opposite direction of what we hypothesized. The disparity between WTA and WTP was higher in the high educational group (Table 5.4a). This was due to the fact that an education effect only occurred in the WTA format, where participants with a higher educational level found the discount considerably, and statistically significantly, less important than participants with a lower educational level.

We did not find that the disparity between WTA and WTP was higher in persons with experience than in those without experience with hearing aid provision (Table 5.4b). It was inconclusive which group overall showed a higher disparity, as the disparity varied across the attributes. We did find that experienced respondents preferred the initial assessment at the dispenser, while respondents who were inexperienced had no statistically significant preference.

\section{Effect of different formats on the net benefit}

When using the WTA format, the monetary valuation of the new intervention amounts to $€ 227$ (Table 5.5). Use of the WTP format resulted in a monetary valuation of - $€ 77$ for the new intervention. Third, when the WTA format is used for potential losers $(n=118)$ and the WTP format for potential gainers $(n=24)$, monetary values were $€ 76$ for the gainers versus $€ 414$ for the losers. This resulted in a weighted average of - $€ 331$ for the new intervention. All three approaches result in a negative benefit for the new intervention. Given the cost reduction of $€ 104$, this would result in a monetary net benefit of $€ 27$ when using only the WTP format. As this is a positive net benefit, based on the WTP results one would recommend implementation of the new intervention. The other two approaches would result in a negative net benefit (- $€ 123$ and - $€ 227$ respectively), based on which one would recommend not to implement the new intervention.

Table 5.5 Monetary valuation of the new intervention, elicited with a WTA format, WTP format, and a WTA format for losers and WTP format for gainers, and net benefit

\begin{tabular}{lrrrrr}
\hline Format & $\mathbf{N}$ & $\begin{array}{r}\text { Monetary } \\
\text { valuation }\end{array}$ & $(\mathbf{9 5} \% \mathbf{C l})$ & $\begin{array}{r}\text { Weighted } \\
\text { average }\end{array}$ & Net benefit* \\
\hline 1) WTA & 146 & $-€ 227$ & $(-€ 332$ to $-€ 123)$ & & $-€ 123$ \\
2) WTP & 145 & $-€ 77$ & $(-€ 93$ to $-€ 61)$ & & $€ 27$ \\
$3)$ WTA for losers & 118 & $-€ 414$ & $(-€ 686$ to $-€ 143)$ & $-€ 331$ & $-€ 227$ \\
WTP for gainers & 24 & $€ 76$ & $(€ 32$ to $€ 119)$ & & \\
\hline
\end{tabular}

Based on a group with median age (71.3 years), median educational level (junior general secondary education), mean proportion of males (58\%) and mean proportion of persons who have experienced hearing aid provision $(63 \%)$

Abbreviations: $\mathrm{Cl}=$ Confidence Interval; WTA = willingness to accept; WTP = willingness to pay

* Net benefit is based on a cost reduction of $€ 104$ 


\section{Discussion}

This study compares WTA and WTP in a discrete choice experiment. We found the cost attribute to have a statistically significantly higher regression coefficient when it was defined as extra payment compared to a discount. Marginal willingness to accept was statistically significantly higher than willingness to pay for both accuracy and the follow-up at the ENT-specialist. In contrast to our expectations, the disparity between WTA and WTP was higher in a high educational group compared to a low educational group. We found that persons with a higher educational level found a discount less important than those with a lower educational level. Respondents who had experienced hearing aid provision did not have a higher disparity between WTA and WTP than those without experience. Implementing the new intervention would only be recommended when using a WTP format to elicit monetary valuations, and would not be recommended when using a WTA format for losers and a WTP format for gainers or only a WTA format.

\section{Interpretation of results}

The fact that we found a disparity between WTA and WTP is not surprising, as it is indisputable that a disparity between WTA and WTP exists (Brown \& Gregory, 1999; Horowitz \& McConnell, 2002). In their review, Horowitz and McConnell (Horowitz \& McConnell, 2002) found WTA/WTP ratios ranging from 1 to 113, with a mean ratio of 7 . In health care these ratios range from 1 to 6.4 (Borisova \& Goodman, 2003; Carthy et al., 1999; O'Brien et al., 1998; Van den Berg et al., 2005b). The disparity found in this discrete choice experiment is within this range of disparities found in health care contingent valuation studies.

Income is thought to influence the disparity between WTA and WTP, as WTP is constrained by income, whereas WTA is not (Brown \& Gregory, 1999). In our study we did not find a higher disparity in a low educational group as opposed to a high educational group. Extra payment was found to be equally important in both groups. Possibly the $€ 100$ range of extra payment we used was not large enough to reach the budget constraint in the low educational group. However, an education effect was found only in the WTA format, where the group with higher education clearly found the discount less important than the group with lower education. The fact that persons with higher education (and hence higher income) value a certain amount of money lower than persons with lower income is not surprising. We however expected to observe this in the WTP format as well, resulting in no influence of educational level on the disparity between WTA and WTP.

Most of the explanations for the disparity between WTA and WTP relate to the difference between buyers who gain a good (in the WTP format) and sellers who lose a good (in the WTA format). These explanations may however only apply to discrete choice experimentation when each choice set consists of the status quo and an alternative scenario. In the present discrete choice experiment we did not use a status quo design. Also, we did not label the scenarios, and scenarios were equal in both formats except for the cost attribute. By doing this we minimized status quo bias and loss aversion (Salkeld et al., 2000). We found no evidence of an experience endowment effect. For one attribute, 
the initial assessment, we found that respondents without experience showed a stronger preference for the real world status quo (initial assessment at ENT-specialist) compared to those with experience. Probably, persons who have already experienced a medical examination for their hearing problems attached less value to consulting a medical specialist for the initial assessment again. Although it was not the focus of our analysis, when calculating the WTA for potential losers of the new intervention, we found that the WTA for losers was higher than the WTA for the total group (Table 5.5). This result may suggest the presence of loss aversion. The loss aversion may not be directly related to what persons had or had experienced, and thus to endowment or property rights, but may be more related to their attitude towards the new intervention. Possibly loss aversion was based more on an intrinsic feeling of loss than on actual losses.

\section{Limitations}

There are some limitations of the present study. First, although our study population was sufficiently large for the main analysis on the disparity between WTA and WTP, the study population was relatively small for exploring income and endowment effect as explanations of the disparity. Because we had to split both samples in two to compare different groups, we found regression coefficients with large standard errors that were not significantly different from zero. Nevertheless, as our second objective was exploratory we decided to show the results and focus on the ratio between WTA and WTP rather than to draw conclusions only on statistically significantly different results. Another limitation of the present study is that we had no information beforehand on whether the participants would gain or lose utility when implementing the new organization of hearing aid provision. It would be interesting to assign the WTA or WTP questionnaire depending on whether participants are gainers or losers. However, since our main objective was to examine whether monetary valuations differ when using a WTA compared to a WTP format, we feared that asking preferences beforehand would influence the results of the discrete choice experiment, as respondents would possibly be more inclined to choose the status quo. Finally, a potential limitation is that we were forced to use educational level as a proxy for income, because of the large number of missing values on income. We additionally performed the analyses of income effect with income to check whether educational level was a valid proxy, and found a similar but stronger effect (results not presented).

\section{Implications}

The present study has some important implications. First, defining the cost attribute in a discrete choice experiment as a discount or payment changes monetary valuations. When using monetary valuation from a discrete choice experiment in a cost-benefit analysis, as shown in the present study, this may even alter policy recommendations. Whether differences between preferences should be incorporated in decision-making has also been discussed by O'Brien et al. (2002), Severens et al. (2005) and Dowie (2004; 2005). As 
patient preferences gain increasing attention in decision making (Ryan, 2004), and patients have preferences over aspects of health care beyond health-related quality of life, discrete choice experiments will probably become increasingly important in health services research. Most discrete choice experiments in health care use the concept of WTP, but WTA has also been used (Telser \& Zweifel, 2006; Ubach et al., 2003; Van der Pol \& Cairns, 1998). Although in other papers it was stated that the inclusion of a cost attribute to elicit monetary valuations with discrete choice experiments should be done with caution (Hanley et al., 2003; McIntosh, 2006; Ratcliffe, 2000; Slothuus Skjoldborg \& Gyrd-Hansen, 2003), to our knowledge no study has paid explicit attention to when the cost attribute should be defined as a payment or a discount. The lack of clarity on how to address the disparity between WTA and WTP in discrete choice experiments probably results from the fact that before the present study the disparity had not yet been examined and, as a result, had not yet been found in discrete choice experiments.

One can address the disparity between WTA and WTP by asking participants beforehand whether they prefer the program that is under evaluation, and assign the WTA or WTP version of the discrete choice experiment accordingly. This would however require the use of a status quo scenario in each choice set (Mclntosh, 2006), which would possibly introduce status quo bias (Salkeld et al., 2000). More importantly, a great advantage of a discrete choice experiment is its flexibility that monetary values can be calculated for any configuration of attributes and levels (Mclntosh, 2006). As gainers for one configuration can be losers for another, when eliciting WTP from gainers and WTA from losers, one can only calculate the monetary valuation of the interventions where this distinction between gainers and losers holds, which decreases this flexibility.

It has been stated that WTP is preferred over WTA in contingent valuation studies (Arrow et al., 1993; Bayoumi, 2004), since WTP is said to be more valid and more conservative than WTA (Arrow et al., 1993). In the present study however, the WTP for the new intervention resulted in a negative WTP. This indicates that for the new intervention, the use of a WTA format may have been more appropriate. The net benefit analyses showed that if the new intervention would lead to a cost reduction of $€ 104$, implementation of the intervention would only be recommended when the monetary valuation was elicited in the WTP format. This clearly illustrates that using a WTA format is more conservative when the majority of respondents are losers.

\section{Conclusions}

The results of this discrete choice experiment confirm that WTA exceeds WTP. This has consequences when using the discrete choice results in a cost-benefit analysis. It is therefore important that there is consensus on when to define the cost attribute in a discrete choice experiment as a payment or a discount. For now, the best option seems to be to choose the most conservative format, which is WTP if (the majority of) respondents are potential gainers, and WTA if (the majority of) respondents are potential losers. However, 
as stated, this may affect the flexibility of discrete choice experiments to calculate monetary values for different configurations of interest within a cost-benefit analysis.

Now that we have demonstrated that the disparity between WTA and WTP also occurs in discrete choice experiments, more research on when to use a payment or a discount is needed before monetary values based on discrete choice experiments can be used in costbenefit analyses. 


\section{Appendix 5.1 Example of a discrete choice question}

\begin{tabular}{|c|c|c|}
\hline Attribute & Alternative A & Alternative B \\
\hline $\begin{array}{l}\text { Performer and accuracy of the initial } \\
\text { assessment }\end{array}$ & ENT-specialist at hospital & $\begin{array}{l}\text { Dispenser at practice, } \\
\text { equally accurate }\end{array}$ \\
\hline Duration of hearing aid provision & 2 months & 4 months \\
\hline Follow-up at ENT-specialist & $\begin{array}{l}\text { Follow-up at ENT- } \\
\text { specialist }\end{array}$ & $\begin{array}{l}\text { No follow-up at ENT- } \\
\text { specialist }\end{array}$ \\
\hline Extra payment / discount ${ }^{1}$ & $€ 20 / € 50$ & $€ 50 / € 20$ \\
\hline Which alternative would you choose? & $\square \mathrm{A}$ & $\square B$ \\
\hline & \multicolumn{2}{|c|}{ I have no preference } \\
\hline
\end{tabular}

${ }^{1}$ Dependent on format: extra payment was used in the WTP format, a discount was used in the WTA format. 



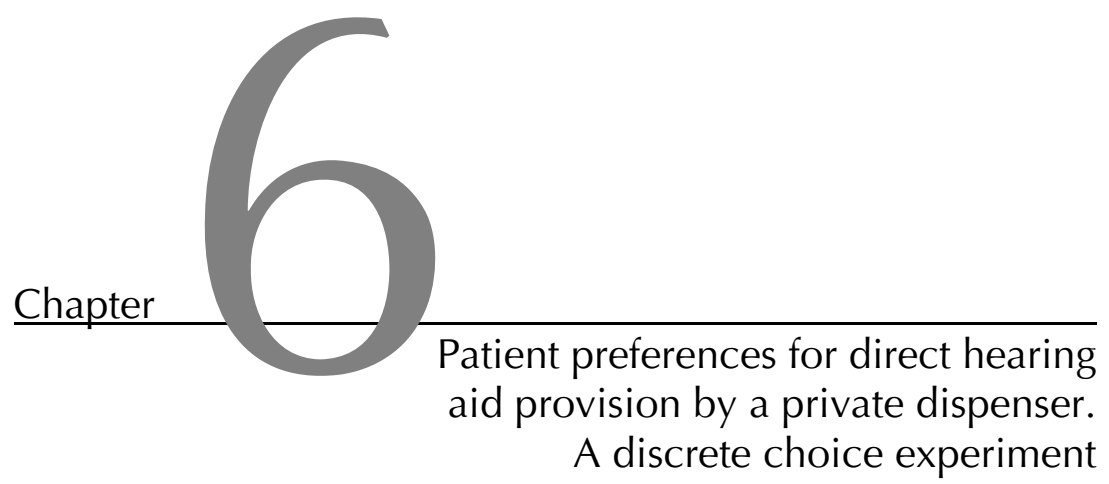

Janneke PC Grutters

Manuela A Joore Alfons GH Kessels

Adrian C Davis

Lucien JC Anteunis

Ear and Hearing, accepted for publication 


\section{Abstract}

\section{Objectives}

Our objective was to elicit patient preferences for transferring elements of hearing aid provision from the medical sector (Ear Nose and Throat specialists and audiological centres) to private hearing aid dispensers, and to understand the trade-offs between different elements of hearing aid provision.

\section{Design}

A discrete choice experiment was administered from 150 hearing-impaired persons in the Netherlands. Mean age was 71 (range 18-95) and 57\% were male.

\section{Results}

Participants preferred the initial assessment at the dispenser, higher accuracy in identifying persons in need of medical care, shorter duration of the total hearing aid provision, and a follow-up at the Ear Nose and Throat (ENT) specialist. They required compensation of at least $€ 17$ per two months extra duration, $€ 54$ for an initial assessment at the ENTspecialist, $€ 119$ per $10 \%$ decrease in accuracy, and $€ 227$ to forgo the follow-up at the ENT-specialist. Preferences were influenced by sex, age, educational level, and experience with hearing aid provision.

\section{Conclusions}

Hearing-impaired persons are receptive to transferring elements of hearing aid provision from the medical sector to private dispensers. Although safety and efficiency issues should also be considered, from the present study we can conclude that in the organization of hearing aid provision hearing-impaired persons prefer an initial assessment at a private dispenser when the dispenser is at least $95 \%$ as accurate as the ENT-specialist, and prefer a follow-up visit at the ENT-specialist. 


\section{Introduction}

Hearing impairment is a common health problem, of which the prevalence heavily increases with age (Davis, 1995). The ageing of the population predicts that the prevalence and, as a result, the health care costs associated with hearing rehabilitation, will continue to increase.

Direct hearing aid provision without medical care has proven to be safe (Abdelkader et al., 2004; Koay \& Sutton, 1996; Swan \& Browning, 1994). In the United Kingdom (UK) general practitioners can refer patients to an ENT-specialist or directly to an audiology department. Some areas have previously encouraged patients to directly visit an audiology department (The British Society of Hearing Aid Audiologists, 2005). Unfortunately this has led to a significant range in patient waits from zero to up to three years (British Academy of Audiology, 2006). Hence, the Health Committee recently carried out an inquiry into audiology services in England, with particular interest in the role of the private sector (Health Committee, 2007a, 2007b). The UK had previously offered as part of its Modernisation of Hearing Aid Services an option for districts to offer an Independent Sector, Hearing Aid Dispenser (a regulated profession), service. This was initially greeted with some skepticism by professionals and hearing aid patients. However the evaluated outcomes showed an equivalence in pilot studies, with considerable patient support in qualitative focus group follow-up. For instance a patient said 'I thought the whole process was very efficient and lean. I felt that the process was very thorough and reassuring'. Those interviewed at one pilot site had nothing but praise for the interpersonal skills of the dispenser. However the experience was not uniform across areas. When asked about standards and ensuring quality, the consensus was that they would always trust the clinician if they looked and acted competently but they agreed that if initiatives like this were more commonplace then the public would need to know that standards were being maintained. Subsequently as noted in the Health Committee there was a very mixed review and experience when the services were rolled out to those who felt that they could help reduce the waiting time for those people who were referred.

In the United States the Food and Drug Administration requires that consumers see a licensed health professional (mostly a doctor or audiologist) before getting a hearing aid (Valente, 2006). Possibly such a system, with private hearing aid dispensers becoming licensed professionals, would be an adequate system in Europe.

In the Netherlands, hearing-impaired persons who seek help first need to visit a general practitioner for referral to an Ear Nose and Throat (ENT) specialist or an Audiological Centre (AC). In an AC a multidisciplinary team offers specialized care to the more seriously impaired with additional complications. Only ENT-specialists and ACs are entitled to prescribe hearing aids. In general the hearing aid is fitted by a private hearing aid dispenser. The patient is entitled to (partial) reimbursement of the hearing aid by their medical insurance only when the hearing aid fitting is approved by the prescriber and the hearing loss exceeds $35 \mathrm{~dB}$, averaged over 1, 2 and $4 \mathrm{kHz}$ in the better ear. Generally, the hearing aid dispenser collects the reimbursement and the patient is billed only for that part of the hearing aid price that exceeds the reimbursement, which ranges from no extra 
payment to over $€ 1000$ per hearing aid. The current organization of hearing aid provision in the Netherlands is costly and may lead to a future shortage of ENT-specialists (Van der Velden \& Hingstman, 2000). Therefore the safety and efficiency of transferring elements of hearing aid provision from the medical sector to private hearing aid dispensers are currently under investigation (AZOS working group, 2006). In order to distinguish 'clients' who are not in need of medical care from 'patients' who require medical attention or treatment of an AC, a set of criteria was developed by a national platform of professionals involved in the care for hearing-impaired persons (Veldhuizen et al., 2002). The criteria for referral relate to otoscopy (i.e. skin aberration, fluid in the auditory canal), audiometry (i.e. asymmetrical hearing loss or sudden deafness) and psychosocial aspects (i.e. communication problems at work). Besides issues of safety, quality, and costs, it is important to consider patient preferences regarding the organization of health care (Ryan, 2004; Ryan et al., 2005). This would increase the chances of a successful implementation of changes in the organization of care. Discrete choice experimentation, an approach for the elicitation of preferences, is widely used in health care and is a useful tool in decisionmaking (Ryan, 2004; Ryan \& Farrar, 2000; Ryan et al., 2001).

Our aim was to elicit patient preferences for transferring elements of hearing aid provision from the medical sector to private hearing aid dispensers, and to understand the trade-offs between these elements of hearing aid provision, using a discrete choice experiment.

\section{Methods}

\section{Discrete choice experiments}

Discrete choice experimentation is a form of conjoint analysis. Conjoint analysis is developed in mathematical psychology and marketing, and is increasingly used in health services research (Ryan \& Farrar, 2000; Ryan \& Gerard, 2003), including hearing research (Meister et al., 2001). Conjoint analysis is grounded in random utility theory and based on the principle that complex decisions are not based on a single criterion or factor, but on several factors 'considered jointly'. Any intervention can be described by its attributes, and the extent to which an individual values an intervention depends on the levels of these attributes (Louviere et al., 2000; Ryan \& Farrar, 2000). In conjoint analysis, respondents are presented with various alternative descriptions of an intervention, each of which represents their attributes at different levels. Respondents are asked to rank the various alternatives (ranking), to rate them (rating) or to choose their most preferred (Louviere et al., 2000). The latter is referred to as discrete choice experimentation (DCE), and is widely used in health care (Ryan, 2004; Ryan \& Farrar, 2000; Ryan \& Gerard, 2003). Conjoint analysis in terms of a ranking experiment has been applied in auditory research to examine the importance of hearing aid attributes by Meister et al. (2001). Much of the appeal of conjoint analysis lays in that it enables us to understand the trade-offs between attributes. When a cost attribute is included, the willingness to pay for a unit change in an attribute can be calculated. Each alternative can then be given a monetary value, which allows for use in a 
cost-benefit analysis, where the costs of a program are compared to its monetary benefits (Drummond et al., 2005). Extensive information on discrete choice experiments can be found elsewhere (Louviere et al., 2000; Ryan \& Farrar, 2000; Ryan \& Gerard, 2003).

\section{Questionnaire design}

Twelve possible attributes of interest with regard to hearing aid provision were identified by experts. In a preliminary survey using five point Likert scales the following six of these attributes were identified by hearing-impaired persons $(n=21)$ as the most important: 1) quality of the hearing aid, 2) the performer of the initial assessment to distinguish between persons in need of medical care (referred to as patients) and persons not in need of medical care (clients), 3) the accuracy of the initial assessment, defined as the probability that patients are referred to an ENT-specialist (sensitivity), 4) the duration of the total hearing aid provision from the initial assessment until the approval of the hearing aid fitting, 5) the follow-up at the ENT-specialist to evaluate the hearing aid fitting, and 6) costs. As the large majority of hearing aids in the Netherlands are prescribed and evaluated by an ENTspecialist (> 85\%), we did not mention the AC in the experiment.

Realistic levels, based on the results of a previous study (AZOS working group, 2006), were assigned to each attribute (Table 6.1).

Table 6.1 Attributes and levels used for discrete choice experiment

\begin{tabular}{ll}
\hline Attributes & Levels \\
\hline Hearing aid quality: & $*$ Good \\
\hline Performer \& accuracy of initial assessment: & $*$ ENT-specialist at hospital \\
& $*$ Dispenser at practice, equally accurate \\
& $*$ Dispenser at practice, 10\% less accurate \\
& $*$ Dispenser at practice, 20\% less accurate \\
\hline Duration: & $* 2$ months \\
& $* 4$ months \\
& $* 6$ months \\
& $* 8$ months \\
\hline Follow-up at ENT: & $*$ Yes \\
& $*$ No \\
\hline Savings: & $*$ No savings \\
& $* € 20$ \\
& $* € 50$ \\
& $* € 100$ \\
\hline
\end{tabular}


Irrespective of how hearing aid provision is organized, the hearing aid should be of good quality. As we expected quality to be a dominant attribute and the study focused on organizational aspects, we only assigned the level 'good' to this attribute. That is, we made sure that for each alternative respondents expected the hearing aid to be of equal quality. As we conservatively assumed the private dispenser to be equally or less accurate than the ENT-specialist, the attributes performer and accuracy of the initial assessment had levels that could not logically be varied independently from each other. Following Louviere et al. (2000), these attributes were combined. Duration of the hearing aid provision was assigned four levels, ranging from 1 month to 7 months. Two levels (yes and no) were assigned to the follow-up at the ENT-specialist.

We expected most participants to attach less value to direct hearing aid provision by the private dispenser, since it restricts access to medical care, and persons often prefer the status quo (Salkeld et al., 2000; Walton et al., 2002). Hence, we defined the cost attribute as willingness to accept, as savings off of the hearing aid price (Drummond et al., 2005). The cost attribute was assigned four levels, equal to two other attributes, to minimize the attribute effect (Ratcliffe \& Longworth, 2002). The range was based on the answers on an open-ended question in the preliminary survey and the levels were based on existing Euro bills.A fractional factorial design was used to reduce the number of choices to the smallest number necessary for an efficient design (Huber \& Zwerina, 1996). We created an orthogonal main effects plan, and subsequently determined 16 choice pairs using shifting (Street et al., 2005). We ordered the attributes in a chronological order, from initial assessment to payment of the hearing aid, to limit the cognitive complexity of the choice tasks, and included the option that respondents were indifferent between the alternatives (no preference). Appendix 6.1 presents an example of a choice set.

Additionally we defined a choice set in which one alternative with a higher discount, higher accuracy, and all other attribute levels equal unquestionably dominated the other, to check whether respondents understood the experiment. The total questionnaire therefore consisted of 17 discrete choice questions.

The questionnaire started with a comprehensive detailed explanation of the attributes and levels to ensure that all participants understood the trade-off features as uniformly as possible. An example preceded the choice sets, to present the technique to the respondents before the beginning of the actual experiment. Three five point Likert scales elicited whether respondents found the questionnaire unclear, difficult or long. Additionally, demographic variables were collected, as well as whether participants had experienced hearing aid provision before.

\section{Survey procedure}

In a pilot study, it was examined whether the participants found all attributes important and whether they were willing to trade between the attributes, to check whether the chosen attributes and levels were appropriate. It was also examined whether the formulation was correct, and whether the participants understood the experiment. The pilot questionnaire, completed by 35 participants, was administered face-to-face by a trained interviewer at a 
private dispenser practice. Based on the results of the pilot study, we slightly adjusted the range of the duration attribute. Respondents found one month to be no realistic level for the total hearing aid provision, as they assumed a month to be too short to adequately fit a hearing aid. Hence, we changed the range of levels for duration to 2 to 8 months. Following the pilot study, two trained interviewers conducted face-to-face interviews at four private hearing aid dispenser practices, chosen to be representative for the region. Participants were persons with hearing complaints visiting the dispenser. As generally all hearing aids in the Netherlands are fitted by private hearing aid dispensers, this affords a study population representative for the Dutch population seeking help for their hearing complaints.

\section{Statistical analysis}

We used a random effects ordered probit model to analyze the data, with choice (A, no preference, B) as the ordinal dependent variable (Train, 2003). Explanatory variables were the differences between the levels of the two alternatives. The attributes 'initial assessment at ENT-specialist' and 'follow-up at ENT-specialist' were analyzed using dummy coding. The levels of the attributes 'initial assessment accuracy' and 'duration' were treated as continuous variables, in correspondence with the chosen levels. Consequently, initial assessment accuracy was analyzed per $10 \%$ and duration of the hearing aid provision was analyzed per 2 months. The levels of the 'savings' attribute were also coded continuously, and the attribute was analyzed per Euro.

Two regression models were estimated. One included the main attributes only, the second model examined how preferences were influenced by participants' characteristics (age, sex, experience with hearing aid provision, and income). For the second model we first included each interaction term in the main attributes model separately. Next, all interaction terms with a statistically significant regression coefficient were included in the model simultaneously. We used a stepwise backward procedure and variables with the highest $\mathrm{p}$ value $(>0.05)$ were excluded from the model one at a time.

The monetary valuation of each attribute was calculated by dividing the regression coefficient of each attribute by the regression coefficient of the cost attribute. This resulted in the marginal willingness to accept compensation for a change in the level of a certain attribute. Confidence intervals for willingness to accept were calculated using the delta method (Hanemann \& Kanninen, 1998).

Additionally we calculated monetary values for three conservative alternative scenarios of hearing aid provision, as opposed to the current situation. In the Netherlands, hearing aid provision currently includes both an initial assessment and a follow-up at the ENTspecialist at a duration of six months. The first scenario, full direct hearing aid provision by the private dispenser, was defined as having both a $10 \%$ less accurate initial assessment and a follow-up at the private dispenser, with a duration of two months. The second scenario had a $10 \%$ less accurate initial assessment at the private dispenser, a duration of four months, and a follow-up at the ENT-specialist. The third scenario had an initial 
assessment at the ENT-specialist, a duration of four months, and a follow-up at the private dispenser.

Data were managed in SPSS 12.0.1 and analyzed using STATA 9.

\section{Results}

\section{Respondents}

Between August and December 2006, 201 persons were invited to participate in the survey. Of them, 150 (75\%) agreed to be interviewed. Table 6.2 shows the characteristics of the participants. Four participants (3\%) did not answer the dominant pair correctly and were therefore excluded from the analysis. Ten participants $(7 \%)$ found the information in the questionnaire unclear. Twenty-four participants (16\%) found the questionnaire difficult, and 67 participants (46\%) found that the questionnaire consisted of (too) many questions.

Table 6.2 Characteristics of participants $(n=150)$. Values are numbers (percentages) unless stated otherwise

\begin{tabular}{|c|c|c|c|}
\hline \multicolumn{2}{|l|}{ Characteristic } & \multicolumn{2}{|c|}{ Descriptive statistic } \\
\hline \multirow[t]{2}{*}{ Age } & Mean (standard deviation) & $71.0(10.7)$ & years \\
\hline & Range & $18-95$ & years \\
\hline Male & & 85 & $(56.7 \%)$ \\
\hline \multirow[t]{4}{*}{ Marital status } & Married & 97 & $(64.7 \%)$ \\
\hline & Widow/ widower & 37 & $(24.7 \%)$ \\
\hline & Not married & 11 & $(7.3 \%)$ \\
\hline & Living together & 5 & $(3.3 \%)$ \\
\hline \multirow[t]{6}{*}{ Education } & No education / primary school & 37 & $(24.7 \%)$ \\
\hline & Lower education & 32 & $(21.3 \%)$ \\
\hline & Junior general secondary education (MAVO) & 33 & $(22.0 \%)$ \\
\hline & Senior secondary vocational education (MBO) & 15 & $(10.0 \%)$ \\
\hline & Senior general secondary education (HAVO / VWO) & 13 & $(8.7 \%)$ \\
\hline & Higher professional education $(\mathrm{HBO})$ / university & 20 & $(13.3 \%)$ \\
\hline \multirow[t]{7}{*}{ Family income } & $€ 450-1100$ & 18 & $(12.0 \%)$ \\
\hline & $€ 1100-1600$ & 28 & $(18.7 \%)$ \\
\hline & $€ 1600-2000$ & 22 & $(14.7 \%)$ \\
\hline & $€ 2000-3000$ & 24 & $(16.0 \%)$ \\
\hline & $>€ 3000$ & 17 & $(11.3 \%)$ \\
\hline & Don't know & 14 & $(9.3 \%)$ \\
\hline & Not willing to answer & 27 & $(18.0 \%)$ \\
\hline \multicolumn{2}{|c|}{ Previously experienced hearing aid provision } & 98 & $(65.3 \%)$ \\
\hline
\end{tabular}




\section{Preferences}

The final sample for analysis consisted of 146 participants, resulting in 2336 choice observations. The coefficients for the attributes were all statistically significantly different from zero (Table 6.3). The signs of the coefficients were positive for all attributes, except for the initial assessment at the ENT-specialist and duration. Duration seemed to be the least important attribute, as a two months longer duration of the hearing aid provision was accepted if the participants would be compensated with at least $€ 17$. Participants preferred the initial assessment at a private dispenser and were willing to accept an initial assessment at the ENT-specialist if they would be compensated with at least $€ 54$. For a $10 \%$ loss in accuracy participants requested compensation of at least $€ 119$. Participants preferred the follow-up at the ENT-specialist and requested compensation of at least $€ 227$ to forgo this follow-up.

Table 6.3 Results of the random effects ordered probit regression* with main attributes only

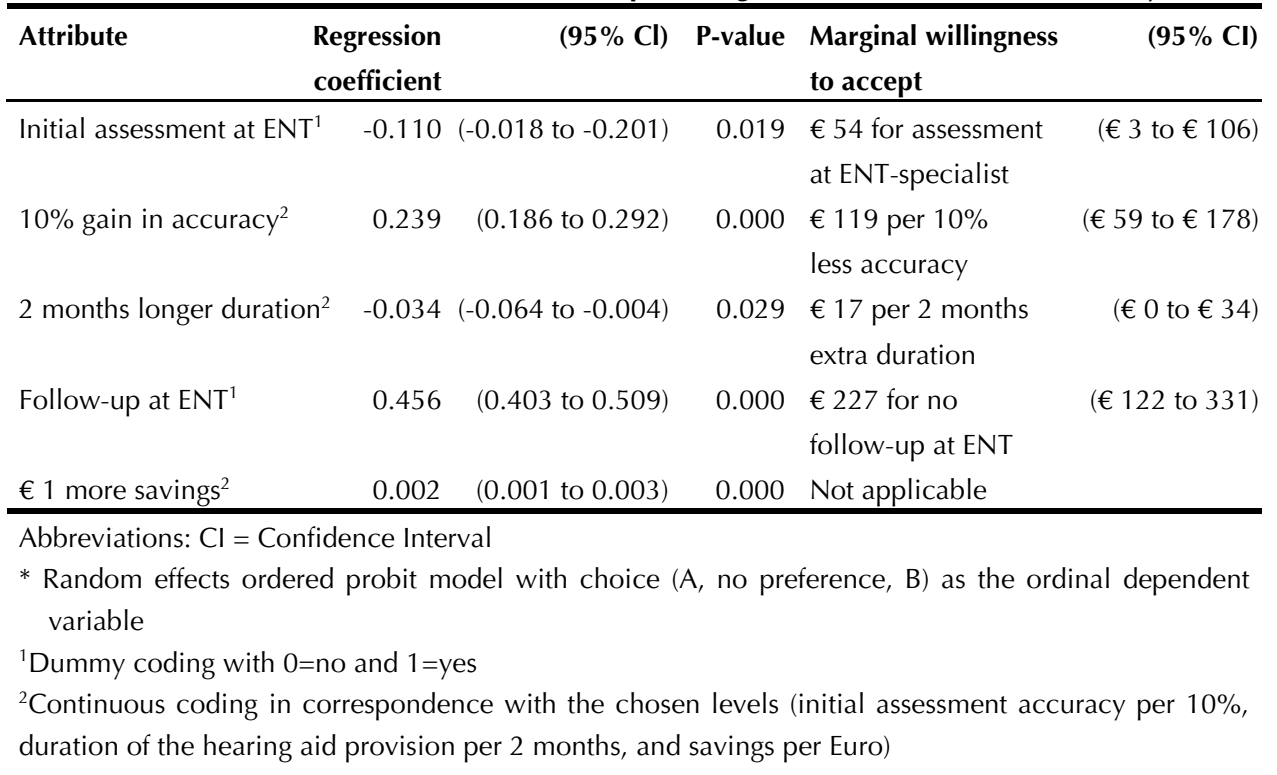

Participants would accept implementation of full direct hearing aid provision by the private dispenser (scenario 1) when they would be compensated with at least $€ 258$ (Table 6.4). For scenario 2 (an initial assessment at the private dispenser, a follow-up at the ENTspecialist and a duration of two months less) they requested compensation of at least $€ 48$. For the final scenario of an initial assessment at the ENT-specialist, a follow-up at the dispenser and two months less duration, they wanted to be compensated with at least $€ 210$. 
Table 6.4 Willingness to accept for three possible scenarios of hearing aid provision

\begin{tabular}{lllll}
\hline Attribute & $\begin{array}{l}\text { Current } \\
\text { situation }\end{array}$ & Scenario 1 & Scenario 2 & Scenario 3 \\
\hline Quality of the hearing aid & good & good & good & good \\
\hline Performer \& & ENT-specialist & private dispenser, & private dispenser, & ENT-specialist \\
accuracy of initial assessment & & $10 \%$ less accurate & $10 \%$ less accurate & \\
\hline Duration & 6 months & 2 months & 4 months & 4 months \\
\hline Follow-up at ENT-specialist & yes & no & yes & no \\
\hline Willingness to accept & - & $€ 258$ & $€ 48$ & $€ 210$ \\
\hline
\end{tabular}

\section{Influence of participant characteristics}

There were $27 \%$ missing values on family income (Table 6.2 ). When analyzing whether preferences were dependent on income, this would result in a strongly reduced number of usable observations. As missing value imputation by using the regression method resulted in a large standard error, we decided not to use income in the interaction model. Alternatively, we examined whether preferences were influenced by a participant's educational level, which was correlated with income (Spearman's Rho 0.62, p-value < 0.001 ), and had no missing values.

Preferences were influenced by age, sex, educational level, and experience with hearing aid provision (Table 6.5). Older participants preferred the initial assessment at the dispenser more strongly and placed less weight on the savings. Women showed a stronger preference for the initial assessment at the dispenser and a weaker preference for the follow-up at the ENT-specialist than men. Participants with a higher educational level placed less weight on the savings, and persons who had previous experience with hearing aid provision preferred the initial assessment at the dispenser more strongly. 
Table 6.5 Results of the random effects ordered probit regression* with main attributes and interactions

\begin{tabular}{|c|c|c|c|}
\hline Attribute & $\begin{array}{l}\text { Regression } \\
\text { coefficient }\end{array}$ & $\begin{array}{r}\text { (95\% Confidence } \\
\text { Interval) } \\
\end{array}$ & $\begin{array}{r}P \text { - } \\
\text { value }\end{array}$ \\
\hline Initial assessment at ENT-specialist ${ }^{1}$ & 0.702 & (0.166 to 1.238$)$ & 0.010 \\
\hline $10 \%$ gain in initial assessment accuracy ${ }^{2}$ & 0.243 & (0.190 to 0.297$)$ & 0.000 \\
\hline 2 months longer duration of hearing aid provision ${ }^{2}$ & -0.034 & $(-0.064$ to -0.003$)$ & 0.031 \\
\hline Follow-up at ENT-specialist ${ }^{1}$ & 0.545 & (0.474 to 0.616$)$ & 0.000 \\
\hline$€ 1$ more savings ${ }^{2}$ & 0.014 & $(0.007$ to 0.021$)$ & 0.000 \\
\hline Age $^{2 *}$ initial assessment at dispenser $^{1}$ & 0.007 & (0.000 to 0.148$)$ & 0.043 \\
\hline Age $^{2 *}$ savings $^{2}$ & -0.0001 & $(-0.0002$ to -0.0000$)$ & 0.006 \\
\hline Sex $^{3} *$ initial assessment at dispenser ${ }^{1}$ & 0.210 & (0.058 to 0.363$)$ & 0.007 \\
\hline Sex ${ }^{3} *$ follow-up at ENT-specialist ${ }^{1}$ & -0.187 & $(-0.294$ to -0.080$)$ & 0.001 \\
\hline Educational level $^{4} *$ savings $^{2}$ & -0.001 & $(-0.002$ to -0.001$)$ & 0.000 \\
\hline Experience $^{1 *}$ initial assessment at dispenser $^{1}$ & 0.281 & (0.121 to 0.440$)$ & 0.001 \\
\hline
\end{tabular}

* Random effects ordered probit model with choice (A, no preference, B) as the ordinal dependent variable

${ }^{1}$ Dummy coding with $0=$ no and $1=y e s$

${ }^{2}$ Continuous coding in correspondence with the chosen levels (initial assessment accuracy per 10\%, duration of the hearing aid provision per 2 months, savings per Euro, and age in years)

${ }^{3}$ Dummy coding with $0=$ male and $1=$ female

${ }^{4}$ Categories with higher number representing higher education 


\section{Discussion}

To our knowledge, this is the first study regarding patients' preferences for the organization of hearing aid provision. In general, participants preferred hearing aid provision with an initial assessment at the hearing aid dispenser with the same accuracy as at the ENTspecialist, a duration of two months, and a follow-up at the ENT-specialist. They requested compensation of at least $€ 17$ for two months extra duration. For an initial assessment at the ENT-specialist participants requested compensation of at least $€ 54$. A $10 \%$ decrease in accuracy was accepted when they would receive compensation of at least $€ 119$. Participants requested compensation of at least $€ 227$ to forgo the follow-up at the ENTspecialist. Preferences were influenced by sex, age, educational level, and experience with hearing aid provision.

\section{Limitations}

Discrete choice experiments are cognitively challenging (Louviere et al., 2000). As our study population was on average 71 years old we were aware of the risk that participants might not understand the questionnaire, causing invalid preferences. The questionnaires were therefore administered in face-to-face interviews, and a validity check was added. Four participants (3\%) answered the dominant pair question incorrectly, indicating invalid preferences, and were therefore excluded from the analysis. We found no evidence that the remaining 146 participants had invalid preferences. To limit the cognitive demand of the experiment, we ordered the attributes chronologically, thus reflecting the normal process of hearing aid provision. Although Kjaer et al. (2006) recently suggested that an ordering effect of the attributes exists, we expected that when ordering the attributes randomly, the increase in cognitive demand would outweigh the advantages of minimizing the ordering effect.

Although there is no consensus on the maximum number of choice sets a respondent can cope with, the 18 sets we defined might be perceived as a lot. This is emphasized by the fact that almost half of the participants (46\%) found that the questionnaire consisted of (too) many questions. There were no missing values however, probably due to the face-to-face interview administration.

Although the questionnaire started with a comprehensive detailed explanation of the attributes, we are aware that accuracy may have been a difficult concept for the respondents to understand. That is, it is difficult to fully understand the consequences of mistakenly identifying a patient as a client, withholding him medical care. The importance of the accuracy attribute might therefore be over- or underestimated. However, as the confidence interval surrounding the regression coefficient of the accuracy attribute is not very large, there is no indication that the concept of accuracy was actually misunderstood. Finally, we included non-traders in the analysis. Respondents were classified as not willing to trade when they consequently chose an alternative in terms of one attribute, irrespective of the levels of the other attribute. In other studies non-traders are often excluded (Ratcliffe \& Buxton, 1999; Ryan \& Farrar, 2000; Ryan \& Hughes, 1997), but Lancsar and Louviere 
(2006) recently advocated not to remove them, as non-traders are of particular interest to decision makers and random utility theory can cope with these preferences. We did estimate an additional regression model without the seventeen non-traders (12\%), and found similar results.

\section{Implications}

Participants preferred hearing aid provision with a shorter duration. Direct hearing aid provision by private dispensers could lead to a decrease in patient waits and a reduction in the number of consultations, resulting in a shorter duration.

The participants also preferred to initially visit a dispenser instead of going to an ENTspecialist in the hospital. However, they value the initial assessment at the dispenser similar to a $5 \%$ gain in accuracy of that initial assessment. This indicates that hearing-impaired persons only prefer to initially visit a private dispenser when he is at least $95 \%$ as accurate as the ENT-specialist. To enhance the accuracy of the private hearing aid dispenser, a set of referral criteria was developed (Veldhuizen et al., 2002). Additionally a training program, corresponding to the referral criteria, was especially designed for the purpose of the initial assessment. The main topics of the training program are audiometry and otoscopy. However, until now this training program has only been applied in an evaluative context. More research is needed to determine whether private dispensers are able to accurately distinguish between patients and clients, and which skills they require for this purpose.

Since private dispenser practices are often more accessible than hospitals, hearingimpaired persons may seek amplification sooner when they do not need to visit a hospital. This is emphasized by the fact that older persons preferred the initial assessment at the dispenser more strongly. Participants who had previously experienced hearing aid provision also had stronger preferences for the initial assessment at the dispenser. Apparently persons attach less value to consulting a medical specialist for the initial assessment once they have had a medical examination for their hearing problems before.

Our results suggest that hearing impaired persons find the follow-up at the ENT-specialist very important, as they request high compensation to forgo this follow-up. They even request a higher compensation ( $€ 227$ ) than the actual cost of a follow-up at the ENTspecialist (at maximum $€ 104$ ) (Oostenbrink et al., 2004). A possible disadvantage of direct hearing aid provision by private dispensers is that the prescriber of the hearing aid financially benefits from selling hearing aids. This may result in over-provision of hearing aids. This disadvantage may possibly be cancelled out when an independent evaluation of the hearing aid remains available. In the UK it was experienced that persons prefer a telephone follow-up rather than a clinical face-to-face follow-up in some categories. More research is needed on whether this is a safe and feasible alternative.

Participants requested the least compensation for a scenario of hearing aid provision with a $10 \%$ less accurate initial assessment at the private dispenser and a follow-up at the ENTspecialist. When the assessment would be only $5 \%$ less accurate than at the ENT-specialist, they would even request no compensation. This indicates that from a patient perspective one should strive for implementation of hearing aid provision with a follow-up at the ENT- 
specialist and an initial assessment at the private dispenser, if possible with accuracy similar to that of the current hearing aid provision.

\section{Conclusion}

The possibility of direct hearing aid provision by private dispensers may save costs, decrease patient waits, and may even break down barriers delaying hearing-impaired persons to seek help. This has certainly been the case in England, but there were concerns about the quality of the hearing aid provision.

Discrete choice experimentation seems to be a feasible instrument to measure preferences in an elderly population. Besides patient preferences, safety and efficiency are important issues to consider when changing the organization of hearing aid provision. From the present study however we conclude that Dutch hearing-impaired persons are receptive to transferring elements of hearing aid provision from the medical sector to private hearing aid dispensers. Whether they prefer the initial assessment at the private dispenser or at the ENT-specialist depends on the accuracy of the dispenser to distinguish between patients and clients. Participants prefer a private dispenser when he is at least $95 \%$ as accurate as the ENT-specialist, prefer a follow-up at the ENT-specialist, and request a higher compensation to forgo this follow-up than its actual costs. 


\section{Appendix 6.1 Example of a discrete choice question}

\begin{tabular}{lll}
\hline Attribute & Alternative A & Alternative B \\
\hline Quality of the hearing aid & Good & Good \\
Performer and accuracy of the initial & ENT-specialist at & Dispenser at practice, equally \\
assessment & hospital & accurate \\
Duration of hearing aid provision & 2 months & 4 months \\
Follow-up at ENT-specialist & Follow-up at ENT- & No follow-up at ENT- \\
& specialist & specialist \\
Savings & $€ 20$ & $€ 50$ \\
& & \\
Which alternative would you choose? & $\square$ A & $\square$ \\
& & $\square$ I have no preference \\
\hline
\end{tabular}



Part III

Integration of evidence 



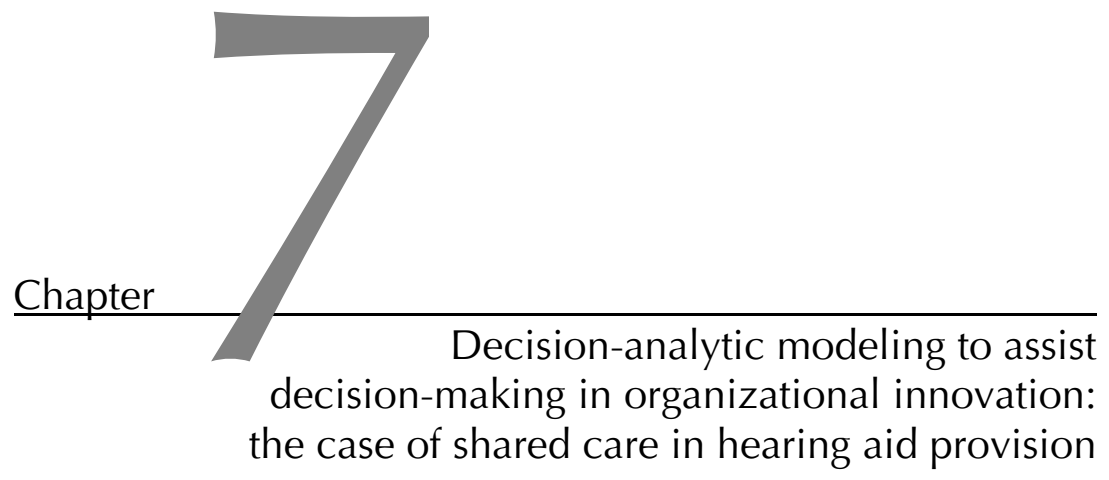

Janneke PC Grutters

Manuela A Joore Frans van der Horst

Robert J Stokroos

Lucien JC Anteunis

Submitted 


\section{Abstract}

\section{Objective}

To examine the long-term cost-effectiveness of, and inform the research agenda on, alternative forms of shared care in hearing aid provision.

\section{Study setting/data sources}

Available evidence from a prospective study, a patient cohort study, published literature and expert opinion was synthesized.

\section{Study design}

A probabilistic decision-analytic Markov model with health states based on hearing aid use and pathology was constructed.

\section{Data collection/extraction methods}

We modeled the long-term cost-effectiveness of (different forms of) shared care as opposed to the current organization of hearing aid provision, for persons aged 50+. Additionally we assessed the value of additional research, and for which topics further research is most valuable.

\section{Principal findings}

The current organization of hearing aid provision as compared to shared care is more effective, and had a higher probability of being cost-effective (54\%). It is however worthwhile to perform additional research, especially on whether persons seek help for their hearing complaints sooner in a shared care pathway and whether the dispenser is capable of detecting pathology.

\section{Conclusions}

Based on the current evidence shared care, in any form, should not be implemented just yet, and it is worthwhile to perform additional research to reduce uncertainty surrounding this decision. 


\section{Introduction}

In several health care sectors tasks of medical specialist are transferred to other professions, sometimes even outside the medical sector (Adams, 2004; Nancarrow \& Borthwick, 2005; Norris, 2001; Stevens et al., 2007). This paper illustrates how decision-analytic modeling can assist decision-making in such organizational innovations. As a case, we examined shared care in hearing aid provision.

Until recently, all hearing-impaired persons in the Netherlands needed a prescription and approval of their hearing aid by an Ear Nose and Throat (ENT) specialist or Audiological Centre (AC). The hearing aid was generally fitted by a private hearing aid dispenser. As only a small proportion of hearing-impaired adults require management other than hearing aids, it is advocated that not all persons with hearing complaints need to be examined by an ENT-specialist or AC (Swan \& Browning, 1994). Also, the ageing of the population increases the costs associated with hearing rehabilitation, and significant patient waits exist in for example the UK (British Academy of Audiology, 2006). Hence, increasing attention is currently paid to shared care initiatives in hearing aid provision. These initiatives include direct hearing aid provision by private dispensers without prescription or approval. However, the problem is that little evidence exists regarding shared care in hearing aid provision. It is unsure whether private dispensers are capable of identifying the subgroup of persons who do require medical care (safety). Also, it is unclear whether the transfer of tasks will reduce costs while maintaining the quality of the hearing aid fitting (efficiency). Especially in an early stage, organizational innovation can be designed in various alternative ways, which makes it difficult to perform trials to assess safety and efficiency. However, the lack of evidence did not mean that decisions were postponed. In fact, some health insurance companies in the Netherlands already allow private dispensers to take over tasks from ENT-specialists and ACs.

It is better to inform a decision with the available evidence than without any evidence at all (Weinstein et al., 2003). Decision-analytic modeling is a useful tool to synthesize available evidence, and is increasingly used to estimate the safety and efficiency of health care interventions (Weinstein et al., 2003). It is not intended to reveal scientific truth (Weinstein, 2006), but to guide decision-making. Data from different sources can be combined to inform decision making under conditions of uncertainty (Briggs et al., 2006; Fenwick et al., 2006). Additionally, one can assess the value of additional research, and specifically what type of additional evidence would be most valuable. It can therefore assist in making a twofold decision: 1) whether or not an intervention should be implemented, given the current evidence, and 2) whether it is worthwhile to perform additional research to reduce the uncertainty surrounding the first decision (Claxton et al., 2002).

The current paper aims to examine the long-term cost-effectiveness of (different forms of) shared care as opposed to the current organization of hearing aid provision, for persons aged 50 years and older. Additionally, we assessed the value of additional research, and for which topics further research is most valuable on this subject. 


\section{Methods}

\section{Model description}

A Markov model was constructed with mutually exclusive health states. The model simulated the course of events in a hypothetical cohort of persons with hearing complaints, aged 50 years or older. In the model, as time progresses, persons move between the different health states according to a set of transition probabilities. The cycle length of the model was set to one year, with a lifetime time horizon.

The model was constructed to compare the expected lifetime costs and effects of shared care alternatives with the lifetime costs and effects of the current organization of hearing aid provision in the Netherlands. The current organization, where persons seek help with the general practitioner (GP) and ENT-specialist or AC, and all persons fitted with a hearing aid have an evaluation of their hearing aid fitting at the ENT-specialist or AC, is referred to as the 'current pathway' (Table 7.1). We defined three shared care pathways: a 'total direct pathway', a 'triage pathway' and a 'follow-up pathway'. In the 'total direct pathway' persons seek help with a private dispenser. The private dispenser uses a checklist with referral criteria to perform a triage to distinguish between patients (suspected of pathology) and clients (no pathology). For clients, as opposed to patients, there is no need to visit the ENT-specialist or AC. In the 'triage pathway' persons seek help with the private dispenser, but all persons fitted with hearing aids have an evaluation at the ENT-specialist or AC. In the follow-up pathway persons always seek help with their GP, but for clients there is no need for an evaluation of the hearing aid fitting by the ENT-specialist or AC.

The analysis was performed from the societal perspective, and all costs were reported in Euros ( $€ 1$ is US \$1.25, average 2006 conversion rate). Future costs and effects were discounted to their present value by a rate of $4 \%$ and $1.5 \%$ respectively, according to Dutch guidelines (Health Care Insurance Board, 2006). Data sources for the parameters in the model were a prospective study (valid $n=269$ ), a patient cohort study $(n=1000)$, published literature and, if no other source was available, expert opinion. Unless stated otherwise, expert opinion was provided by the authors through an informal process.

Table 7.1 Alternative pathways of hearing aid provision

\begin{tabular}{lll}
\hline Alternative & Initial assessment & Follow-up at ENT-specialist \\
\hline Current pathway & GP \& ENT-specialist / AC & Always \\
Total direct pathway & Private dispenser & Only for patients \\
Triage pathway & Private dispenser & Always \\
Follow-up pathway & GP \& ENT-specialist / AC & Only for patients \\
\hline
\end{tabular}

Abbreviations: GP = General Practitioner; ENT $=$ Ear, Nose and Throat; $\mathrm{AC}=$ Audiological Centre 


\section{Model construction}

\section{Health states}

Health states were based on two aspects, being hearing aid use and pathology. For the first aspect health states were 'no hearing aid user', 'hearing aid user' and 'ex hearing aid user'. Hearing aid users were subdivided into two health states: good quality hearing aid fitting and poor quality hearing aid fitting. For the second aspect, we first identified pathology that was associated with hearing loss: acoustic neuroma, chronic otitis media, otosclerosis, otitis externa, Ménière's disease, genetic hearing loss and psychosocial problems. We then excluded pathology with symptoms of such nature that they would not be missed by the dispenser or by the patients themselves (otitis externa, Ménière's disease). Subsequently we excluded pathology where no direct health consequences from missed treatment as a result of a missed diagnosis were expected (genetic hearing loss). Finally, we included the following pathology health states in the model: 'chronic otitis media', 'acoustic neuroma', 'otosclerosis', and 'psychosocial problems' (Figure 7.1). Pathology states were either detected or undetected. For detected acoustic neuroma we included an 'after treatment' (either surgery or radiotherapy) health state. The final absorbing state was 'death'.

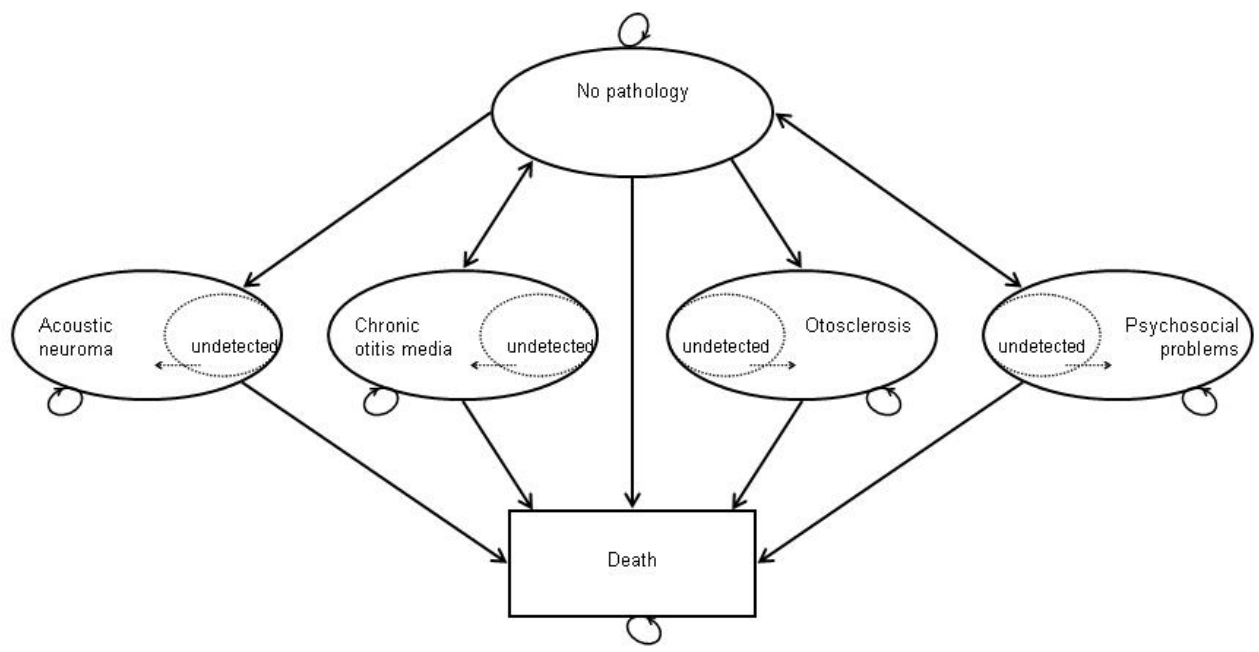

\section{Figure 7.1 Simplified schematic diagram of the Markov model*}

*All health states except detected otitis media are further divided into hearing aid users and non-users (and ex-users in the no pathology state). For detected acoustic neuroma before and after treatment states are distinguished. 


\section{Model assumptions}

We assumed that in the total direct pathway and triage pathway all persons with hearing complaints would seek help with the private dispenser. Also, we assumed that in the total direct pathway and follow-up pathway no dispensers would refer persons without pathology (clients) to an ENT-specialist or AC for evaluation of the hearing aid fitting. This means that we assumed perfect adherence to the shared care pathways, or perfect implementation. Another model assumption was that all persons with pathology (patients) would seek help, and that the GP would refer all patients to an ENT-specialist, so that in the current pathway there would be no undetected pathology. Also, we expected the GP to refer all clients who were potential hearing aid candidates to the ENT-specialist. Finally, we expected hearing aid fittings to be of good quality when they were evaluated by an ENTspecialist or AC.

\section{Probabilities}

All probabilities are listed in Table 7.2. Age-specific incidence figures were found only for acoustic neuroma (Propp et al., 2006). For chronic otitis media and otosclerosis we used incidence figures of the general population (Cummings, 2005). As the typical age of onset of the hearing loss associated with otosclerosis is between 30 and 40 years (Cummings, 2005), we assumed that the incidence after the age of 50 would be at maximum one third of the incidence of the general population (expert opinion). We found no published literature on the incidence of psychosocial problems, and therefore used a database of 1000 randomly selected patients consulting for hearing problems with an ENT-specialist in a community hospital in the Netherlands in 2002 (Duijvestijn et al, unpublished data). Of the 744 persons aged 50 years and older, 13 (1.7\%) were referred to an AC for specialized psychosocial care.

Shared care in hearing aid provision might lower barriers to help-seeking for hearing complaints. In a survey, $24 \%$ of the persons with hearing complaints reported that they would be inclined to obtain a hearing aid sooner when they did not have to visit their GP, an ENT-specialist or AC first (Grutters et al., 2007b). Based on expert opinion, we made a conservative estimation of $10 \%$ extra help-seeking in the total direct and triage pathway.

Probabilities to start a hearing aid trial, to have a follow-up and to have a successful hearing aid fitting were derived from a multi-centre non-controlled prospective evaluation study examining the shared care pathway versus the current pathway (AZOS working group, 2006). Participants, being persons with hearing complaints aged 50 years and older (valid $n=269$ ), first visited the hearing aid dispenser for a triage visit, where the dispenser examined whether the participant was in need of medical or specialized audiological care or not and hence should be defined as a patient or client respectively. All participants subsequently visited the ENT-specialist and AC to evaluate whether the dispenser had correctly defined them as a patient or a client. In cases where the participant was fitted with hearing aids he returned to the AC for a follow-up visit, to evaluate the quality of the hearing aid fitting. 
Because of the low incidence of pathology the study population of the prospective study was too small to obtain valid estimates of sensitivity and specificity of the private dispenser. Based on expert opinion we expected the probability of a private dispenser to refer patients to an ENT-specialist (sensitivity) to be at least as good as chance (0.5) and at maximum perfect (1), with a mean value of 0.75 . The probability of a private dispenser not to refer clients to an ENT-specialist (specificity) was estimated to be between 0 and 1, with a mean value of 0.5 . Hearing aid users are possibly more aware of their hearing problems and are more likely to seek help when their symptoms worsen. We therefore estimated the probability of undetected pathology to be detected to be higher for hearing aid users $(0.2)$ than for non-users (0.1).

Quality of the hearing aid fitting was based on a multi criteria evaluation instrument, of which details can be found elsewhere (AZOS working group, 2006). The probability to have a good quality hearing aid fitting under responsibility of the dispenser was 0.85 , as found in the prospective study. We assumed that for undetected acoustic neuroma, undetected otosclerosis and undetected psychosocial problems the hearing aid fitting would always be of poor quality, as these pathologies should have been considered in the hearing aid fitting. We estimated the probability to become an ex-user to be $50 \%$ higher for hearing aid users with a poor quality hearing aid fitting (0.18) than with a good quality hearing aid fitting (0.12).

We used age-specific mortality rates for five year age groups (Central Bureau for Statistics, 2007).

\section{Effects}

Health-related quality of life was used as outcome measure in the model. Health-related quality of life was considered as single index utility, on a scale from 0 to 1 , where 0 represents death and 1 represents perfect health. The use of utility scores allows the calculation of Quality Adjusted Life Years (QALYs) and cost per QALY ratios. Utility scores were derived with the HUI3, a measure responsive to change in health state utility after hearing aid fitting (Barton et al., 2004; Grutters et al., 2007a). For persons without pathology, we used the utility scores of participants in the prospective study, with and without hearing aid fitting (Table 7.2). We assumed ex hearing aid users to have the same level of utility as persons without hearing aid fitting. Based on expert opinion we derived HUI3 utility scores for persons with pathology. We estimated that persons with chronic otitis media and otosclerosis had more hearing complaints than persons without pathology, and that persons with chronic otitis media had more pain than persons with otoslerosis. Undetected psychosocial problems were associated with more problems on the emotional level. An acoustic neuroma was associated with more pain and more hearing loss than no pathology. We estimated that quality of the hearing aid fitting did not influence utility, as we did not find a relation between the two in the prospective study. 


\section{Costs}

All costs were in Euro and price indices were used to convert costs to a 2006 price level (Central Bureau for Statistics, 2007). Costs were estimated for health states as well as transitions. Wherever possible, costs were based on the Dutch manual for cost research (Oostenbrink et al., 2004). Alternatively, hospital prices were used. Productivity costs were not included because they were not expected to be relevant in a population with hearing complaints aged 50 years and older (Joore et al., 2003b).

Costs of the triage and follow-up visit at the private dispenser were calculated using a micro-costing approach. We repeatedly $(n=9)$ measured the exact time that was needed to perform both the triage and the follow-up visit, at three different dispensers participating in the study. This resulted in a mean duration and standard error, which was multiplied by the average labor costs of a private dispenser, including rent, depreciation of equipment, energy, taxation and maintenance. All costs are listed in Table 7.2. 
Table 7.2 List of input parameters

\begin{tabular}{|c|c|c|c|c|}
\hline Input parameters & $\begin{array}{r}\text { Estimated } \\
\text { value }\end{array}$ & SE & Distribution & Source \\
\hline \multicolumn{5}{|l|}{ Transition probabilities } \\
\hline \multicolumn{5}{|l|}{ Incidence } \\
\hline Acoustic neuroma & 0.0001 & 0.0001 & dirichlet & 1 \\
\hline Chronic otitis media & 0.0013 & 0.0003 & dirichlet & 2 \\
\hline Otosclerosis & 0.0001 & 0.0001 & dirichlet & 2, EO \\
\hline Psychosocial problems & 0.0175 & 0.0009 & dirichlet & 3 \\
\hline \multicolumn{5}{|l|}{ Help-seeking for hearing complaints } \\
\hline Seeking help & 0.16 & fixed & & 4 \\
\hline Increase in help-seeking in total direct and triage pathways & 0.10 & * & beta & $\mathrm{EO}$ \\
\hline \multicolumn{5}{|l|}{ Process of hearing aid (HA) fitting for patients with pathology } \\
\hline $\begin{array}{l}\text { Having HA with acoustic neuroma, otitis media, } \\
\text { otosclerosis }\end{array}$ & 0.75 & 0.03 & beta & $\mathrm{EO}$ \\
\hline Having HA with psychosocial problems & 0.90 & 0.02 & beta & $\mathrm{EO}$ \\
\hline Getting treatment for acoustic neuroma & 0.20 & 0.06 & beta & $\mathrm{EO}$ \\
\hline Being successfully treated for otitis media first 4 years & 0.80 & 0.06 & beta & $\mathrm{EO}$ \\
\hline Being successfully treated for otitis media after 4 years & 1 & fixed & & $\mathrm{EO}$ \\
\hline Being successfully treated for psychosocial problems & 0.50 & 0.29 & beta & $\mathrm{EO}$ \\
\hline \multicolumn{5}{|l|}{ Process of hearing aid (HA) fitting for persons without pathology } \\
\hline GP to refer to ENT-specialist & 0.69 & fixed & & 5 \\
\hline Starting hearing aid fitting & 0.58 & 0.03 & beta & OS \\
\hline Having a follow-up without pathology & 0.90 & 0.02 & beta & OS \\
\hline Having a hearing aid after follow-up & 0.98 & 0.01 & beta & OS \\
\hline \multicolumn{5}{|l|}{ Sensitivity } \\
\hline Dispenser to refer pathology to ENT-specialist & 0.75 & 0.14 & beta & $\mathrm{EO}$ \\
\hline \multicolumn{5}{|l|}{ Specificity } \\
\hline Dispenser not referring no pathology to ENT & 0.50 & 0.29 & beta & $\mathrm{EO}$ \\
\hline \multicolumn{5}{|l|}{ Detecting undetected pathology } \\
\hline Detecting undetected pathology in HA users & 0.20 & & uniform 0-1 & $\mathrm{EO}$ \\
\hline Detecting undetected pathology in non HA users & 0.10 & & uniform $0-1$ & $\mathrm{EO}$ \\
\hline \multicolumn{5}{|l|}{ Quality hearing aid fitting } \\
\hline Having a good quality HA fitting after follow-up dispenser & 0.85 & 0.07 & beta & OS \\
\hline \multicolumn{5}{|l|}{ Becoming ex user } \\
\hline Becoming ex user with poor quality HA fitting & 0.18 & 0.01 & beta & 6, EO \\
\hline Becoming ex user with good quality HA fitting & 0.12 & 0.01 & beta & 6, EO \\
\hline
\end{tabular}

\section{Utility scores}

No pathology, no hearing aid / ex user

No pathology, hearing aid user

Acoustic neuroma detected, no hearing aid

Acoustic neuroma detected, hearing aid user

Acoustic neuroma detected, after treatment

$\begin{array}{llll}0.63 & 0.02 & \text { beta } & \text { OS } \\ 0.70 & 0.02 & \text { beta } & \text { OS } \\ 0.42 & 0.02 & \text { beta } & \text { EO } \\ 0.51 & 0.02 & \text { beta } & \text { EO } \\ 0.23 & 0.02 & \text { beta } & \text { EO }\end{array}$


Table 7.2 List of input parameters (continued)

\begin{tabular}{|c|c|c|c|c|}
\hline Input parameters & $\begin{array}{r}\text { Estimated } \\
\text { value }\end{array}$ & SE & Distribution & Source \\
\hline Acoustic neuroma undetected, no hearing aid & 0.35 & 0.02 & beta & $\mathrm{EO}$ \\
\hline Acoustic neuroma undetected, hearing aid user & 0.41 & 0.02 & beta & $\mathrm{EO}$ \\
\hline Otitis media detected & 0.52 & 0.02 & beta & $\mathrm{EO}$ \\
\hline Otitis media undetected, no hearing aid & 0.52 & 0.02 & beta & $\mathrm{EO}$ \\
\hline Otitis media undetected, hearing aid user & 0.56 & 0.02 & beta & $\mathrm{EO}$ \\
\hline Otosclerosis detected, no hearing aid & 0.58 & 0.02 & beta & $\mathrm{EO}$ \\
\hline Otosclerosis detected, hearing aid user & 0.62 & 0.02 & beta & EO \\
\hline Otosclerosis undetected, no hearing aid & 0.31 & 0.02 & beta & $\mathrm{EO}$ \\
\hline Otosclerosis undetected, hearing aid user & 0.45 & 0.02 & beta & $\mathrm{EO}$ \\
\hline Psychosocial problems detected, no hearing aid & 0.63 & 0.02 & beta & $\mathrm{EO}$ \\
\hline Psychosocial problems detected, hearing aid user & 0.70 & 0.02 & beta & $\mathrm{EO}$ \\
\hline Psychosocial problems undetected, no hearing aid & 0.54 & 0.02 & beta & $\mathrm{EO}$ \\
\hline Psychosocial problems undetected, hearing aid user & 0.59 & 0.02 & beta & $\mathrm{EO}$ \\
\hline \multicolumn{5}{|l|}{ Costs } \\
\hline $\begin{array}{l}\text { Ratio of surgery versus radiotherapy as treatment for } \\
\text { acoustic neuroma }\end{array}$ & 0.50 & 0.03 & beta & $\mathrm{EO}$ \\
\hline $\begin{array}{l}\text { Probability of } \mathrm{CT} \text { scan in diagnosing chronic otitis media } \\
\text { and otosclerosis }\end{array}$ & 0.50 & 0.29 & beta & $\mathrm{EO}$ \\
\hline $\begin{array}{l}\text { Costs of extra procedures at ENT/AC for no pathology } \\
\text { when referred by GP or when referred by dispenser if } \\
\text { specificity }<0.75^{* *}\end{array}$ & 68 & 10 & gamma & $7, \mathrm{OS}$ \\
\hline $\begin{array}{l}\text { Costs of extra procedures at ENT/AC for no pathology } \\
\text { when referred by dispenser and specificity } \geq 0.75^{* *}\end{array}$ & 138 & 24 & gamma & $7, \mathrm{OS}$ \\
\hline Annual costs of batteries and maintenance, per hearing aid & 52 & fixed & & PC \\
\hline Annual cost of hearing aid repair & 28 & fixed & & PC \\
\hline \multicolumn{5}{|l|}{ Resource use } \\
\hline Number of hearing aids fitted per person & 1.78 & 0.04 & beta & OS \\
\hline MRI in acoustic neuroma wait \& scan state & 1 & fixed & & $\mathrm{EO}$ \\
\hline MRI after radiotherapy treatment for acoustic neuroma & 1 & fixed & & $\mathrm{EO}$ \\
\hline ENT consults in acoustic neuroma wait \& scan state & 1.50 & 0.29 & beta & $\mathrm{EO}$ \\
\hline ENT consults in acoustic neuroma after treatment state & 2.00 & 0.50 & gamma & $\mathrm{EO}$ \\
\hline ENT consults in detected otitis media state & 4.00 & 1.00 & gamma & $\mathrm{EO}$ \\
\hline Treatments (tympanoplasty) in detected otitis media state & 1 & fixed & & $\mathrm{EO}$ \\
\hline Combined ENT/AC consults in detected otosclerosis state & 1.00 & 0.25 & gamma & $\mathrm{EO}$ \\
\hline $\begin{array}{l}\text { Clinical audiologist / AC consults in detected psychosocial } \\
\text { problems state }\end{array}$ & 4.00 & 1.00 & gamma & $\mathrm{EO}$ \\
\hline Initial assessments at dispenser & 1 & fixed & & $\mathrm{EO}$ \\
\hline GP visits when seeking help with GP & 1 & fixed & & $\mathrm{EO}$ \\
\hline Combined ENT/AC consults when referred to ENT/AC & 1 & fixed & & $\mathrm{EO}$ \\
\hline $\begin{array}{l}\text { Combined ENT/AC follow-up visits to evaluate hearing aid } \\
\text { fitting }\end{array}$ & 1.22 & 0.04 & beta & OS \\
\hline Follow-up visits at dispenser & 1 & fixed & & $\mathrm{EO}$ \\
\hline BERA when diagnosing acoustic neuroma & 1 & fixed & & $\mathrm{EO}$ \\
\hline MRI when diagnosing acoustic neuroma & 1 & fixed & & EO \\
\hline
\end{tabular}


Table 7.2 List of input parameters (continued)

\begin{tabular}{|c|c|c|c|c|}
\hline Input parameters & $\begin{array}{r}\text { stimated } \\
\text { value }\end{array}$ & SE & Distribution & Source \\
\hline ENT consults when diagnosing acoustic neuroma & 2 & fixed & & $\mathrm{EO}$ \\
\hline Tympanometry when diagnosing chronic otitis media & 1 & fixed & & EO \\
\hline Tympanometry when diagnosing otosclerosis & 1 & fixed & & $\mathrm{EO}$ \\
\hline Stapedectomy when having otosclerosis & 1.80 & $* * *$ & beta & $\mathrm{EO}$ \\
\hline $\begin{array}{l}\text { Clinical audiologist consults when diagnosing } \\
\text { psychosocial problems }\end{array}$ & 1 & fixed & & \\
\hline ENT consults related to treatment for acoustic neuroma & 1 & fixed & & $\mathrm{EO}$ \\
\hline MRI related to treatment for acoustic neuroma & 1 & fixed & & $\mathrm{EO}$ \\
\hline \multicolumn{5}{|l|}{ Unit costs $(€)$} \\
\hline Hearing aid fitting, per hearing aid & 332 & fixed & & $8, \mathrm{OS}$ \\
\hline Hearing aid, without fitting, per hearing aid & 519 & fixed & & $8, \mathrm{OS}$ \\
\hline GP visit & 21 & fixed & & 7 \\
\hline Initial assessment at dispenser & 42 & 3 & gamma & OS \\
\hline ENT consult & 65 & fixed & & 7 \\
\hline Consult with clinical audiologist / audiological centre (AC) & 107 & fixed & & $\mathrm{HC}$ \\
\hline Combined ENT/AC consult & 162 & fixed & & 7 \\
\hline Combined ENT/AC follow-up to evaluate hearing aid fitting & 72 & fixed & & 7 \\
\hline Follow-up at dispenser & 41 & 3 & gamma & OS \\
\hline MRI & 251 & fixed & & 7 \\
\hline BERA & 338 & fixed & & $\mathrm{HC}$ \\
\hline CT scan & 276 & fixed & & 7 \\
\hline Tympanometry & 64 & fixed & & $\mathrm{HC}$ \\
\hline Stapedectomy & 1352 & fixed & & 7 \\
\hline Surgery as treatment for acoustic neuroma & 4120 & fixed & & 7 \\
\hline Radiotherapy as treatment for acoustic neuroma & 9958 & fixed & & 7 \\
\hline Treatment for chronic otitis media (tympanoplasty) & 1640 & fixed & & 7 \\
\hline
\end{tabular}

* A skewed distribution between 0 and 1 was fitted with a mean of 0.1 , and $\alpha=1, \beta=9$.

** For the calculation of average costs of procedures at ENT for persons without pathology we assumed the average costs to be higher when specificity was higher. For specificity higher than 0.75 we assumed that the dispenser would refer the subpopulation (25\%) who received further diagnostics in the observational study and thus had higher costs.

*** A skewed distribution between 0 and 1 was fitted with a mean of 0.2 , and $\alpha=1, \beta=4$, as the probability that the stapedectomy was not performed on both ears, and was then subtracted from 2 to obtain the number of stapedectomies.

Abbreviations: $\mathrm{OS}=$ observational study; $\mathrm{EO}=$ expert opinion; $\mathrm{PC}=$ personal communication with $\mathrm{H}$. Streukens; $\mathrm{HC}=$ hospital costs; $\mathrm{HA}=$ hearing aid; $\mathrm{SE}=$ standard error; $\mathrm{AC}=$ audiological centre References: 1 (Propp et al., 2006); 2 (Cummings, 2005); 3 Duijvestijn et al, unpublished data; 4 (Chorus et al., 1995); 5 (Duijvestijn et al., 1999); 6 (Joore et al., 2003b) 7 (Oostenbrink et al., 2004); 8 (Ziekenfondsraad, 1994) 


\section{Analysis}

We compared the cost-effectiveness of the total direct pathway, the triage pathway, the follow-up pathway and the current pathway. Incremental cost-effectiveness ratios (ICERs) were calculated, dividing the incremental costs by the incremental QALYs. ICERs were calculated by comparing each pathway with the next most effective pathway. Whether a pathway is deemed efficient depends on how much society is willing to pay for a gain in effect, which is referred to as the ceiling ratio. In the Netherlands an informal ceiling ratio of $€ 80000$ per QALY exists (Council for Public Health and Health Care, 2006). This is however a maximum ceiling ratio which applies when there is a high burden of disease, and is therefore not likely to apply to shared care in hearing aid provision. The National Institute for Health and Clinical Excellence in the United Kingdom uses a ceiling ratio between $£ 20000$ and $£ 30000$ per QALY (Buxton, 2006), which is roughly $€ 40000$.

Uncertainty surrounding the ICERs was handled probabilistically. This means that we assigned distributions to the model parameters, to reflect the second-order uncertainty in the estimation of that parameter (Weinstein, 2006). Measures of variance were retrieved from the prospective study, the patient cohort or published literature and, if no other source was available, from expert opinion. See Table 7.2 for the assigned distributions. Parameter values were drawn at random from the assigned distributions, using Monte Carlo simulation with 10000 iterations. To illustrate the results of the simulation, costeffectiveness acceptability curves (CEACs) were calculated (Fenwick et al., 2001; Van Hout et al., 1994). For different ceiling ratios, the net monetary benefit was calculated for each pathway by subtracting the costs from the effects, multiplied by the ceiling ratio. CEACs show the probability that a pathway has the highest net monetary benefit, and thus is deemed cost-effective, given different ceiling ratios.

In the base case analysis, age was set at 70 years, as this was the median age of participants in the prospective study, and was estimated to be the median age for persons seeking help for their hearing complaints. In a subgroup analysis, we varied age to 50 and 85 years.

As uncertainty exists, there is always a chance that the 'wrong' decision will be made (Briggs et al., 2006). The expected value of perfect information (EVPI) is a measure of the worth of acquiring additional information, through further research (Claxton \& Posnett, 1996). The EVPI is the expected value of obtaining perfect knowledge of the 'true' values of all parameters. We calculated the total EVPI by subtracting the net monetary benefit of the pathway we would choose under conditions of uncertainty, from the net monetary benefit of the optimal decision we would make if we knew the 'true' parameter values. Finally, we calculated the EVPI for (groups of) parameters, for a ceiling ratio of $€ 40000$ per QALY, to examine for which parameters further research is the most valuable (Felli \& Hazen, 1998). We calculated this partial EVPI by subtracting the net monetary benefit of the pathway we would choose under conditions of uncertainty for a given parameter (group), from the net monetary benefit of the optimal decision we would make if we knew the 'true' value of that parameter (group) (Groot Koerkamp et al., 2006). 


\section{Results}

\section{Base case analysis}

The current pathway yielded the most QALYs (on average 7.659; Table 7.3), followed by the follow-up pathway. The follow-up pathway was associated with a slight QALY loss of 0.005 and cost savings of $€ 40$, resulting in a mean ICER of $€ 8381$ saved per QALY lost. As both the triage pathway and the total direct pathway were somewhat more expensive than the follow-up pathway and associated with a loss in QALYs, these pathways were judged inferior.

Table 7.3 Base case results and one-way sensitivity analyses

\begin{tabular}{|c|c|c|c|c|}
\hline Pathway & $\begin{array}{r}\text { Expected costs in } € \\
(95 \% \mathrm{CI})\end{array}$ & $\begin{array}{r}\text { Expected QALYs } \\
(95 \% \mathrm{CI})\end{array}$ & \multicolumn{2}{|c|}{$\begin{array}{r}\text { (€ saved per } \\
\text { QALY lost) }\end{array}$} \\
\hline \multicolumn{5}{|c|}{70 years (base case analysis) } \\
\hline Current pathway & $1486(1345-1804)$ & $7.659(7.34-7.96)$ & & \\
\hline Follow-up pathway & $1447(1314-1766)$ & $7.654(7.33-7.96)$ & Current pathway & 8381 \\
\hline Triage pathway & $1491(1283-1815)$ & $7.646(7.34-7.95)$ & Follow-up pathway & inferior \\
\hline Total direct pathway & $1449(1250-1772)$ & $7.641(7.34-7.95)$ & Follow-up pathway & inferior \\
\hline \multicolumn{5}{|l|}{50 years } \\
\hline Current pathway & $2359(2089-3161)$ & $15.120(14.48-15.72)$ & & \\
\hline Follow-up pathway & $2286(2035-3090)$ & $15.109(14.47-15.71)$ & Current pathway & 6453 \\
\hline Triage pathway & $2304(2013-3127)$ & $15.042(14.48-15.70)$ & Follow-up pathway & inferior \\
\hline Total direct pathway & $2228(1955-3053)$ & $15.031(14.46-15.69)$ & Follow-up pathway & 743 \\
\hline \multicolumn{5}{|l|}{85 years } \\
\hline Current pathway & $664(611-748)$ & $3.104(2.97-3.23)$ & & \\
\hline Follow-up pathway & $651(599-734)$ & $3.103(2.97-3.23)$ & Current pathway & 13148 \\
\hline Triage pathway & $671(567-774)$ & $3.101(2.97-3.23)$ & Follow-up pathway & inferior \\
\hline Total direct pathway & $656(554-757)$ & $3.100(2.97-3.22)$ & Follow-up pathway & inferior \\
\hline
\end{tabular}

95\% Confidence Intervals $(\mathrm{Cl})$ are based on probabilistic analysis

In Figure 7.2 the cost-effectiveness acceptability curves show that considerable uncertainty surrounds the decision which pathway is deemed cost-effective. Given a ceiling ratio of $€ 40$ 000, the current pathway had the highest probability of being cost-effective (54\%), followed by the triage pathway $(40 \%)$, the follow-up pathway $(4 \%)$ and the total direct pathway $(2 \%)$. 


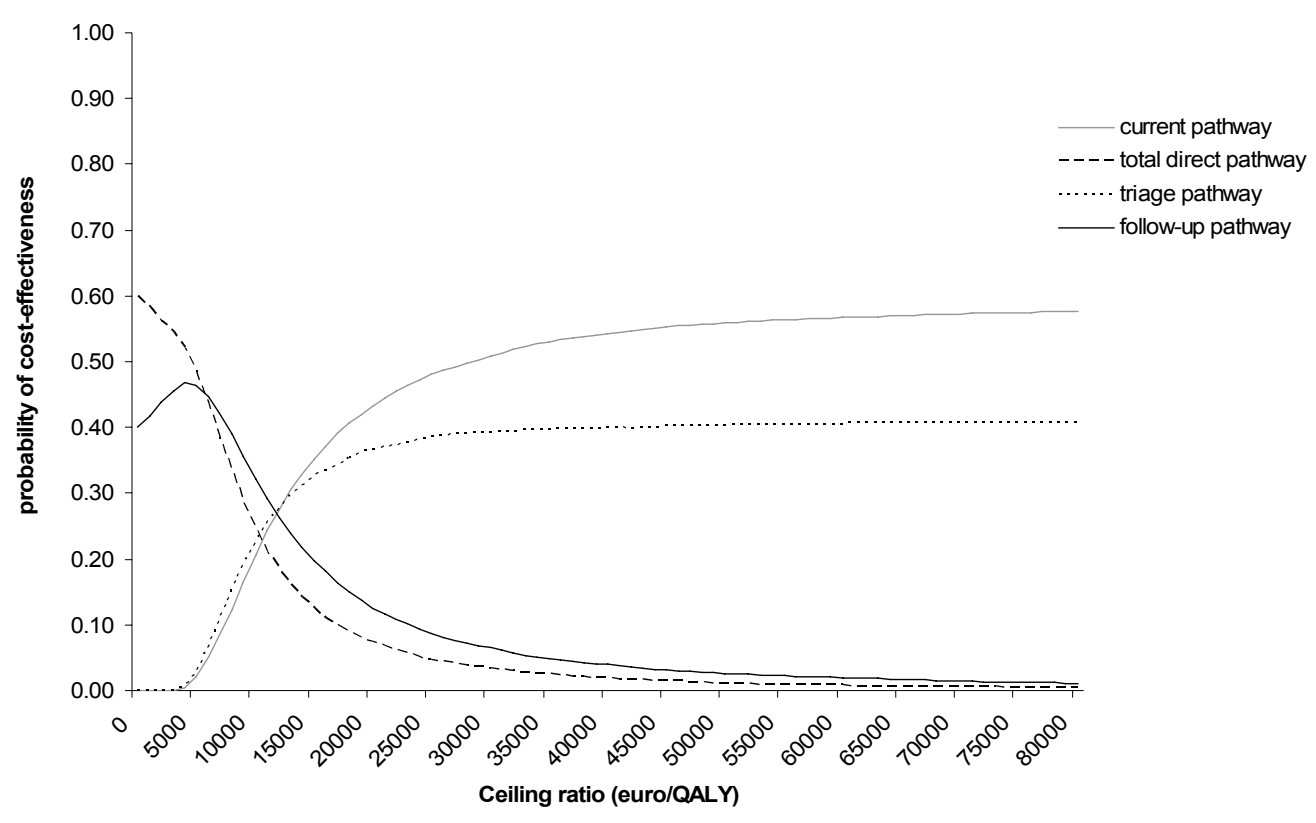

Figure 7.2 Cost-effectiveness acceptability curves for the four pathways

\section{Subgroup analysis for age}

To examine whether age influenced the results of the cost-effectiveness analysis, we varied age to 50 and 85 years (Table 7.3). For persons aged 50, the current pathway yielded the most QALYs (15.120), followed by the follow-up pathway. The follow-up pathway resulted in a QALY loss of 0.011, cost savings of $€ 73$, and hence an ICER of $€ 6453$ saved per QALY lost. The triage pathway was more expensive and less effective than the follow-up pathway, so this pathway was inferior. The total direct pathway was less expensive and less effective than the follow-up pathway, resulting in an ICER of $€ 641$ saved per QALY lost. Given a ceiling ratio of $€ 40000$ the current pathway, follow-up pathway, triage pathway and total direct pathway had a probability of being cost-effective of $61 \%, 3 \%, 35 \%$ and $1 \%$ respectively.

For persons aged 85, the current pathway also yielded the most QALYs (3.104), followed by the follow-up pathway. The follow-up pathway resulted in cost savings of $€ 14$ and a QALY loss of 0.001, and hence an ICER of $€ 13148$ saved per QALY lost. Both the total direct pathway and the triage pathway were more expensive and less effective than the follow-up pathway, and thus inferior. The current, follow-up, triage and total direct pathway had a $46 \%, 10 \%, 38 \%$ and $6 \%$ probability of being cost-effective respectively, given a ceiling ratio of $€ 40000$. 


\section{Expected value of perfect information}

For the base case analysis the uncertainty surrounding the decision whether or not to implement shared care resulted in an EVPI of $€ 193$ per person, given a ceiling ratio of $€ 40$ 000. Implementing shared care affects all persons with hearing complaints of 50 years and older without a hearing aid, being a total of 1142835 persons in the Netherlands in the next 10 years (Central Bureau for Statistics, 2007). This makes the population EVPI $€ 110$ million, meaning that perfect information on this topic is worth $€ 110$ million. The population EVPI for different ceiling ratios is presented in Figure 7.3.

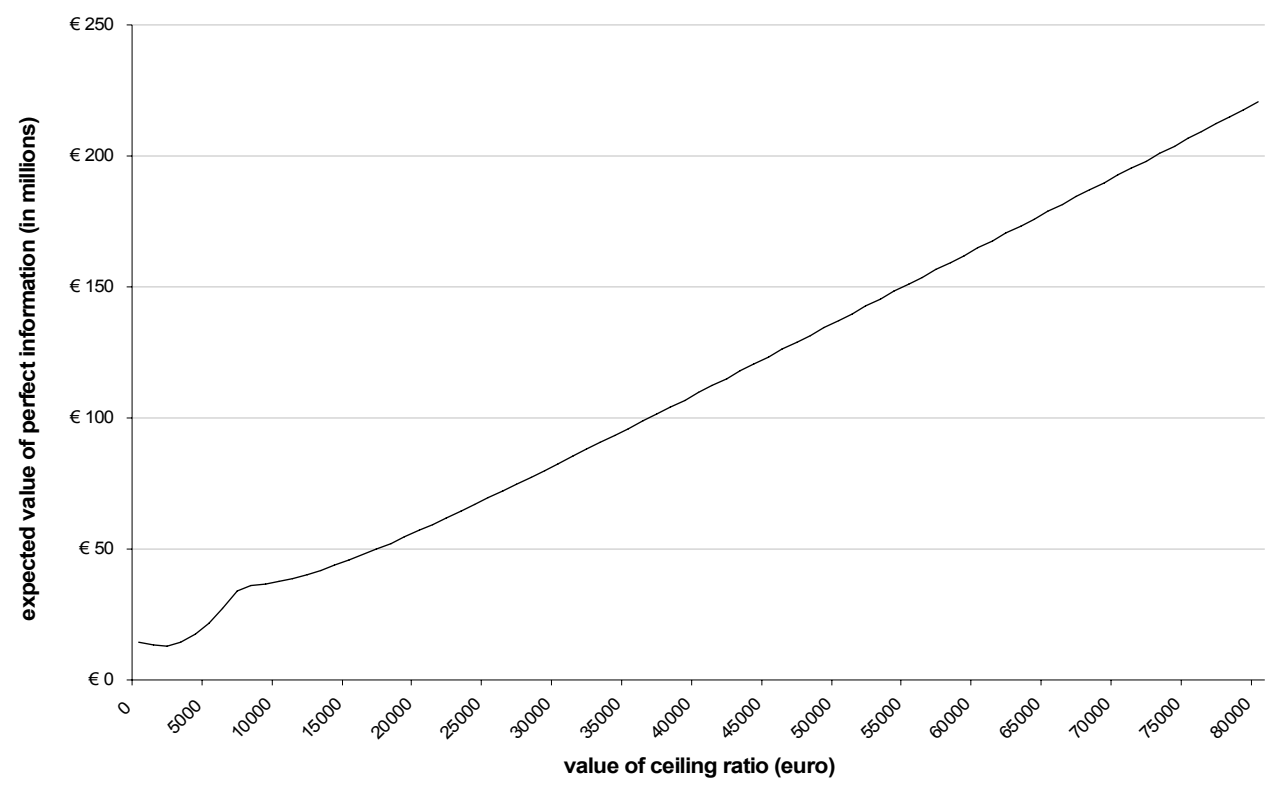

Figure 7.3 Population expected value of perfect information curve

Additionally, the EVPI for parameters was calculated. The parameter most valuable for further research was whether persons with hearing complaints would seek help sooner when they can do so directly with the private dispenser (Figure 7.4). Another valuable parameter for further research was the probability that the private dispenser refers patients to the ENT-specialist (sensitivity), followed by the probability that undetected pathology will be detected, and the utility scores. 


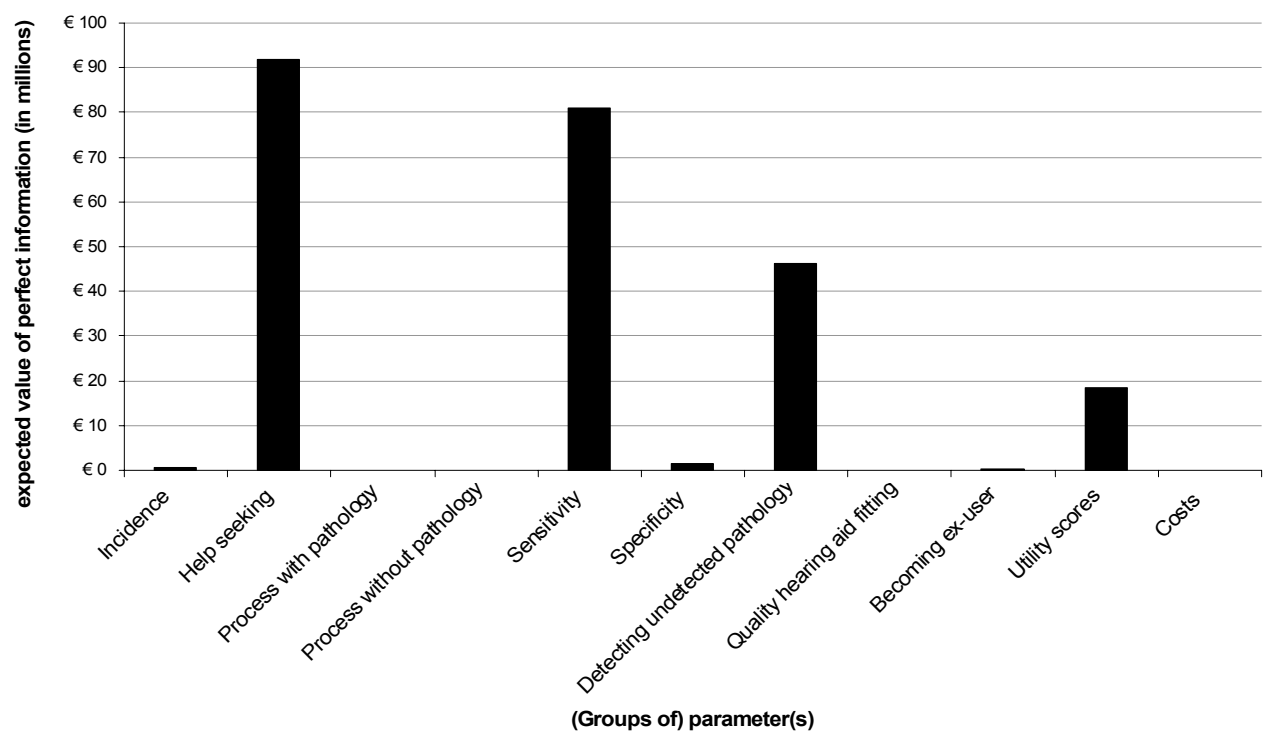

Figure 7.4 Expected value of perfect information for (groups of) parameters

\section{Discussion}

Using decision-analytic modeling, we found that the differences in costs and effects between the pathways were small. The current pathway was slightly more effective than the shared care pathways. The follow-up pathway was both somewhat less expensive and less effective than the current pathway, resulting in an ICER of $€ 8381$ saved per QALY lost. The results indicate that none of the alternative shared care pathways would be deemed cost-effective, especially the total direct pathway and the triage pathway as they were more expensive and less effective than the follow-up pathway. However, the ICERs are surrounded by considerable uncertainty. Given a ceiling ratio of $€ 40000$, the current pathway had the highest probability of being cost-effective (54\%), followed by the triage pathway $(40 \%)$, the follow-up pathway $(4 \%)$ and the total direct pathway (2\%). Subgroup analyses showed that shared care became more favorable with increasing age, although the uncertainty remained high. The value of totally eliminating uncertainty was $€ 110$ million in the base case analysis, for a ceiling ratio of $€ 40000$.

Given the present results, implementing any form of shared care in hearing aid provision at this stage is not recommended. Further research is needed to reduce the uncertainty. Although some uncertainty will always remain and hence perfect information is unfeasible, the population EVPI showed that the value of further research on this topic greatly outweighs its costs. One of the most valuable topics for further research was the sensitivity of the private dispenser to recognize pathology. More research is needed on whether the dispensers are capable of identifying the subgroup of persons who require medical care. 
Another important topic is the probability that a person with hearing complaints will seek help for his complaints. The results from a survey indicated that a quarter of the participants considered the visits to GP, ENT-specialist and AC as a barrier for hearing aid fitting (Grutters et al., 2007b). However, we are unsure whether more persons will actually seek help in the shared care pathway, and how much more. Probably, shared care will only result in health gain when barriers are lowered and more hearing-impaired persons are adequately fitted with hearing aids. Otherwise, there will be no health benefit but only a health loss for a small cost reduction. It is doubtful whether the latter would be deemed desirable by society. More research is therefore needed on whether shared care will actually lower barriers to seek help for hearing complaints. A possible disadvantage of shared care is that the prescriber of the hearing aid financially benefits from selling hearing aids. This may result in over-provision of hearing aids, and a higher probability of becoming an ex user. Although over-provision was beyond the scope of the present paper, it should be considered if in a later stage shared care is found to be cost-effective and may be implemented, especially when it is found that shared care actually lowers barriers to help-seeking.

The current paper demonstrates the use and usefulness of decision-analytic modeling in health services research. Some years ago Hadley presented two major issues in health services research: 1) there is not enough data to answer questions with enough precision for people to use in making decisions, and 2) collecting data is expensive, and health services research does not have enough budget to provide helpful answers (Hadley, 2000). Decision-analytic modeling can assist in both these issues. First, all available evidence can be synthesized in a model to guide decision-making. Next, value of information analyses can help to allocate research budgets in the most efficient way. This will obviously not answer all questions that exist in health services research, but will at least help to choose the most valuable topics for future research.

There are a number of limitations of the present study that need to be addressed. First, we only incorporated four types of pathology, while also other types may affect hearing and may be missed by dispensers. However, modeling is always a simplification of a complex reality, and we feel that we included the most important types of pathology in this study, as they most frequently occur in this population and are most likely to be missed by dispensers. Although hearing loss can result from Lyme disease, we could not find a strong association between Lyme disease and hearing loss in the literature (Cummings, 2005; Gagnebin \& Maire, 2002). We therefore left Lyme disease out of the model, although we are aware that it is, and will probably increase to be, a possible cause of hearing loss, due to the increasing number of ticks infected with Borrelia burgdorferi (Hofhuis et al., 2006). Another limitation may be that many uncertain parameters were included in the model, based on expert opinion or small study populations. However, a model should not be faulted because existing data fall short of ideal standards of scientific rigor (Weinstein et al., 2003). That is, decisions will be made, so it is better to inform the decision with the available evidence than without any evidence at all. Using decision-analytic modeling we were able to incorporate this uncertainty in the model, and results were shown with surrounding uncertainty. We did assume 'perfect implementation', though. Less than 
perfect implementation reduces efficiency, for example when help-seeking with the dispenser is possible but hearing-impaired persons would continue to seek help with their GP. It is unclear whether patients and professionals will change their behavior if shared care would be implemented. If shared care in hearing aid provision would be deemed efficient, it would therefore be interesting to examine the value of perfect information. This would allow for a trade-off between the costs and benefits of implementation strategies (Fenwick et al., 2007; Hoomans et al., 2007).

A final limitation is that decision-analytic modeling is rather computer intensive. Especially for the partial EVPI, many iterations need to be drawn. In the present study, we ran 200 Monte Carlo simulations by 200 draws for the parameter (group) of interest. When repeatedly running our partial EVPI analysis the ordering of which (groups of) parameters were most valuable for further research remained stable. Thus, we could accurately estimate which (groups of) parameters are more and less valuable for further research. One should however be aware that, to answer the question how valuable obtaining perfect information for each parameter (group) exactly is, many more iterations would be required.

\section{Conclusion}

We can conclude that, based on the available evidence, at this stage implementation of shared care in hearing aid provision, in any form, is not recommended. This contradicts the current situation in the Netherlands, where shared care models are implemented. Furthermore, the results show that it is worthwhile to perform additional research to reduce uncertainty surrounding the decision whether or not to implement shared care. Additional research on whether shared care will lower barriers to help-seeking and whether dispensers are capable of identifying the subgroup that requires medical care is most valuable. Overall, this study shows that decision-analytic modeling in an early stage of organizational innovation is a valuable tool to come to evidence-based decision making. 


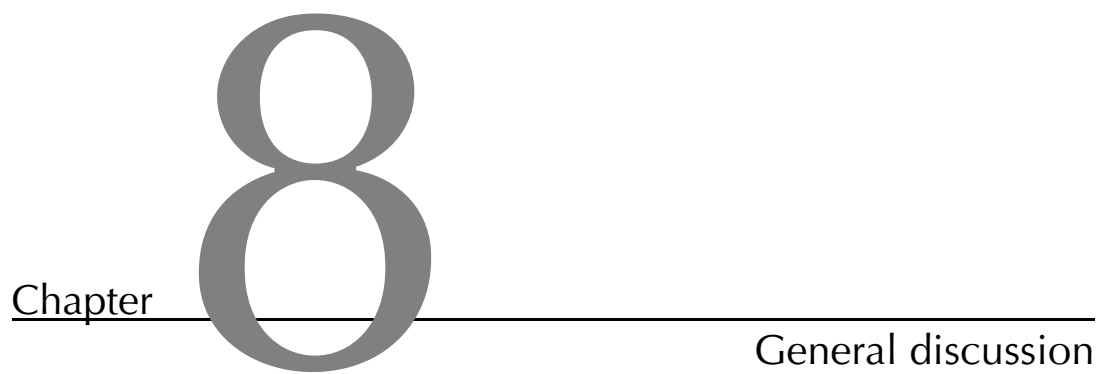



The objective of this thesis was to use health technology assessment to evaluate whether an organizational innovation, shared care in hearing aid provision, benefits the care for hearing-impaired persons. In this final chapter, the main findings regarding the research questions are summarized and discussed, followed by the methodological considerations. Subsequently, recommendations for policy and practice are described, and areas for future research are identified.

\section{Main findings}

The first research question addressed the expectations and potential barriers and facilitators towards shared care in hearing aid provision, of both patients and professionals. Hearing aid dispensers and general practitioners (GPs) had on average positive expectations towards the direct pathway, while the Ear Nose and Throat (ENT) specialists and clinical audiologists had on average negative expectations and were somewhat more reluctant towards implementation. This has also been observed in other health care settings where established professions develop strategies to protect their boundaries, while encroaching professions try to expand their work areas (Adams, 2004; Nancarrow \& Borthwick, 2005; Norris, 2001; Stevens et al., 2007). Despite these differences, most professionals either supported implementation of the shared care model, provided that a number of conditions are satisfied, or did not support implementation, unless roughly the same conditions are satisfied. These conditions were to arrange good communication between the professionals involved in the shared care model, to include adequate training for the hearing aid dispensers, to frequently evaluate the shared care model, to formulate clear criteria for referral, to define a clear division of tasks, to have a second opinion possibility, and to provide a complaints service. Patients evaluated the referral and shared care pathway equally and had confidence in the hearing aid dispenser. Many, especially older, participants however stated that they would still visit the GP and ENT-specialist, even when this would not be necessary for reimbursement, and found it important that the ENTspecialist or audiological centre (AC) evaluated their hearing aid fitting. This indicates that older persons are more attached to the opinion of the medical specialist.

The second research question focused on how to measure outcome in shared care in hearing aid provision. With regard to measuring utility, both the EuroQol 5D (EQ-5D) and the Health Utilities Index mark III (HUI3) seemed to be practical and valid. Only moderate to poor agreement was found between the measures. The HUI3 and the HUI mark II were more responsive to change after hearing aid fitting than the EQ-5D, and hence resulted in lower incremental cost-effectiveness ratios (ICERs) for hearing aid fitting. The results confirm other studies that found that different utility measures lead to different utility scores (Bosch \& Hunink, 2000; Brazier et al., 2004; Conner-Spady \& Suarez-Almazor, 2003; Espallargues et al., 2005; Feeny et al., 2004; Kaplan et al., 2005; Kopec \& Willison, 2003; Longworth \& Bryan, 2003; Marra et al., 2004; Marra et al., 2005; O'Brien et al., 2003; Oostenbrink et al., 2002; Petrou \& Hockley, 2005). With respect to the study population, 
our results confirm those of Barton et al. $(2005 ;$ 2004), who compared the EQ-5D and the HUI3 before and after hearing aid fitting in a British sample. The lower utility scores and higher responsiveness of the HUI3 as opposed to the EQ-5D were also found in a population with visual impairment (Espallargues et al., 2005).

In the contingent valuation study we found no statistically significant difference, and a strong association between the results of the open-ended and the payment scale question. The payment scale and the open-ended format both showed good criterion validity, though the open-ended willingness to pay (WTP) question showed a stronger association with the gold standard, the actual payment. Moreover, the open-ended WTP question showed better construct validity, since it was consistent with the theoretical construct of positive income elasticity. Although the open-ended format is often thought to be less valid than the payment scale format (Arrow, Solow, Portney, Leamer, Radner, \& Schuman, 1993; Donaldson, Thomas, \& Torgerson, 1997; Ryan, Scott, \& Donaldson, 2004), these results point towards the opposite. This may well be the result of the composition of our study population, as our study population consisted of hearing aid users who were familiar with payment for their hearing aid.

In the discrete choice experiment (DCE) we found that willingness to accept (WTA) exceeded willingness to pay, similar to what was found in contingent valuation studies on a variety of subjects (Horowitz \& McConnell, 2002; O'Brien et al., 2002). The disparity between WTA and WTP was of a magnitude to alter policy recommendations, as omitting the follow-up at the ENT-specialist would only be recommended when using a WTP format to elicit monetary valuations, and would not be recommended when using a WTA format for losers and a WTP format for gainers or only a WTA format.

From the discrete choice experiment it was found that Dutch hearing-impaired persons are receptive to transferring elements of hearing aid provision from the medical sector to private hearing aid dispensers. In general, participants preferred that hearing aids would be provided with an initial assessment at the hearing aid dispenser with the same accuracy as at the ENT-specialist, a duration of two months, and a follow-up at the ENT-specialist to evaluate the hearing aid fitting.

The third research question focused on whether shared care in hearing aid provision is efficient. We found that shared care in hearing aid provision results in both a cost reduction and a loss of quality adjusted life years (QALYs). Shared care becomes more favorable with increasing age, but for persons aged 85 there is still less than $€ 40000$ saved per QALY lost, which indicates that shared care is not cost-effective. However, considerable uncertainty surrounded the incremental cost-effectiveness ratios (ICERs). Given a ceiling ratio of $€ 40000$, there was a 54\% probability that the referral pathway would be deemed cost-effective. Of the alternative shared care models, the triage pathway had the highest probability of being cost-effective (40\%). It was found to be most valuable to perform additional research on whether shared care in hearing aid provision will lower barriers for help-seeking, and whether hearing aid dispensers are capable of distinguishing patients from clients. 
From the survey we found that persons aged 70 years and older, compared to persons under the age of 70, were more inclined to still visit the ENT-specialist or AC, even if this was not necessary for reimbursement of the hearing aid. This seems to contradict the findings of the discrete choice experiment, where older persons were found to have a stronger preference for the initial assessment at the dispenser as opposed to at the ENTspecialist. This contradiction may result from the fact that the youngest participant in the discrete choice experiment was 18, while the youngest participant in the survey was 50 years of age. Persons with hearing complaints who are under the age of 50 always need to see an ENT-specialist or AC, as their hearing loss is not only age-related and may be a symptom of a (treatable) medical condition. It is therefore not surprising that persons under the age of 50 stated that they wish to be examined by an ENT-specialist. Another possible explanation is the difference in question designs. In the survey respondents stated on a Likert scale whether they would still visit the ENT-specialist or AC, with no strings attached. On a Likert scale respondents make a choice that is not constrained, which may cause respondents to overstate their preferences (Ryan et al., 2001). In a discrete choice experiment the choice is constrained, as respondents make trade-offs between different attributes, which may well lead to different answers. It has been suggested that Likert scales are a useful technique for eliciting attitudes, but that conjoint analysis should be used to measure preferences (Phillips et al., 2002; Ryan et al., 2001).

We can combine the results of the DCE and the cost-effectiveness analysis to do a straightforward cost-benefit analysis. Monetary values for the three alternative pathways of shared care in hearing aid provision in the cost-effectiveness analysis can be estimated with the results of the DCE. For the total direct pathway, assuming a four months shorter duration, hearing-impaired persons requested compensation of at least $€ 437$, while it was found to save only $€ 37$ per person as opposed to the current pathway. For the triage pathway, assuming a two months shorter duration, minimum compensation of $€ 227$ was requested, while it was found to be $€ 5$ more expensive than the current pathway. For the follow-up pathway, also assuming a two months shorter duration, compensation of at least $€ 210$ was requested, while saving only $€ 39$ per person. As the resulting net benefits (benefits minus costs) are negative for all pathways, none should be implemented from a cost-benefit perspective. However, we need to emphasize that considerable uncertainty surrounded these costs. Besides, the cost-effectiveness analysis was based on the total population with hearing complaints, while the DCE included only the subpopulation of persons who seek help for their hearing complaints.

An interesting finding when comparing the results of the DCE and the cost-effectiveness analysis is that the triage pathway, with only introducing the triage at the dispenser but maintaining the evaluation of the hearing aid fitting at the ENT-specialist or AC, is the pathway that is preferred by the patients as well as the shared care model that is most likely to be cost-effective. 


\section{Methodological considerations}

Some methodological considerations arise from the use of health technology assessment in evaluating shared care in hearing aid provision.

First, it is questionable whether the Technology Assessment Iterative Loop (TAIL) is readily applicable to organizational innovations such as shared care in hearing aid provision. For example, organizational innovations need to be implemented in order to evaluate them. However, according to the loop a technology is not implemented until efficacy, community effectiveness and efficiency are proven. Also, according to the loop a trade-off between costs and consequences takes places only when efficacy and community effectiveness are proven. However, the use of decision-analytic modeling provides valuable information on the potential cost-effectiveness of the innovation, on how the innovation should be designed, and on which topics are most valuable for further research. Decision-analytical modeling therefore is a useful tool to examine efficiency in a very early stage, even before conclusive evidence on efficacy is available.

As organizational innovations require agreements and cooperation among health care professionals, randomized trials are often not appropriate or even possible (Winkens \& Klazinga, 2000). Pragmatic trials are probably more appropriate, as they allow for involvement of all parties and adjustments during the trial. However, this also involves some limitations. In our prospective study patients received usual care, with only an extra visit to the dispenser before the initial assessment at the ENT-specialist and AC. As patients were not randomized to separate arms and were not forced to a certain care pathway, a number of patients had their hearing aid fitted at dispensers not participating in the study, and were therefore lost to follow-up. Also, a number of patients had not finished their hearing aid trial before the end of the study. A less controlled study population results in a higher number of missing values and a more heterogenic study population. These limitations are among the risks of performing pragmatic research, but the advantage is that outcomes are probably more representative for the real world.

Also, in evaluating organizational innovation researchers tend to play a double role, as they both evaluate and implement the innovation. This has limitations, as they may be biased towards the innovation, or towards professionals, but also has advantages, as they are involved in the innovation process and can directly take up practice and quickly adjust to events. While the latter is not important in traditional health technology assessment (HTA), it may be when using HTA in organizational innovations. This again indicates that the traditional form of HTA may be less appropriate to organizational innovation than it is to for example pharmaceutical research. Possibly aspects of constructive technology assessment could be used to adapt the traditional HTA to organizational innovation. One of the key aspects of constructive technology assessment is to shift the focus of TA away from assessing fully developed technologies, and introduce anticipation of technology impacts at an early stage in the development (Rip et al., 1995). Also, a key aspect of constructive TA is to provide a continuous dialogue between 'potential or actual users of the technology, those who are affected by the technology and those who design and 
promote the technology'. Possibly these aspects of constructive TA may well apply to HTA in organizational innovation.

Modeling is always a simplification of a complex reality. Therefore, model assumptions need to be made. However, one should keep in mind that decision-analytic models are not intended to reveal scientific truth (Weinstein, 2006), but to guide decision-making. In modeling the efficiency of shared care in hearing aid provision, we assumed perfect implementation. It is however unclear whether patients and professionals will change their behavior if shared care in hearing aid provision would be implemented. Less than perfect implementation would reduce efficiency. Recently there has been growing interest in the value of investing in strategies to improve implementation, and whether one should invest in strategies to improve behavioral change. If shared care in hearing aid provision would be deemed efficient, it would be interesting to examine the value of perfect information, allowing for a trade-off between the costs and benefits of implementation strategies. Recently, frameworks were developed to calculate this value of perfect implementation (Fenwick et al., 2007; Hoomans et al., 2007). However, simply incorporating implementation parameters in the decision-analytic model may be a practicable alternative to separate frameworks in assessing the value of perfect implementation.

Finally, monetary valuation is able to encompass much more than the traditional QALY. That is, underlying preferences not directly related to health, such as accessibility or satisfaction, are incorporated in the monetary value, while they are not incorporated in the QALY. Aspects of accessibility and satisfaction are important in organizational innovations, and the trade-off between these outcomes and the clinical outcome is interesting. Therefore, monetary valuation and the cost-benefit analysis may be more appropriate than cost-utility analysis in this field. As respondents are often not familiar with the innovation, and several alternative designs of the innovation exist, DCE may well be more appropriate than contingent valuation. That is, in a DCE respondents are not directly asked to state an amount of money, and monetary values can be calculated for various configurations of levels. However, a lot of controversy remains on whether and how a cost attribute should be included in a DCE to calculate monetary values for use in a cost-benefit analysis (Hanley et al., 2003; McIntosh, 2006; Ratcliffe, 2000; Slothuus Skjoldborg \& Gyrd-Hansen, 2003). 


\section{Recommendations}

Shared care in hearing aid provision is not yet broadly based among professionals who are involved in the care for hearing-impaired persons. Especially ENT-specialists and clinical audiologists stated potential risks and were somewhat reluctant to implement a shared care model. Their concerns regarding safety and efficiency are supported by the costeffectiveness analysis, which showed that more evidence is needed on whether private dispensers are capable of detecting pathology. The professionals are united in the conditions that need to be fulfilled for a successful implementation of shared care in hearing aid provision. It is important that these conditions are addressed in the design or implementation process of shared care in hearing aid provision. Another important aspect is that all parties should be involved in the design and implementation process of the innovation. In small regions it possibly is less difficult to create a basis of trust among the professionals involved. When the professionals and hearing-impaired persons make agreements on a regional level, they will get to know each other better. As a result this might improve their communication and their confidence in the other professionals' capabilities. Implementation of shared care in hearing aid provision on a regional level is therefore recommended to best satisfy the stated conditions.

Regarding outcome measurement, it is clear that health state utility scores heavily depend on the measure that is used to elicit them. The use of QALYs is often recommended in economic evaluations as it allows for comparison across different interventions and health care sectors (Drummond et al., 2005). However, the impact of different utility measures on the ICER for hearing aid fitting is of a magnitude that can alter policy decisions. Hence, comparisons of QALYs across studies and interventions should be interpreted with caution. We recommend that the HUI3 is the instrument of first choice when measuring utility in a population with hearing complaints, but we emphasize the importance of a clear notion of what constitutes utility with regard to economic analyses in health care. This seems to be especially important for organizational innovations, where health is not always the most obvious maximand. From the comparison of different question formats in contingent valuation, we recommend that in future WTP studies on hearing aids the open-ended question is used to directly elicit WTP values. This recommendation may also apply to other study settings where respondents are familiar with costs or payments for the intervention under evaluation. When eliciting monetary values in a DCE we recommend that a normative decision is made on whether the cost attribute should be defined as a payment or a discount to elicit monetary values. Until this normative decision is made, we recommend to choose the most conservative format, which is a payment (WTP) if (the majority of) respondents are potential gainers, and a discount (WTA) if (the majority of) respondents are potential losers. However, this may affect the flexibility of discrete choice experiments to calculate monetary values for different configurations of interest within a cost-benefit analysis.

With regard to shared care in hearing aid provision, we do not recommend to implement shared care, in any form, just yet. As the value of further research on this topic greatly outweighs its costs, we recommend to first reduce some uncertainty, especially with regard 
to whether shared care in hearing aid provision will increase help-seeking among persons with hearing complaints, and whether dispensers are capable of detecting pathology. Subgroup analyses showed that shared care in hearing aid provision becomes more efficient with increasing age. This corresponds to the fact that in the DCE older persons had a stronger preference for the initial assessment at the hearing aid dispenser. However, as the results of the survey indicated that older persons were more attached to the opinion of the medical specialist, shared care should not just be recommended for a subgroup of older persons without taking into account their preferences.

Despite these recommendations, it is important to realize that in some regions of the Netherlands, a number of health insurance companies already permit a form of direct hearing aid provision by selected private dispensers. For example, some insurance companies and dispenser practices have made agreements that an evaluation of the hearing aid fitting by an ENT-specialist or AC is no longer needed for reimbursement. A few years ago policy makers in the Netherlands decided that the provision of medical aids should be deregulated. This would give insurance companies room for competition and would give patients room for choice by comparing the insurance companies. In the case of hearing aid provision, the insurance companies make use of the free rein they were given, by making agreements with hearing aid dispenser practices. It is however doubtful whether this benefits the care for hearing-impaired persons. The results of this thesis indicate that it is unlikely that the agreements that are currently being made are efficient, and hence market regulation seems to fail. However, agreements have been made and will probably not become undone. It is important that policy makers become aware of this consequence of the deregulation. They should attend to caution in the provision of hearing aids, and to quality standards with regard to existing and future agreements and careful evaluation of the agreements.

Private hearing aid dispensers are not a legally protected professional group in the Netherlands. This means that anyone can call himself a private dispenser. When dispensers are going to be included in the shared care pathway, it is highly recommended that they develop a code of conduct, which should be guaranteed by a supervisory board and a conciliation board. The Dutch register foundation of hearing aid dispensers (Stichting Audicienregister, StAr) has formulated quality standards and keeps a register of hearing aid dispensers that fulfill these standards. Also, since January 2007 the foundation provides hearing aid dispenser practices that work according to the standards with a quality mark. In addition, the Comité Européen de Normalisation (CEN) has recently decided to formulate European quality standards to the work area and professional practice of hearing aid dispensers. These initiatives are important for the private dispensers to become a serious partner and to gain responsibility in the process of hearing aid provision. Moreover, such initiatives are needed to guarantee the quality and safety of shared care models in hearing aid provision. 
In general, we believe that in the process of organizational innovations in health care, health technology assessment should occupy a more important place. It is rather surprising that the stringent rules for pharmaco-economic research do not apply to health services research. Each new drug needs conclusive evidence before it can be introduced to the market. In contrast, organizational innovations can be implemented without formal evidence, except with regard to tasks which are reserved to specific health care professionals by law. We believe that decision-making with regard to organizational innovation in health care should be based more on thorough evaluation by means of health technology assessment, thus facilitating evidence-based decision-making. This thesis provides a practical example of how this can be done. However, more work is needed to adapt health technology assessment to the field of organizational innovation. Possibly adapting some of the key issues of constructive technology assessment, such as a continuous dialogue between involved parties and assessment at an early stage, would make HTA more appropriate to organizational innovation.

In conclusion, we recommend that in organizational innovations HTA should be applied from a very early stage. Decision-analytic modeling can be used to integrate all available evidence to inform the design process and the topics most valuable for further research. Also, all parties involved in the innovation, patients as well as professionals, should be involved in a very early stage to understand the important issues in the field and to involve them in the design process. Involvement in the design of the innovation will probably enhance the chances that the parties support the innovation and that the innovation fits the needs of these parties. Hence, early involvement facilitates the will of the parties to change their behavior when the innovation is implemented. Finally, the evidence that is obtained from health technology assessment should be used in decision-making with regard to organizational innovation, as is already practice for pharmaceutical and other strictly medical innovations. In order to make organizational innovations such as shared care in hearing aid provision beneficial to the care for hearing-impaired persons, it is important that they are evaluated appropriately. 


\section{Areas for future research}

Regarding outcome measurement, we emphasized the importance of a clear notion of what constitutes utility in economic evaluations in health care. An important area for future research is whether societies wish to, or should, maximize life expectancy corrected for health-related quality of life from a more functional perspective as in the EQ-5D, or from a 'within-the-skin' perspective as in the HUI. Moreover, there is need for consensus with regard to the content of the evaluative space in economic evaluations in broader terms. Should QALYs remain the recommended outcome measure, or should we look broader than health outcomes alone, for example by using monetary values? The results from the contingent valuation study add to the limited evidence on the validity of direct willingness to pay measures to elicit monetary values. However, more evidence is needed on the validity of directly measuring willingness to pay and its elicitation formats. As patient preferences gain increasing attention in decision making (Bridges \& Jones, 2007; Ryan, 2004), and cost-benefit analyses may be more appropriate when evaluating organizational innovation, discrete choice experiments will probably become increasingly important in health services research. Discrete choice experimentation seems to be a feasible instrument to measure monetary values. However, more research is needed to address methodological issues regarding the inclusion of a cost attribute to elicit monetary valuations with a discrete choice experiment (Hanley et al., 2003; Mclntosh, 2006; Ratcliffe, 2000; Slothuus Skjoldborg \& Gyrd-Hansen, 2003). Specifically, more research is needed on why the disparity between WTA and WTP occurs in a discrete choice experiment, and on when to use a payment or a discount in the cost attribute.

With regard to the safety and efficiency of shared care in hearing aid provision, more research is needed on whether hearing aid dispensers are capable of distinguishing between patients and clients. But even when it is found to be safe, shared care in hearing aid provision may only benefit the care for hearing-impaired persons when barriers towards help-seeking are lowered and, as a result, more hearing-impaired persons are appropriately fitted with hearing aids. Otherwise, there will be no health benefit but only a health loss for a small cost reduction. It is doubtful whether the latter would be deemed desirable by society. More research is therefore needed on whether shared care in hearing aid provision will actually cause persons to seek help for their hearing complaints sooner. 

Bibliography 

Abdelkader M, McEwan M and Cooke L (2004). Prospective evaluation of the value of direct referral hearing aid clinic in management of young patients with bilateral hearing loss. Clinical Otolaryngology and Allied Sciences 29(3):206-9.

Adams TL (2004). Inter-professional conflict and professionalization: dentistry and dental hygiene in Ontario. Social Science \& Medicine 58(11):2243-52.

Anell A and Norinder A (2000). Health outcome measures used in cost-effectiveness studies: a review of original articles published between 1986 and 1996. Health Policy 51(2):87-99.

Appollonio I, Carabellese C, Frattola L, and Trabucchi M (1996). Effects of sensory aids on the quality of life and mortality of elderly people: a multivariate analysis. Age \& Ageing 25(2):89-96.

Arrow K, Solow R, Portney PR, Leamer EE, Radner R, and Schuman H (1993). Report of the NOAA Panel on Contingent Valuation.

AZOS working group (2006). Final report AZOS (In Dutch). Diemen: Health Care Insurance Board.

Banta HD (1982). Technology assessment and policy making. In Resources for health. Technology assessment for policy making, edited by HD Banta. New York: Praeger Publishers.

Barton GR, Bankart J and Davis AC (2005). A comparison of the quality of life of hearing-impaired people as estimated by three different utility measures. International Journal of Audiology 44(3):157-63.

Barton GR, Bankart J, Davis AC, and Summerfield QA (2004). Comparing Utility Scores Before and After Hearing-Aid Provision : Results According to the EQ-5D, HUI3 and SF-6D. Applied Health Economics and Health Policy 3(2):103-5.

Battista RN (1989). Innovation and diffusion of health-related technologies. A conceptual framework. International Journal of Technology Assessment in Health Care 5(2):227-48.

Battista RN and Hodge MJ (1999). The evolving paradigm of health technology assessment: reflections for the millennium. Canadian medical Association Journal 160(10):1464-7.

Bayoumi AM (2004). The measurement of contingent valuation for health economics. Pharmacoeconomics 22(11):691-700.

Bess FH (2000). The role of generic health-related quality of life measures in establishing audiological rehabilitation outcomes. Ear \& Hearing 21(4 Suppl):74S-79S.

Borisova NN and Goodman AC (2003). Measuring the value of time for methadone maintenance clients: willingness to pay, willingness to accept, and the wage rate. Health Economics 12(4):323-34.

Bosch JL and Hunink MG (2000). Comparison of the Health Utilities Index Mark 3 (HUI3) and the EuroQol EQ-5D in patients treated for intermittent claudication. Quality of Life Research 9(6):591-601.

Bosch M, van der Weijden T, Wensing M, and Grol R (2007). Tailoring quality improvement interventions to identified barriers: a multiple case analysis. Journal of Evaluation in Clinical Practice 13(2):161-8.

Brazier J and Deverill M (1999). A checklist for judging preference-based measures of health related quality of life: learning from psychometrics. Health Economics 8(1):41-51.

Brazier J, Roberts J and Deverill M (2002). The estimation of a preference-based measure of health from the SF-36. Journal of Health Economics 21(2):271-92.

Brazier J, Roberts J, Tsuchiya A, and Busschbach J (2004). A comparison of the EQ-5D and SF-6D across seven patient groups. Health Economics 13(9):873-84. 
Bridges JF and Jones C (2007). Patient-based health technology assessment: a vision of the future. International Journal of Technology Assessment in Health Care 23(1):30-5.

Briggs A, Claxton K and Sculpher M (2006). Decision modelling for health economic evaluation. Oxford: Oxford University Press.

Briggs AH and O'Brien BJ (2001). The death of cost-minimization analysis? Health Economics 10(2):179-84.

Briggs AH, Wonderling DE and Mooney CZ (1997). Pulling cost-effectiveness analysis up by its bootstraps: a non-parametric approach to confidence interval estimation. Health Economics 6(4):327-40.

British Academy of Audiology (2006). NHS Audiology - Building the Service. British Academy of Audiology.

Brooks R (1996). EuroQol: the current state of play. Health Policy 37(1):53-72.

Brown TC and Gregory R (1999). Why the WTA-WTP Disparity Matters. Ecological Economics 28(3):323-35.

Burstrom K, Johannesson M and Diderichsen F (2001). Swedish population health-related quality of life results using the EQ-5D. Quality of Life Research 10(7):621-35.

Buxton MJ (2006). Economic evaluation and decision making in the UK. Pharmacoeconomics 24(11):1133-42.

Cabana MD, Rand CS, Powe NR, Wu AW, Wilson MH, Abboud PA, and Rubin HR (1999). Why don't physicians follow clinical practice guidelines? A framework for improvement. Journal of the American Medical Association 282(15):1458-65.

Campbell H, Hotchkiss R, Bradshaw N, and Porteous M (1998). Integrated care pathways. British Medical Journal 316(7125):133-7.

Carthy T, Chilton S, Covey J, Hopkins L, Jones-Lee M, Loomes G, Pidgeon N, and Spencer A (1999). On the Contingent Valuation of Safety and the Safety of Contingent Valuation: Part 2--The CV/SG 'Chained' Approach. Journal of Risk and Uncertainty 17(3):187-213.

CBO (1998). Treatment and prevention of coronary heart disease through lowering serum cholesterol levels. Utrecht: National Organisation for Qality Assurance in Hospitals (CBO).

Central Bureau for Statistics (2007). StatLine database. Available at: http://www.cbs.nl/nl$\mathrm{NL} / \mathrm{menu} / \mathrm{cijfers} /$ statline/toegang/default.htm.

Chorus AMJ, Kremer A, Oortwijn WJ, and Schaapveld K (1995). Hearing impairment in the Netherlands (in Dutch). Leiden: TNO Preventie en Gezondheid.

Claxton K and Posnett J (1996). An economic approach to clinical trial design and research prioritysetting. Health Economics 5(6):513-24.

Claxton K, Sculpher M and Drummond M (2002). A rational framework for decision making by the National Institute For Clinical Excellence (NICE). Lancet 360(9334):711-5.

Cohen J (1988). Statistical power analysis for the behavioral sciences. Hillsdale, N.J.: Lawrence Erlbaum Associates.

College voor Zorgverzekeringen (2006). Guidelines for pharmaco-economic research (In Dutch). Diemen: College voor Zorgverzekeringen.

Conner-Spady B and Suarez-Almazor ME (2003). Variation in the estimation of quality-adjusted lifeyears by different preference-based instruments. Medical Care 41(7):791-801. 
Cookson R (2003). Willingness to pay methods in health care: a sceptical view. Health Economics 12:891-4.

Council for Public Health and Health Care (2006). Sensible and sustainable care (in Dutch). Zoetermeer: Council for Public Health and Health Care.

Cummings CW (2005). Otolaryngology: head \& neck surgery. Philadelphia, PA: Elsevier Mosby.

Dalton DS, Cruickshanks KJ, Klein BE, Klein R, Wiley TL, and Nondahl DM (2003). The impact of hearing loss on quality of life in older adults. Gerontologist 43(5):661-8.

Davis AC (1995). Hearing in Adults. London: Whurr Publishers Ltd.

Devlin N, Hansen P and Herbison P (2000). Variations in self-reported health status: results from a New Zealand survey. New Zealand Medical Journal 113(1123):517-20.

Diener A, O'Brien B and Gafni A (1998). Health care contingent valuation studies: a review and classification of the literature. Health Economics 7(4):313-26.

Dolan P (1997). Modeling valuations for EuroQol health states. Medical Care 35(11):1095-108.

Donaldson C, Thomas R and Torgerson DJ (1997). Validity of Open-Ended and Payment Scale Approaches to Eliciting Willingness to Pay. Applied Economics 29(1):79-84.

Dowie J (2004). Why cost-effectiveness should trump (clinical) effectiveness: the ethical economics of the South West quadrant. Health Economics 13(5):453-9.

Dowie J (2005). No room for kinkiness in a public healthcare system. Pharmacoeconomics 23(12):1203-5.

Drummond MF, Sculpher MJ, Torrance GW, O'Brien BJ, and Stoddard GL (2005). Methods for the Economic Evaluation of Health Care Programmes. New York: Oxford University Press.

Duijvestijn JA, Anteunis LJ, Hendriks JJ, and Manni JJ (1999). Definition of hearing impairment and its effect on prevalence figures. A survey among senior citizens. Acta Oto-Laryngologica 119(4):420-3.

Espallargues M, Czoski-Murray CJ, Bansback NJ, Carlton J, Lewis GM, Hughes LA, Brand CS, and Brazier JE (2005). The impact of age-related macular degeneration on health status utility values. Investigative Ophthalmology and Visual Science 46(11):4016-23.

Espeland A and Baerheim A (2003). Factors affecting general practitioners' decisions about plain radiography for back pain: implications for classification of guideline barriers--a qualitative study. BMC Health Services Research 3(1):8.

Feeny D, Furlong W, Boyle M, and Torrance GW (1995). Multi-attribute health status classification systems. Health Utilities Index. Pharmacoeconomics 7(6):490-502.

Feeny D, Furlong W, Torrance GW, Goldsmith CH, Zhu Z, DePauw S, Denton M, and Boyle M (2002). Multiattribute and single-attribute utility functions for the health utilities index mark 3 system. Medical Care 40(2):113-28.

Feeny D, Wu L and Eng K (2004). Comparing short form 6D, standard gamble, and Health Utilities Index Mark 2 and Mark 3 utility scores: results from total hip arthroplasty patients. Quality of Life Research 13(10):1659-70.

Felli JC and Hazen GB (1998). Sensitivity analysis and the expected value of perfect information. Medical Decision Making 18(1):95-109.

Fenwick E, Claxton K and Sculpher M (2001). Representing uncertainty: the role of cost-effectiveness acceptability curves. Health Economics 10(8):779-87. 
Fenwick E, Claxton K and Sculpher M (2007). The value of implementation and the value of information: combined and uneven development. Medical Decision Making In press.

Fenwick E, Palmer S, Claxton K, Sculpher M, Abrams K, and Sutton A (2006). An iterative Bayesian approach to health technology assessment: application to a policy of preoperative optimization for patients undergoing major elective surgery. Medical Decision Making 26(5):480-96.

Foy R, Walker A, Ramsay C, Penney G, Grimshaw J, and Francis J (2005). Theory-based identification of barriers to quality improvement: induced abortion care. International Journal for Quality in Health Care 17(2):147-55.

Frew EJ, Whynes DK and Wolstenholme JL (2003). Eliciting willingness to pay: comparing closedended with open-ended and payment scale formats. Medical Decision Making 23(2):150-9.

Gagnebin J and Maire R (2002). Infection screening in sudden and progressive idiopathic sensorineural hearing loss: a retrospective study of 182 cases. Otology \& Neurotology 23(2):160-2.

Grol R (1997). Personal paper. Beliefs and evidence in changing clinical practice. British Medical Journal 315(7105):418-21.

Grol R and Grimshaw J (2003). From best evidence to best practice: effective implementation of change in patients' care. Lancet 362(9391):1225-30.

Grol R and Wensing M (2004). What drives change? Barriers to and incentives for achieving evidencebased practice. Medical Journal of Australia 180(6 Suppl):S57-60.

Grol RP, Bosch MC, Hulscher ME, Eccles MP, and Wensing M (2007). Planning and studying improvement in patient care: the use of theoretical perspectives. Milbank Quarterly 85(1):93-138.

Grone O and Garcia-Barbero M (2001). Integrated care: A position paper of the WHO European office for integrated health care services. International Journal of Integrated Care 1:e21.

Groot Koerkamp B, Hunink MG, Stijnen T, and Weinstein MC (2006). Identifying key parameters in cost-effectiveness analysis using value of information: a comparison of methods. Health Economics 15(4):383-92.

Groot WNJ and Maassen van den Brink H (2006). Costs per life-year gained (in Dutch). In Insight in sensible and sustainable care, edited by Council for Public Health and Health Care. The Hague: Council for Public Health and Health Care.

Gross Portney L and Watkins MP (1993). Foundations of Clinical Research. Norwalk: Appleton \& Lange.

Grutters JPC, Joore MA, Van der Horst F, Verschuure J, Dreschler WA, and Anteunis LJC (2007a). Choosing between measures: comparison of EQ-5D, HUI2 and HUI3 in persons with hearing complaints. Quality of Life Research 16(8):1439-49.

Grutters JPC, Van der Horst F, Joore MA, Verschuure H, Dreschler WA, and Anteunis LJC (2007b). Potential barriers and facilitators for implementation of an integrated care pathway for hearingimpaired persons: an exploratory survey among patients and professionals. BMC Health Services Research 7(57).

Guyatt G, Jaeschke R, Feeny D, and Patrick D (1996). Measurements in Clinical Trials: Choosing the Right Approach. In Quality of Life and Pharmacoeconomics in Clinical Trials, edited by B Spilker, pp. 41-48. Philadelphia: Lippincott Williams \& Wilkins.

Haagen EC, Nelen WL, Hermens RP, Braat DD, Grol RP, and Kremer JA (2005). Barriers to physician adherence to a subfertility guideline. Human Reproduction 20(12):3301-6. 
Habbema JDF (1989). What is medical technology assessment? In Medical Technology Assessment and health policy (in Dutch), edited by JDF Habbema, AF Casparie, JH Mulder, and FFH Rutten. Alphen aan den Rijn: Samson Stafleu.

Hadley J (2000). Better health care decisions: fulfilling the promise of health services research. Health Services Research 35(1 Pt 2):175-86.

Hanemann WM (1991). Willingness to Pay and Willingness to Accept: How Much Can They Differ? American Economic Review 81 3:635-47.

Hanemann WM and Kanninen B (1998). The Statistical Analysis Of Discrete-Response CV Data. Berkeley: Department of Agricultural and Resource Economics and Policy

Hanley N, Ryan M and Wright R (2003). Estimating the Monetary Value of Health Care: Lessons from Environmental Economics. Health Economics 12 1:3-16.

Hansen J, Van der Velden LJF and Hingstman L (2005). Labour market monitor ENT-specialists 20052015/2020 (In Dutch). Utrecht: Nivel.

Hawthorne MR, Nunez DA, Clarke GP, and Robertshaw D (1991). Direct referral hearing aid provision in the over sixties age group. Journal of Laryngology \& Otology 105(10):825-7.

Health Care Insurance Board (2006). Guidelines for pharmaco-economic research (In Dutch). Diemen: Health Care Insurance Board.

Health Committee (2007a). Health - Written evidence. Available at: http://www.publications. parliament.uk/pa/cm200607/cmselect/cmhealth/392/392we01.htm.

Health Committee (2007b). New Inquiry and terms of reference: Audiology. Available at: http://www.parliament.uk/parliamentary_committees/health_committee/hcpn070112.cfm.

Hicks JR (1943). The Four Conumer's Surpluses. Review of Economic Studies 11(1):31-41.

Hofhuis A, van der Giessen JW, Borgsteede FH, Wielinga PR, Notermans DW, and van Pelt W (2006). Lyme borreliosis in the Netherlands: strong increase in GP consultations and hospital admissions in past 10 years. EuroSurveillance 11(6):E060622 2.

Holland R, Smith RD, Harvey I, Swift L, and Lenaghan E (2004). Assessing quality of life in the elderly: a direct comparison of the EQ-5D and AQoL. Health Economics 13(8):793-805.

Hoomans T, Ament A, Evers S, and Severens JL (2007). Worthwhile Implementation of Evidence-Based Guidelines into Clinical Practice: How to Determine the Investment Potential for Guideline Implementation and the Value for Money of Implementation Strategies? Presented at 6th World Congress of the International Health Economics Association (iHEA). Copenhagen, Denmark.

Horowitz JK and McConnell KE (2002). A Review of WTA/WTP Studies. Journal of Environmental Economics and Management 44 3:426-47.

Huber J and Zwerina K (1996). The Importance of Utility Balance in Efficient Choice Designs. Journal of Marketing Research 33:307-17.

Johansson MS and Arlinger SD (2003). Prevalence of hearing impairment in a population in Sweden. International Journal of Audiology 42(1):18-28.

Joore MA, Brunenberg DE, Chenault MN, and Anteunis LJ (2003a). Societal effects of hearing aid fitting among the moderately hearing impaired. International Journal of Audiology 42(3):152-60.

Joore MA, Van Der Stel H, Peters HJ, Boas GM, and Anteunis LJ (2003b). The cost-effectiveness of hearing-aid fitting in the Netherlands. Archives of Otolaryngology -- Head \& Neck Surgery 129(3):297-304. 
Kahneman D and Tversky A (1979). Prospect Theory: An Analysis of Decision under Risk. Econometrica 47 2:263-91.

Kaplan RM, Groessl EJ, Sengupta N, Sieber WJ, and Ganiats TG (2005). Comparison of measured utility scores and imputed scores from the SF-36 in patients with rheumatoid arthritis. Medical Care 43(1):79-87.

Kind P, Dolan P, Gudex C, and Williams A (1998). Variations in population health status: results from a United Kingdom national questionnaire survey. British Medical Journal 316(7133):736-41.

Kjaer T, Bech M, Gyrd-Hansen D, and Hart-Hansen K (2006). Ordering effect and price sensitivity in discrete choice experiments: need we worry? Health Economics 15(11):1217-28.

Klose T (1999). The contingent valuation method in health care. Health Policy 47(2):97-123.

Koay CB and Sutton GJ (1996). Direct hearing aid referrals: a prospective study. Clinical Otolaryngology \& Allied Sciences 21(2):142-6.

Kodner DL and Spreeuwenberg C (2002). Integrated care: meaning, logic, applications, and implications--a discussion paper. International Journal of Integrated Care 2:e12.

Kopec JA and Willison KD (2003). A comparative review of four preference-weighted measures of health-related quality of life. Journal of Clinical Epidemiology 56(4):317-25.

Lamers LM, Stalmeier PF, McDonnell J, Krabbe PF, and van Busschbach JJ (2005). Kwaliteit van leven meten in economische evaluaties: het Nederlands EQ-5D-tarief. Nederlands Tijdschrift voor Geneeskunde 149(28):1574-8.

Lancsar E and Louviere J (2006). Deleting 'irrational' responses from discrete choice experiments: a case of investigating or imposing preferences? Health Economics 15(8):797-811.

Larson VD, Williams DW, Henderson WG, Luethke LE, Beck LB, Noffsinger D, Wilson RH, Dobie RA, Haskell GB, Bratt GW, Shanks JE, Stelmachowicz P, Studebaker GA, Boysen AE, Donahue A, Canalis R, Fausti SA, and Rappaport BZ (2000). Efficacy of 3 commonly used hearing aid circuits: A crossover trial. NIDCD/VA Hearing Aid Clinical Trial Group. Journal of the American Medical Association 284(14):1806-13.

Longworth L and Bryan S (2003). An empirical comparison of EQ-5D and SF-6D in liver transplant patients. Health Economics 12(12):1061-7.

Louviere JJ, Hensher DA and Swait JD (2000). Stated choice methods: analysis and applications. Cambridge: Cambridge University Press.

Lubetkin El, Jia H, Franks P, and Gold MR (2005). Relationship among sociodemographic factors, clinical conditions, and health-related quality of life: examining the EQ-5D in the U.S. general population. Quality of Life Research 14(10):2187-96.

Marra CA, Esdaile JM, Guh D, Kopec JA, Brazier JE, Koehler BE, Chalmers A, and Anis AH (2004). A comparison of four indirect methods of assessing utility values in rheumatoid arthritis. Medical Care 42(11):1125-31.

Marra CA, Woolcott JC, Kopec JA, Shojania K, Offer R, Brazier JE, Esdaile JM, and Anis AH (2005). A comparison of generic, indirect utility measures (the HUI2, HUI3, SF-6D, and the EQ-5D) and disease-specific instruments (the RAQoL and the HAQ) in rheumatoid arthritis. Social Science \& Medicine 60(7):1571-82.

Martens P (2002). Health transitions in a globalising world: towards more disease or sustained health? Futures 34:635-48. 
Maxwell RJ (1981). Health and wealth: an international study of health-care spending. Lexington: Lexington Books.

McIntosh E (2006). Using discrete choice experiments within a cost-benefit analysis framework: some considerations. Pharmacoeconomics 24(9):855-68.

Meister H, Lausberg I, Walger M, and von Wedel H (2001). Using conjoint analysis to examine the importance of hearing aid attributes. Ear \& Hearing 22(2):142-50.

Mulrow CD, Aguilar C, Endicott JE, Tuley MR, Velez R, Charlip WS, Rhodes MC, Hill JA, and DeNino LA (1990). Quality-of-life changes and hearing impairment. A randomized trial. Annals of Internal Medicine 113(3):188-94.

Mur-Veeman I, Eijkelberg I and Spreeuwenberg C (2001). How to manage the implementation of shared care: a discussion of the role of power, culture and structure in the development of shared care arrangements. Journal of Management in Medicine 15(2):142-55.

Nancarrow SA and Borthwick AM (2005). Dynamic professional boundaries in the healthcare workforce. Sociology of Health \& IIIness 27(7):897-919.

National Institute for Clinical Excellence (2004). Guide to the Methods of Technology Appraisal. London: National Institute for Clinical Excellence.

Nationale Raad voor de Volksgezondheid \& College voor Ziekenhuisvoorzieningen (1995). Transmural somatic care (in Dutch). Zoetermeer: Nationale Raad voor de Volksgezondheid.

Norris P (2001). How 'we' are different from 'them': occupational boundary maintenance in the treatment of musculo-skeletal problems. Sociology of Health \& Illness 23(1):24-43.

O'Brien B and Gafni A (1996). When do the "dollars" make sense? Toward a conceptual framework for contingent valuation studies in health care. Medical Decision Making 16(3):288-99.

O'Brien BJ, Gertsen K, Willan AR, and Faulkner LA (2002). Is there a kink in consumers' threshold value for cost-effectiveness in health care? Health Economics 11(2):175-80.

O'Brien BJ, Goeree R, Gafni A, Torrance GW, Pauly MV, Erder H, Rusthoven J, Weeks J, Cahill M, and LaMont B (1998). Assessing the value of a new pharmaceutical. A feasibility study of contingent valuation in managed care. Medical Care 36(3):370-84.

O'Brien BJ, Spath M, Blackhouse G, Severens JL, Dorian P, and Brazier J (2003). A view from the bridge: agreement between the SF-6D utility algorithm and the Health Utilities Index. Health Economics 12(11):975-81.

Olsen JA and Donaldson C (1998). Helicopters, hearts and hips: using willingness to pay to set priorities for public sector health care programmes. Social Science \& Medicine 46(1):1-12.

Onwujekwe O (2004). Criterion and content validity of a novel structured haggling contingent valuation question format versus the bidding game and binary with follow-up format. Social Science \& Medicine 58:525-37.

Onwujekwe O, Hanson K and Fox-Rushby J (2005). Do divergences between stated and actual willingness to pay signify the existence of bias in contingent valuation surveys? Social Science \& Medicine 60:525-36.

Oostenbrink JB, Koopmanschap MA and Rutten FFH (2004). Manual for costing research (in Dutch). Amstelveen: College voor zorgverzekeringen.

Oostenbrink R, Moll HA and Essink-Bot ML (2002). The EQ-5D and the Health Utilities Index for permanent sequelae after meningitis: a head-to-head comparison. Journal of Clinical Epidemiology 55(8):791-9. 
Parmet S, Lynm C and Glass RM (2007). JAMA patient page. Adult hearing loss. Journal of the American Medical Association 298(1):130.

Petrou S and Hockley C (2005). An investigation into the empirical validity of the EQ-5D and SF-6D based on hypothetical preferences in a general population. Health Economics 14(11):1169-89.

Phillips KA, Johnson FR and Maddala T (2002). Measuring what people value: a comparison of "attitude" and "preference" surveys. Health Services Research 37(6):1659-79.

Pickard AS, Johnson JA and Feeny DH (2005). Responsiveness of generic health-related quality of life measures in stroke. Quality of Life Research 14(1):207-19.

Platform HTA (2001). Promotion of HTA in the Netherlands (in Dutch). The Hague: Raad voor Gezondheidsonderzoek.

Popelka MM, Cruickshanks KJ, Wiley TL, Tweed TS, Klein BE, and Klein R (1998). Low prevalence of hearing aid use among older adults with hearing loss: the Epidemiology of Hearing Loss Study. Journal of the American Geriatrics Society 46(9):1075-8.

Porter M and Macintyre S (1984). What is, must be best: a research note on conservative or deferential responses to antenatal care provision. Social Science \& Medicine 19(11):1197-200.

Propp JM, McCarthy BJ, Davis FG, and Preston-Martin S (2006). Descriptive epidemiology of vestibular schwannomas. Neuro-Oncology 8(1):1-11.

Rasanen P, Roine E, Sintonen H, Semberg-Konttinen V, Ryynanen OP, and Roine R (2006). Use of quality-adjusted life years for the estimation of effectiveness of health care: A systematic literature review. International Journal of Technology Assessment in Health Care 22(2):235-41.

Ratcliffe J (2000). The use of conjoint analysis to elicit willingness-to-pay values. Proceed with caution? International Journal of Technology Assessment in Health Care 16(1):270-5.

Ratcliffe J and Buxton M (1999). Patients' preferences regarding the process and outcomes of lifesaving technology. An application of conjoint analysis to liver transplantation. International Journal of Technology Assessment in Health Care 15(2):340-51.

Ratcliffe J and Longworth L (2002). Investigating the structural reliability of a discrete choice experiment within health technology assessment. International Journal of Technology Assessment in Health Care 18(1):139-44.

Rip A, Schot JW and Misa TJ (1995). Constructive Technology Assessment: A New Paradigm for Managing Technology in Society. In Managing technology in society: the approach of constructive technology assessment, edited by A Rip, TJ Misa, and JW Schot. London: Pinter.

Robertson N, Baker R and Hearnshaw H (1996). Changing the clinical behavior of doctors: a psychological framework. Quality in Health Care 5(1):51-4.

Ryan M (2004). Discrete choice experiments in health care. British Medical Journal 328(7436):360-1.

Ryan M and Farrar S (2000). Using conjoint analysis to elicit preferences for health care. British Medical Journal 320(7248):1530-3.

Ryan M and Gerard K (2003). Using discrete choice experiments to value health care programmes: current practice and future research reflections. Applied Health Economics \& Health Policy 2(1):55-64 .

Ryan M and Hughes J (1997). Using conjoint analysis to assess women's preferences for miscarriage management. Health Economics 6(3):261-73.

Ryan M, Major K and Skatun D (2005). Using discrete choice experiments to go beyond clinical outcomes when evaluating clinical practice. Journal of Evaluation in Clinical Practice 11(4):328-38. 
Ryan M, McIntosh E and Shackley P (1998). Methodological issues in the application of conjoint analysis in health care. Health Economics 7(4):373-8.

Ryan M, Ratcliffe J and Tucker J (1997). Using willingness to pay to value alternative models of antenatal care. Social Science \& Medicine 44(3):371-80.

Ryan M, Scott DA and Donaldson C (2004). Valuing health care using willingness to pay: a comparison of the payment card and dichotomous choice methods. Health Economics 23:237-58.

Ryan M, Scott DA, Reeves C, Bate A, van Teijlingen ER, Russell EM, Napper M, and Robb CM (2001). Eliciting public preferences for healthcare: a systematic review of techniques. Health Technology Assessment 5(5):1-186.

Ryan M and Ubach C (2003). Testing for an Experience Endowment Effect in Health Care. Applied Economics Letters 10 7:407-10.

Salkeld G, Ryan M and Short L (2000). The veil of experience: do consumers prefer what they know best? Health Economics 9(3):267-70.

Salomon JA, Mathers CD, Chatterji S, Sadana R, Bedirhan Üstün T, and Murray CJL (2003). Quantifying individual levels of health: definitions, concepts, and measurement issues. In Health systems performance assessment: debates, methods and empiricism., edited by CJL Murray and DB Evans. Geneva: World Health Organization.

Severens JL, Brunenberg DE, Fenwick EA, O'Brien B, and Joore MA (2005). Cost-effectiveness acceptability curves and a reluctance to lose. Pharmacoeconomics 23(12):1207-14.

Slothuus Skjoldborg U and Gyrd-Hansen D (2003). Conjoint analysis. The cost variable: an Achilles' heel? Health Economics 12(6):479-91.

Smith RD (2000). The discrete-choice willingness-to-pay question format in health economics: should we adopt environmental guidelines? Medical Decision Making 20(2):194-206.

Spreeuwenberg C and Pop P (2000). Transmural care (in Dutch). In Handbook transmural care, edited by C Spreeuwenberg, P Pop, GHMI Beusmans, RAG Winkens, and H Van Zutphen. Maarssen: Elsevier Healthcare.

Stephens D (1987). Audiological rehabilitation. In Scott Brown's Otolaryngology. Vol. 2: Adult Audiology, edited by D Stephens.

Stephens D, Lewis P, Davis A, Gianopoulos I, and Vetter N (2001). Hearing aid possession in the population: lessons from a small country. Audiology 40(2):104-11.

Stevens F, Van der Horst F, Grit F, and Diederiks J (2007). Exclusive, idiosyncratic and collective expertise in the inter-professional arena. The case of optometry and eye care in the Netherlands. Sociology of Health \& Illness 29(4):481-96.

Street DJ, Burgess L and Louviere JJ (2005). Quick and easy choice sets: Constructing optimal and nearly optimal stated choice experiments. International Journal of Research in Marketing 22(4):459-70.

Streiner DL and Norman GR (1995). Health measurements scales: a practical guide to their development and use. Oxford: Oxford University Press.

Swan IR and Browning GG (1994). A prospective evaluation of direct referral to audiology departments for hearing aids. Journal of Laryngology \& Otology 108(2):120-4.

Telser $\mathrm{H}$ and Zweifel P (2006). A new role for consumers' preferences in the provision of health care. Economic affairs 26(3):4-9. 
The British Society of Hearing Aid Audiologists (2005). Audiological provision in Europe. A publicprivate partnership? London: BSHAA Council.

The EuroQol Group (1990). EuroQol--a new facility for the measurement of health-related quality of life. Health Policy 16(3):199-208.

Torrance GW, Feeny DH, Furlong WJ, Barr RD, Zhang Y, and Wang Q (1996). Multiattribute utility function for a comprehensive health status classification system. Health Utilities Index Mark 2. Medical Care 34(7):702-22.

Train KE (2003). Discrete choice methods with simulation. Cambridge: University Press.

Tugwell P, Bennett K, Feeny D, Guyatt G, and Haynes RB (1986). A framework for the evaluation of technology: the technology assessment iterative loop. In Health care technology: effectiveness, efficiency \& public policy, edited by D Feeny, G Guyatt, and P Tugwell. Montreal: Institute for Research on Public Policy.

Ubach C, Scott A, French F, Awramenko M, and Needham G (2003). What do hospital consultants value about their jobs? A discrete choice experiment. British Medical Journal 326(7404):1432.

Valente M (2006). Guideline for Audiologic Management of the Adult Patient. Available at: http://www.audiologyonline.com/articles/pf_article_detail.asp?article_id=1716.

Van Bokhoven T, Kok G and Van der Weijden T (2004). Designing a quality improvement intervention: a systematic approach. In Quality improvement research. Understanding the science of change in health care., edited by R Grol, R Baker, and F Moss. London: BMJ Books.

Van den Berg B, Al M, Brouwer W, Van Exel J, and Koopmanschap M (2005a). Economic valuation of informal care: the conjoint measurement method applied to informal caregiving. Social Science \& Medicine 61(6):1342-55.

Van den Berg B, Bleichrodt $\mathrm{H}$ and Eeckhoudt L (2005b). The economic value of informal care: a study of informal caregivers' and patients' willingness to pay and willingness to accept for informal care. Health Economics 14(4):363-76.

Van der Pol M and Cairns J (1998). Establishing patient preferences for blood transfusion support: an application of conjoint analysis. Journal of Health Services Research \& Policy 3(2):70-6.

Van der Velden LJF and Hingstman L (2000). Demand estimate ENT-specialists 1999-2010 (In Dutch). Utrecht: Nivel.

Van Hout BA, Al MJ, Gordon GS, and Rutten FF (1994). Costs, effects and C/E-ratios alongside a clinical trial. Health Economics 3(5):309-19.

Van Wijmen FCB, Spreeuwenberg C and Willemse DCM (2000). Substitution (in Dutch). In Handbook transmural care, edited by C Spreeuwenberg, P Pop, GHMI Beusmans, RAG Winkens, and H Van Zutphen. Maarssen: Elsevier Healthcare.

Veldhuizen JA, Theunissen EJJM and Dreschler WA (2002). The NOAH protocol hearing aid provision (In Dutch, available upon request from the author). Leiden: Nationaal Overleg Audiologische Hulpmiddelen.

Vuorialho A, Karinen P and Sorri M (2006a). Counselling of hearing aid users is highly cost-effective. European Archives of Otorhinolaryngology 263(11):988-95.

Vuorialho A, Karinen P and Sorri M (2006b). Effect of hearing aids on hearing disability and quality of life in the elderly. International Journal of Audiology 45(7):400-5.

Wagner EH, Austin BT and Von Korff M (1996). Organizing care for patients with chronic illness. Milbank Quarterly 74(4):511-44. 
Walton SM, Graves PE, Mueser PR, and Dow JK (2002). The bias against new innovations in health care: value uncertainty and willingness to pay. Value in Health 5(2):67-70.

Weinstein MC (2006). Recent developments in decision-analytic modelling for economic evaluation. Pharmacoeconomics 24(11):1043-53.

Weinstein MC, O'Brien B, Hornberger J, Jackson J, Johannesson M, McCabe C, and Luce BR (2003). Principles of good practice for decision analytic modeling in health-care evaluation: report of the ISPOR Task Force on Good Research Practices--Modeling Studies. Value in Health 6(1):9-17.

Wensing M and Elwyn G (2004). Research on patients' views in the evaluation and improvement of quality of care. In Quality improvement research. Understanding the science of change in health care, edited by R Grol, R Baker, and F Moss. London: BMJ Books.

Wensing M, Laurant M, Hulscher M, and Grol R (1999). Methods for identifying barriers and facilitators for implementation. In Changing professional practice. Theory and practice of clinical guidelines implementation, edited by T Thorsen and M Mäkelä. Copenhagen: DSI.

Whynes DK, Frew E and Wolstenholme JL (2003). A comparison of two methods for eliciting contingent valuations of colorectal cancer screening. Journal of Health Economics 22(4):555-74.

Whynes DK, Wolstenholme JL and Frew E (2004). Evidence of range bias in contingent valuation payment scales. Health Economics 13(2):183-90.

Winkens RAG and Klazinga NS (2000). Scientific research (in Dutch). In Handbook Transmural Care, edited by C Spreeuwenberg, P Pop, GHMI Beusmans, RAG Winkens, and H Van Zutphen. Maarssen: Elsevier Healthcare.

World Health Organization (1946). Constitution of the World Health Organization. Geneva: World Health Organization.

Ziekenfondsraad (1994). Provision of hearing aids (in Dutch). Amstelveen: Ziekenfondsraad. 

Summary 

The increasing burden of chronic diseases on today's health care system has resulted in an increased importance of redesigning health care to patient-centered care, and of attuning the tasks of health care professionals. With regard to hearing aid provision, the current growth of the ageing population causes a proportional growth of the population with hearing impairment. This raises the burden of disease and the medical consumption for hearing impairment and, as a consequence, increases the costs of hearing rehabilitation. Increasing attention is therefore paid to attuning the tasks of all professionals involved in the care for hearing-impaired persons. This has resulted in shared care initiatives in hearing aid provision, where some of the tasks of Ear, Nose and Throat (ENT) specialists and Audiological Centres are substituted to hearing aid dispensers. This would allow for direct hearing aid provision by dispensers, without prescription or approval of an ENT-specialist or Audiological Centre.

Health technology assessment seeks to inform health policy makers by examining medical, social, ethical and economic implications of technologies in health care. Health technology assessment is an iterative process, and the assessment needs to continue throughout the life of a technology. This iterative process is illustrated by the Technology Assessment Iterative Loop. The aim of the thesis is to use health technology assessment to evaluate whether an organizational innovation, shared care in hearing aid provision, benefits the care for hearing-impaired persons.

\section{Part I}

The first part of this thesis focuses on barriers and facilitators with regard to the implementation of shared care in hearing aid provision in an early stage of an organizational innovation process, to become aware of which issues are of importance in the field, and to guide the design process of the innovation.

In Chapter 2 we examined expectations and potential barriers and facilitators of hearing impaired persons and professionals involved in the care for hearing impaired persons, using a survey. On average general practitioners and hearing aid dispensers had positive expectations towards shared care in hearing aid provision, while ENT-specialists and clinical audiologists had negative expectations. Most professionals either supported implementation of shared care, provided that a number of conditions were satisfied, or did not support implementation, unless roughly the same conditions were satisfied. Persons with hearing complaints evaluated the current organization of hearing aid provision and shared care equally, and stated to have confidence in the trained hearing aid dispenser. Many, especially older, participants stated however that they would still visit the general practitioner and ENT-specialist, even when this would not be necessary for reimbursement of the hearing aid, and found it important that the ENT-specialist or Audiological Centre evaluated their hearing aid. Overall, Chapter 2 identified professional concerns about shared care in hearing aid provision. Gaps in expectations exist amongst professions, but also amongst hearing impaired persons, especially between age groups and regions. Implementation on a regional level is recommended to best satisfy the stated conditions. 


\section{Part II}

In the second part of this thesis different outcome measures for shared care in hearing aid provision are considered, both in terms of health state utilities and monetary valuations.

In Chapter 3 utility scores were elicited in a population with hearing complaints, before and after hearing aid provision. Utility scores were elicited using the preference-based utility measures EuroQol 5D (EQ-5D), Health Utilities Index mark II (HUI2) and Health Utilities Index mark III (HUI3). These instruments differ in their underlying assumptions about what constitutes health state utility. Practicality, construct validity, agreement, responsiveness and impact on the incremental cost-effectiveness ratio for hearing aid fitting of the different measures were examined. Regarding practicality, all measures were found to have high completion rates. Both the EQ-5D and the HUI3 showed some evidence of construct validity. The HUI2 and HUI3 scores were found to be lower than the EQ-5D scores and agreement was moderate to poor. As to responsiveness, only the HUI2 and the HUI3 measured statistically significant improvement after hearing aid fitting, while the EQ$5 \mathrm{D}$ did not capture this effect. The incremental cost-effectiveness ratios for hearing aid fitting varied from € 647209 per Quality Adjusted Life Year (QALY) using the EQ-5D to $€$ 15811 per QALY using the HUI3. The results of this study show that utility scores, utility gain and incremental cost-effectiveness ratios heavily depend on the measure that is used to elicit health state utility. Chapter 3 indicates HUI3 as the instrument of first choice when measuring utility in a population with hearing complaints, but emphasizes the importance of a clear notion of what constitutes health state utility with regard to economic analyses.

Chapter 4 examined contingent valuation to elicit monetary values for hearing aid fitting. It provides additional evidence on the validity of two willingness to pay (WTP) elicitation formats: the open-ended question and the payment scale. We elicited WTP for a hearing aid among hearing aid users, using both a payment scale and an open-ended question. We compared the results from both formats, and tested criterion validity by comparing both formats with the actual out-of-pocket payment. Construct validity was tested by examining whether WTP was consistent with positive income elasticity. We found no statistically significant difference between the results on the payment scale and open-ended question. Both formats showed good criterion validity, although the open-ended question showed a stronger association with the actual out-of-pocket payment. The open-ended format showed better construct validity, as it was positively influenced by family income. The results of Chapter 4 indicate that the open-ended question was more valid than the payment scale question. We therefore recommend that in future WTP studies on hearing aids the open-ended question is used to directly elicit WTP values. The same recommendation may apply to other studies where respondents are familiar with costs or payments for the intervention under evaluation.

Chapter 5 focused on the disparity between willingness to accept (WTA) and WTP in discrete choice experiments. Our main objective was to compare WTA and WTP in a discrete choice experiment on hearing aid provision. Additionally, income effect and endowment effect were explored as possible explanations for the disparity between WTA and WTP, and the impact of using a WTA and/or WTP format to elicit monetary valuations on the net benefit of shared care in hearing aid provision was examined. Persons with 
hearing complaints randomly received a WTP (cost attribute defined as extra payment) or WTA (cost attribute defined as discount) version of the experiment. In the versions, except for the cost attribute, all choice sets were equal. The cost coefficient was found to be statistically significantly higher in the WTP format. Marginal WTA was statistically significantly higher than marginal WTP for two out of four attributes. The disparity between WTA and WTP was higher in the high educational (as proxy for income) group. We did not find proof of an experience endowment effect. Implementing a form of shared care in hearing aid provision would only be recommended when using WTP. From Chapter 5 it is clear that WTA exceeds WTP, also in a discrete choice experiment. As this affects monetary valuations, more research on when to use a payment or a discount in the cost attribute is needed before discrete choice results can be used in cost-benefit analyses.

Chapter 6 elicited patient preferences for transferring elements of hearing aid provision from the medical sector (ENT-specialists and Audiological Centres) to hearing aid dispensers, to understand the trade-offs between different elements of hearing aid provision. Participants preferred the initial assessment at the hearing aid dispenser, higher accuracy in identifying persons in need of medical care, shorter duration of the total hearing aid provision, and a follow-up at the ENT-specialist. They required compensation of at least $€ 17$ per two months extra duration, $€ 54$ for an initial assessment at the ENTspecialist, $€ 119$ per $10 \%$ decrease in accuracy, and $€ 227$ to forgo the follow-up at the ENT-specialist. Preferences were influenced by sex, age, educational level, and experience with hearing aid provision. From Chapter 6 it was found that hearing-impaired persons are receptive to transferring elements of hearing aid provision from the medical sector to dispensers. In the organization of hearing aid provision hearing-impaired persons prefer an initial assessment at a dispenser when the dispenser is at least $95 \%$ as accurate as the ENTspecialist, and prefer a follow-up visit at the ENT-specialist.

\section{Part III}

The third part of this thesis integrates all available evidence in a decision-analytic model to make a trade-off between the benefits and risks of shared care in hearing aid provision.

Chapter 7 describes the results of modeling the long-term cost-effectiveness of (different forms of) shared care as opposed to the current organization of hearing aid provision, for persons aged 50 years and older. The current organization of hearing aid provision as compared to shared care is somewhat more effective and somewhat more costly, and had a higher probability of being cost-effective (54\%). Of the alternative forms of shared care, a pathway with an initial assessment at the hearing aid dispenser and a follow-up at the ENTspecialist or Audiological Centre had the highest probability of being cost-effective $(40 \%)$. It is worthwhile to perform additional research to reduce the uncertainty surrounding these results. Additional research is especially worthwhile on whether persons seek help for their hearing complaints sooner in a shared care pathway and whether the hearing aid dispenser is capable of detecting pathology. 
In Chapter 8 the main findings of this thesis are summarized and discussed. Additionally, some methodological aspects are considered. First, it is questioned whether the Technology Assessment Iterative Loop is readily applicable to organizational innovations such as shared care in hearing aid provision. That is, organizational innovations often need implementation before they can be evaluated. Moreover, decision-analytical modeling turned out to be a useful tool to examine efficiency in a very early stage, even before conclusive evidence on efficacy is available. Next, randomized trials are rarely applicable to organizational innovations. Therefore pragmatic trials, which have limitations as well as advantages, are often more appropriate. Also, it is suggested that aspects of constructive technology assessment could be used to adapt the traditional health technology assessment to organizational innovation. These aspects are for example the introduction of technology assessment at a very early stage in the development of a technology, and a continuous dialogue between users of the technology. Finally, the fact that modeling is always a simplification of a complex reality, as well as the fact that monetary valuation is able to encompass much more than traditional quality adjusted life years and might therefore be more appropriate in evaluating organizational innovation, are considered.

Furthermore a number of recommendations are made in Chapter 8. Besides the recommendations directly based on the results of the studies, it is recommended that hearing aid dispensers develop a code of conduct. Also, it is recommended that health technology assessment should occupy a more important place in the process of organizational innovation in health care. More specifically, it is recommended that in organizational innovations in health care, health technology assessment should be applied from a very early stage.

Finally, areas for future research are discussed. These include consensus with regard to the evaluative space in economic evaluations in health care: should quality adjusted life years remain the recommended outcome measure, or is a broader view indicated? Additionally, more evidence is needed on the validity of directly measuring WTP, as well as on why the disparity between WTA and WTP occurs in a discrete choice experiment and when to use a discount or a payment in the cost attribute. Also, important areas for future research are whether hearing aid dispensers are capable of identifying patients in need of medical care, and on whether shared care in hearing aid provision will lower barriers for help-seeking. 
Samenvatting 

Chronische ziekten oefenen een toenemende druk uit op het huidige gezondheidszorgsysteem. Dit heeft ertoe geleid dat steeds meer belang wordt gehecht aan het herontwerpen van de gezondheidszorg tot patiëntgerichte zorg, en aan het onderling afstemmen van taken van professionals in de gezondheidszorg. De vergrijzing van de bevolking leidt tot een groeiend aantal slechthorenden. Dit verhoogt de ziektelast en medische consumptie met betrekking tot slechthorendheid, met stijgende kosten van gehoorrevalidatie als gevolg. Er wordt daarom steeds meer aandacht besteed aan het onderling afstemmen van de taken van alle betrokken beroepsbeoefenaren in de zorg voor slechthorenden. Zo is er steeds meer aandacht voor ketenzorg voor oudere slechthorenden, waarbij taken van Keel-, Neusen Oorartsen en Audiologische Centra overgenomen worden door audiciens. Audiciens kunnen bij slechthorenden die geen medische zorg nodig hebben dan direct een hoortoestel aanpassen.

Health technology assessment is een manier om nieuwe technologieën in de gezondheidszorg (uiteenlopend van medicijnen tot organisatieveranderingen) te evalueren. Het onderzoekt de medische, sociale, ethische en economische implicaties van technologieën en is gericht op besluitvorming. Health technology assessment is een iteratief proces, dat gedurende het gehele leven van een technologie dient te worden voortgezet. Dit iteratieve proces wordt geïllustreerd door de Technology Assessment Iterative Loop. Het doel van dit proefschrift is om door middel van health technology assessment te evalueren of ketenzorg in de hoortoestelverstrekking bevorderlijk is voor de zorg voor slechthorenden.

\section{Deel I}

Het eerste deel van dit proefschrift richt zich op belemmerende en bevorderende factoren bij het implementeren van ketenzorg in de hoortoestelverstrekking. Door deze factoren in een vroeg stadium van een organisatievernieuwingsproces te verkennen kan inzicht verkregen worden in wat er speelt in het veld, en kan de ontwikkeling van de vernieuwing gestuurd worden.

In Hoofdstuk $\mathbf{2}$ onderzochten we verwachtingen en potentiële belemmerende en bevorderende factoren bij slechthorenden en beroepsbeoefenaren betrokken bij de zorg voor slechthorenden, door middel van een survey. Over het algemeen hadden huisartsen en audiciens positieve verwachtingen van ketenzorg in de hoortoestelverstrekking, terwijl KNO-artsen en audiologen negatieve verwachtingen hadden. De meeste beroepsbeoefenaren gaven aan wel achter implementatie van de ketenzorg te staan, mits aan bepaalde voorwaarden werd voldaan, of niet achter implementatie te staan tenzij aan ongeveer dezelfde voorwaarden werd voldaan. Slechthorenden beoordeelden de huidige zorg voor slechthorenden en ketenzorg als gelijkwaardig, en gaven aan vertrouwen te hebben in de bijgeschoolde audicien. Veel, met name oudere, respondenten gaven echter aan dat ze ook naar de huisarts en KNO-arts zouden gaan als dat niet nodig zou zijn voor vergoeding van het hoortoestel. Zij vonden het belangrijk dat hun hoortoestelaanpassing werd geëvalueerd door een KNO-arts of een Audiologisch Centrum. Uit Hoofdstuk 2 bleek 
over het algemeen dat er bij de verschillende beroepsgroepen bedenkingen bestaan over ketenzorg in de hoortoestelverstrekking. Verschillen in verwachtingen bestaan tussen de beroepsgroepen, maar ook tussen de slechthorenden zelf, met name tussen verschillende leeftijdsgroepen en regio's. Implementatie op regionaal niveau wordt aanbevolen om het beste aan de genoemde voorwaarden te kunnen voldoen.

\section{Deel II}

In het tweede deel van dit proefschrift worden verschillende uitkomstmaten voor ketenzorg in de hoortoestelverstrekking in beschouwing genomen, zowel in termen van utiliteiten als monetaire waarderingen.

In Hoofdstuk 3 zijn utiliteitsscores gemeten in een populatie met gehoorklachten, voor en na hoortoestelaanpassing. Utiliteitsscores werden gemeten met de utiliteitsinstrumenten EuroQol 5D (EQ-5D), Health Utilities Index mark II (HUI2) en Health Utilities Index mark III (HUI3). Deze instrumenten verschillen in hun veronderstellingen over hoe een utiliteit gevormd wordt. De bruikbaarheid, constructvaliditeit, overeenstemming, responsiviteit en de impact op de incrementele kosteneffectiviteitsratio van hoortoestelaanpassing van de verschillende meetinstrumenten werd onderzocht. Met betrekking tot de bruikbaarheid bleken de meetinstrumenten allemaal goed ingevuld te worden. Zowel de EQ-5D als de HUI3 vertoonden tekenen van constructvaliditeit. De HUI2 en HUI3 scores waren lager dan de EQ-5D scores, en de overeenstemming was redelijk tot slecht. Wat de responsiviteit betreft vonden alleen de HUI2 en HUI3 een statistisch significante verbetering na hoortoestelaanpassing, terwijl dit effect met de EQ-5D niet werd gevonden. De incrementele kosteneffectiviteitsratio's varieerden van € 647209 per voor kwaliteit gecorrigeerd levensjaar met de EQ-5D tot $€ 15811$ per voor kwaliteit gecorrigeerd levensjaar met de HUI3. De resultaten van deze studie tonen aan dat utiliteitsscores, utiliteitswinst en incrementele kosteneffectiviteitsratio's sterk afhankelijk zijn van het meetinstrument dat gebruikt is om de utiliteiten te meten. Hoofdstuk 3 wijst de HUI3 aan als instrument van voorkeur voor het meten van utiliteiten in een populatie met gehoorklachten, maar benadrukt het belang van een duidelijk begrip over wat, met betrekking tot economische analyses, een utiliteit vormt.

Hoofdstuk 4 onderzocht de contingent valuation methode om monetaire waarderingen te meten. Het hoofdstuk verschaft aanvullend bewijs met betrekking tot de validiteit van twee manieren om willingness to pay (WTP; bereidheid te betalen) te meten: de open vraag en een lijst met geldbedragen, de zogenoemde payment scale. We onderzochten de WTP voor een hoortoestel bij hoortoestelgebruikers, met zowel de open vraag als de payment scale. We hebben de resultaten van beide methoden vergeleken, en hebben de criteriumvaliditeit getest door beide te vergelijken met de eigen bijdrage die hoortoestelgebruikers daadwerkelijk voor hun hoortoestel betaald hadden. Constructvaliditeit werd getoetst door te onderzoeken of de WTP consistent is met de positieve inkomenselasticiteit. We vonden geen statistisch significant verschil tussen de resultaten van de open vraag en de payment scale. Beide methoden vertoonden goede criteriumvaliditeit, hoewel de open vraag een 
sterker verband liet zien met de daadwerkelijke eigen bijdrage. De open vraag had een betere constructvaliditeit, aangezien deze positief beïnvloed werd door inkomen. De resultaten van Hoofdstuk 4 wijzen erop dat de open vraag meer valide is dan de payment scale. Daarom bevelen we aan om in toekomstige WTP-studies naar hoortoestellen de open vraag te gebruiken om direct WTP-waarden te meten. Deze aanbeveling zou ook van toepassing kunnen zijn op andere studies waar respondenten bekend zijn met kosten of betalingen voor de interventie die geëvalueerd wordt.

Hoofdstuk 5 richtte zich op de ongelijkheid tussen willingness to accept (WTA; bereidheid te accepteren) en WTP in keuze-experimenten. Ons belangrijkste doel was het vergelijken van WTA en WTP in een keuze-experiment met betrekking tot de hoortoestelverstrekking. Daarnaast werden inkomenseffect en bezitsneiging (endowment effect) onderzocht als mogelijke verklaringen voor de ongelijkheid tussen WTA en WTP. Ook werd de impact van het gebruik van een WTA en/of WTP format om monetaire waarden te verkrijgen op de netto baten van ketenzorg in de hoortoestelverstrekking onderzocht. Mensen met klachten van slechthorendheid kregen willekeurig een WTA (kostenattribuut gedefinieerd als een korting op het hoortoestel) of een WTP (kostenattribuut gedefinieerd als extra kosten voor het hoortoestel) versie van het experiment. In de twee versies waren behalve het kostenattribuut alle keuzesets gelijk. De regressie-coëfficiënt van het kostenattribuut bleek statistisch significant hoger in de WTP versie dan in de WTA versie. Marginale WTA was statistisch significant hoger dan marginale WTP voor twee van de vier attributen. Het verschil tussen WTA en WTP was groter in de groep mensen met een hoger opleidingsniveau (als proxy voor inkomen). We vonden geen bewijs voor bezitsneiging in de vorm van een ervaringseffect. Het implementeren van ketenzorg in de hoortoestelverstrekking zou alleen aanbevolen worden wanneer gebruik gemaakt werd van de resultaten van het WTP format. Uit Hoofdstuk 5 blijkt duidelijk dat WTA hoger is dan WTP, ook in keuzeexperimenten. Aangezien dit de monetaire waarderingen beïnvloedt is er meer onderzoek nodig naar wanneer kortingen of extra kosten gebruikt moeten worden in het kostenattribuut, voordat de resultaten van keuze-experimenten gebruikt kunnen worden in kosten-baten analyses.

Hoofdstuk 6 onderzocht de voorkeuren van patiënten voor het overhevelen van aspecten van hoortoestelverstrekking van de medische sector (KNO-artsen en Audiologische Centra) naar audiciens, om inzicht te krijgen in de afwegingen van patiënten tussen verschillende aspecten van hoortoestelverstrekking. Respondenten hadden een voorkeur voor het eerste bezoek (de triage) bij de audicien, een hogere nauwkeurigheid bij het identificeren van mensen die medische zorg nodig hebben, een kortere duur van het totale proces van hoortoestelverstrekking en een eindcontrole bij de KNO-arts. Ze wilden een compensatie van minimaal $€ 17$ per twee maanden langere duur van het proces, $€ 54$ voor het eerste bezoek bij de KNO-arts, $€ 119$ per 10\% minder nauwkeurigheid en wilden een compensatie van $€ 227$ als ze de eindcontrole bij de KNO-arts zouden verliezen. Voorkeuren werden beïnvloed door geslacht, leeftijd, opleidingsniveau en ervaring met de hoortoestelverstrekking. Uit Hoofdstuk 6 blijkt dat slechthorenden open staan voor het overhevelen van aspecten van hoortoestelverstrekking van de medische sector naar audiciens. In de organisatie van hoortoestelverstrekking hebben slechthorenden een 
voorkeur voor een triage bij de audicien als deze minimaal 95\% nauwkeurig is, alsmede voor een eindcontrole bij de KNO-arts.

\section{Deel III}

Het derde deel van dit proefschrift integreert al het beschikbare bewijs in een beslismodel, om een afweging te maken tussen de voordelen en risico's van ketenzorg in de hoortoestelverstrekking.

Hoofdstuk 7 beschrijft de resultaten van het modelleren van de lange termijn kosteneffectiviteit van (verschillende vormen van) ketenzorg ten opzichte van de huidige organisatie van hoortoestelverstrekking, voor personen van 50 jaar en ouder. Hoortoestelverstrekking in de huidige vorm bleek iets effectiever en iets duurder dan ketenzorg, en had een grotere kans om kosteneffectief te zijn (54\%). Van de alternatieve vormen van ketenzorg had een zorgmodel met een triage bij de audicien en een eindcontrole bij de KNO-arts of het Audiologisch Centrum de grootste kans om kosteneffectief te zijn (40\%). Het is lonend om aanvullend onderzoek te doen om de onzekerheid omtrent deze resultaten te verminderen. Het is met name waardevol om te onderzoeken of mensen eerder hulp zoeken voor hun gehoorklachten als ze daarvoor direct bij de audicien terecht kunnen, en of de audicien in staat is om pathologie te detecteren.

In Hoofdstuk 8 worden de belangrijkste resultaten van dit proefschrift samengevat en bediscussieerd. Daarnaast worden enkele methodologische aspecten in beschouwing genomen. Eerst wordt de vraag gesteld of de Technology Assessment Iterative Loop direct bruikbaar is bij organisatievernieuwingen zoals ketenzorg in de hoortoestelverstrekking. Organisatievernieuwingen dienen namelijk geïmplementeerd te worden om ze te kunnen evalueren. Daarnaast bleek het besliskundig modelleren een bruikbaar instrument voor het evalueren van doelmatigheid in een zeer vroeg stadium, zelfs voordat overtuigend bewijs betreffende de werkzaamheid voorhanden is. Ten tweede zijn gerandomiseerde onderzoeken met controlegroep vaak niet toepasbaar bij organisatievernieuwingen. Pragmatische onderzoeken, die zowel voordelen als nadelen hebben ten opzichte van gerandomiseerde onderzoeken, zijn daarom vaak meer geschikt. Ten derde wordt in overweging gegeven dat aspecten van constructive technology assessment gebruikt zouden kunnen worden om het traditionele health technology assessment geschikt te maken voor organisatievernieuwingen. Deze aspecten zijn bijvoorbeeld het introduceren van technology assessment in een zeer vroeg stadium in de ontwikkeling van een technologie, en een continue dialoog tussen gebruikers van de technologie. Tenslotte wordt ingegaan op het feit dat modelleren altijd een simplificatie van de werkelijkheid is, en dat monetaire waarderingen zoveel meer kunnen bevatten dan de traditionele voor kwaliteit gecorrigeerde levensjaren, en daarom wel eens geschikter zouden kunnen zijn voor het evalueren van organisatievernieuwingen. 
Vervolgens worden een aantal aanbevelingen gedaan in Hoofdstuk 8. Naast de aanbevelingen die direct volgen uit de resultaten van de studies, wordt aanbevolen dat audiciens een gedragscode ontwikkelen. Ook wordt aanbevolen dat health technology assessment een meer prominente plek inneemt in het proces van organisatievernieuwing in de gezondheidszorg. Meer specifiek wordt aanbevolen dat bij organisatievernieuwingen in de gezondheidszorg health technology assessment al in een zeer vroeg stadium ingezet wordt.

Tenslotte worden aanbevelingen gedaan voor toekomstig onderzoek. Zo wordt aangegeven dat er consensus zou moeten komen over de uitkomstmaat in economische evaluaties in de gezondheidszorg: dienen voor kwaliteit gecorrigeerde levensjaren de aanbevolen uitkomstmaat te blijven, of is een bredere visie wellicht relevanter? Daarnaast is meer onderzoek nodig naar de validiteit van het direct meten van WTP. Meer onderzoek is ook nodig naar de vragen waarom het verschil tussen WTA en WTP zich voordoet in een keuze-experiment, en wanneer men kortingen of extra kosten dient te gebruiken in het kostenattribuut. Andere belangrijke gebieden voor verder onderzoek zijn de vragen of audiciens in staat zijn om patiënten die medische zorg nodig hebben te identificeren, en of ketenzorg in de hoortoestelverstrekking de drempel om hulp te zoeken voor gehoorklachten zal verlagen. 

Co-authors and affiliations 



\section{Lucien J.C. Anteunis, PhD}

Dept. of Otorhinolaryngology, Head \& Neck Surgery

University Hospital Maastricht, the Netherlands

Michelene N. Chenault

Dept. of Otorhinolaryngology, Head \& Neck Surgery

University Hospital Maastricht, the Netherlands

Dept. of Methodology \& Statistics

Maastricht University, the Netherlands

Adrian C. Davis, PhD

MRC Hearing \& Communication Group

University of Manchester, United Kingdom

Carmen D. Dirksen, PhD

Dept. of Clinical Epidemiology \& Medical Technology Assessment University Hospital Maastricht, the Netherlands

Wouter A. Dreschler, PhD

Dept. of Clinical and Experimental Audiology

Academic Medical Center Amsterdam, the Netherlands

Debby van Helvoort -Postulart, PhD

Dept. of Clinical Epidemiology \& Medical Technology Assessment University Hospital Maastricht, the Netherlands

Frans van der Horst, $\mathrm{PhD}$

Dept. of General Practice Maastricht University, the Netherlands

Manuela A. Joore, $\mathrm{PhD}$

Dept. of Clinical Epidemiology \& Medical Technology Assessment University Hospital Maastricht, the Netherlands

Alfons G.H. Kessels, MD, MSc

Dept. of Clinical Epidemiology \& Medical Technology Assessment University Hospital Maastricht, the Netherlands

Robert J. Stokroos, MD, PhD

Dept. of Otorhinolaryngology, Head \& Neck Surgery

University Hospital Maastricht, the Netherlands

Hans Verschuure, PhD

Audiological Center

Erasmus Medical Center Rotterdam, the Netherlands 

Dankwoord 

Dit proefschrift gaat over ketenzorg, over interdisciplinair werken. Het ultieme voorbeeld van een succesvol interdisciplinair samenwerkingsverband zijn mijn co-promotoren: Lucien Anteunis, Frans van der Horst en Manuela Joore. Ik besef me goed hoeveel geluk ik heb gehad dat ik werd begeleid door deze klinisch fysicus audioloog, medisch socioloog en HTA-onderzoeker. Bedankt dat jullie me deze kans hebben gegeven, voor alles wat ik van jullie heb geleerd en voor jullie vertrouwen in mij. Jullie zijn de belangrijkste reden dat ik dit proefschrift met zoveel plezier heb geschreven.

Manuela, omdat je op dezelfde afdeling zat als ik was jij meestal mijn eerste aanspreekpunt bij problemen of vragen. Jij hebt dus de meeste last van me gehad, maar daar staat tegenover dat ik geweldig veel van je heb geleerd. Bedankt dat ik altijd bij je kon binnenlopen. Je zit boordevol ideeën en ik weet dat ik nog heel veel van je kan leren, dus wat mij betreft blijven we nog lange tijd samenwerken! Lucien, je bent een inspirerend en motiverend, maar bovenal ook een heel prettig persoon. Bedankt dat je er voor me was, tijdens onze bijeenkomsten, als ik weer eens bij je kwam binnenvallen of in de auto na een vergadering. Vier jaar geleden hebben we na een erg gezellig etentje bij jou thuis afgesproken dat we dat na elk hoofdstuk van mijn boekje over zouden doen: de eerste is nu in ieder geval van mij! Frans, ik vond het erg prettig, maar ook heel bijzonder om met je samen te werken. Je humor, maar ook je kijk op het leven draagt daar voor een groot deel aan bij. Je bent sociaal heel sterk, wat belangrijk was voor een project als AZOS, maar ook voor mij persoonlijk.

Prof. dr. Manni, bedankt dat u mijn promotor wilde zijn, bedankt voor uw steun en met name voor uw grote vertrouwen in het team.

De leden van de beoordelingscommissie, te weten voorzitter prof. dr. B. Kremer, prof. dr. W.N.J. Groot, prof. dr. J.L. Severens, prof. dr. ir. A.F.M. Snik en dr. T. van der Weijden, wil ik van harte bedanken voor het beoordelen van dit proefschrift.

Mijn proefschrift had niet bestaan zonder al die bereidwillige en geduldige slechthorenden, audiciens, huisartsen, KNO-artsen en audiologen die vragenlijsten wilden invullen, geweldig bedankt daarvoor.

Aan de basis van dit proefschrift stond het AZOS-project. In het bijzonder wil ik daar Silvia Bours voor bedanken, die bergen werk heeft verzet voor het project en die er, naar mijn bescheiden mening, hoogstpersoonlijk voor heeft gezorgd dat Maastricht verreweg de meeste patiënten heeft geïncludeerd. Daarnaast bedank ik natuurlijk ook onze projectgroep, te weten Wout Dreschler, Hans Verschuure, Nienke Homans, Monique Boymans, Bas Franck, Arjo Rozeboom en Mariëlle Stokdijk. Ook wil ik de leden van het Nationaal Overleg Audiologische Hulpmiddelen, alsmede de afdeling KNO en het Audiologisch Centrum in Maastricht bedanken voor alles wat zij voor het project hebben gedaan. Het AZOS project was nooit wat geworden zonder de deelnemende audiciens, in het bijzonder Jos Bosch, Carlo Habets en Peter Reiters. Ontzettend bedankt voor jullie tomeloze inzet, maar zeker ook voor jullie enthousiasme en gezelligheid. 
Twee hoofdstukken van dit proefschrift zijn het resultaat van een ander project dat we hebben gedaan. Jolien en Judy, hartstikke bedankt voor al het werk dat jullie hebben verzet en het geduld dat jullie hebben gehad. Ook wil ik iedereen van Hans Anders Maastricht, Beter Horen Maastricht en Streukens Heerlen en Maastricht bedanken voor hun medewerking.

De artikelen in dit proefschrift heb ik uiteraard niet alleen geschreven. Ik ben dan ook erg dankbaar voor de hulp van alle co-auteurs. Fons, Carmen en Debby, bedankt voor jullie hulp bij het discrete choice experiment. Fons, om maar even wat psychologen-statistiek te gebruiken: zonder jou waren hoofdstuk 5 en 6 statistisch significant minder valide (en leuk om te schrijven) geweest ( $p$-value 0.001). Mickey, ook jij bedankt voor je hulp bij de statistiek, en bij de Engelse taal. Maar ook voor je interesse, hoe druk je het zelf ook had. Professor Davis, Adrian, thanks for your help on chapter 6. Hopefully this is the start of a fruitful Maastricht-Manchester cooperation! Robert Stokroos, bedankt voor je hulp bij hoofdstuk 7. Jan Duijvestijn, bedankt dat ik je data mocht gebruiken voor het laatste artikel en veel succes met jouw eigen boekje. Harry Streukens, bedankt voor je enthousiasme en je hulp bij de studies in dit proefschrift.

Uiteraard wil ik al mijn collega's bedanken. Zowel bij KEMTA als bij het AC en de MTAclub had ik collega's die altijd bereid waren me te helpen en het werk vele malen leuker maakten. Speciaal wil ik daarvoor Daniëlle bedanken, voor alles, maar ook Irene, Ester en Edith, die in de afgelopen jaren zo ontzettend veel voor mij geregeld hebben.

Anita en Susanne, super bedankt dat jullie er voor me zijn, niet alleen nu als paranimf, maar altijd. Bianca, bedankt voor je hulp bij het ontwerpen van de kaft van mijn boekje.

Tenslotte denk ik dat een lieve, grappige, relativerende, geïnteresseerde en begripvolle superheld (met een drukke baan), collega's die in hetzelfde schuitje zitten, collega's om mee te squashen, wandelen of lunchen, een fantastische en flexibele baas, maar ook lieve, geïnteresseerde en voor de nodige ontspanning zorgende ouders, broers, schoonouders, ooms (THX MCHL!), nichies, vrienden en familie essentieel zijn om het afronden van je proefschrift in combinatie met een nieuwe baan te overleven. Dankzij jullie heb ik het gered, en hopelijk hebben jullie genoten van de rust, want vanaf nu heb ik eindelijk weer tijd voor jullie! 
Curriculum Vitae 

Janneke Grutters was born on March 30, 1980, in Rijkevoort, the Netherlands. She attended primary school at De Klimop in Rijkevoort and secondary school at the Elzendaal College in Boxmeer. After graduation she studied Health Sciences at the University of Maastricht, with specialization Work \& Health, which she finished in 2002.

In 2003 she started as a research assistant at the University Hospital Maastricht, with the departments of Otolaryngology, Head \& Neck Surgery and Clinical Epidemiology \& Medical Technology Assessment. Here she worked on a project regarding the social impact of not reimbursing hearing aids. Later that year she started a new project at the same departments. In this AZOS (Aangepast ZOrgmodel Slechthorendheid) project she was involved as researcher. The AZOS project was a collaboration between the University Hospital Maastricht, the Amsterdam Medical Centre and the Erasmus Medical Centre in Rotterdam. After finishing the project in 2006 she has performed additional research regarding the organization of hearing aid provision. Since May 2007 Janneke works as a post doc researcher at MAASTRO clinic in Maastricht, where she evaluates the costeffectiveness of radiotherapy with particles (protons and carbon-ions) in the treatment of lung cancer. 

Publications 



\section{Papers}

Grutters JPC, van der Horst F, Joore MA, Verschuure J, Dreschler WA, Anteunis LJC. Potential barriers and facilitators for implementation of an integrated care pathway for hearing-impaired persons: an exploratory survey among patients and professionals. BMC Health Services Research 2007, 7:57.

Grutters JPC, Joore MA, Van der Horst F, Verschuure J, Dreschler WA, Anteunis LJC. Choosing between measures: comparison of EQ-5D, HUI2 and HUI3 in persons with hearing complaints. Quality of Life Research 2007, 16 (8): 1439-1449.

Grutters JPC, Anteunis LJC, Chenault MN, Joore MA. Willingness to Pay for a hearing aid: comparing the payment scale and open-ended question. Journal of Evaluation in Clinical Practice, accepted for publication.

Grutters JPC, Kessels A, Dirksen C, Van Helvoort-Postulart D, Anteunis LJC, Joore MA. Willingness to Accept versus Willingness to Pay in a Discrete Choice Experiment. Submitted.

Grutters JPC, Joore MA, Kessels A, Davis AC, Anteunis LJC. Patient preferences for direct hearing aid provision by a private dispenser. A discrete choice experiment. Ear and Hearing, accepted for publication.

Grutters JPC, Joore MA, Van der Horst F, Stokroos RJ, Anteunis LJC. Decision-analytic modeling to assist decision-making in organizational innovation: the case of shared care in hearing aid provision. Submitted.

Pijls-Johannesma M, Grutters JPC, Lambin P, De Ruysscher D. Particle therapy in lung cancer: where do we stand? Cancer Treatment Reviews, accepted for publication.

\section{Reports}

Joore MA, Grutters JPC, Chenault MN, Anteunis LJC. Maatschappelijke impact van het niet vergoeden van hoortoestellen. 2003, Nationale Hoorstichting.

AZOS werkgroep. Eindrapport AZOS. 2006, College voor Zorgverzekeringen. 


\section{Presentations}

Grutters JPC, Joore MA, Dreschler WA, Verschuure J, Anteunis LJC. The care for the hearing-impaired before deregulation. Dutch Society of Audiology, January 28 2005, Utrecht, the Netherlands. Invited oral presentation.

Grutters JPC, Joore MA, Dreschler WA, Verschuure J, Anteunis LJC. Towards direct hearing aid provision in the Netherlands: facilitators and barriers. European Federation of Audiological Societies Congress, June 19-22 2005, Göteborg, Sweden. Oral presentation.

Grutters JPC, Joore MA, Verschuure J, Dreschler WA, Anteunis LJC. Comparison of EuroQol and Health Utilities Index Mark III before and after hearing aid fitting. World Congress of the International Health Economics Association, July 10-13 2005, Barcelona, Spain. Poster presentation.

Grutters JPC, Joore MA, Van der Horst F, Anteunis LJC. Economic evaluation of direct hearing aid provision. Netherlands Society of Otorhinolaryngology and Cervico-Facial Surgery and Dutch Society of Audiology. April 6, 2006, Nieuwegein, the Netherlands. Invited oral presentation.

Grutters JPC, Joore MA, Kessels A, Davis AC, Anteunis LJC. Patient preferences for direct hearing aid provision by a private dispenser. A discrete choice experiment. European Federation of Audiological Societies Congress, June 6-9 2007, Heidelberg, Germany. Oral presentation.

Grutters JPC, Kessels A, Dirksen C, Van Helvoort-Postulart D, Anteunis LJC, Joore MA. Willingness to Accept versus Willingness to Pay in a Discrete Choice Experiment. World Congress of the International Health Economics Association, July 8-11 2007, Copenhagen, Denmark. Oral presentation. 

But if the world could remain

within a frame

like a painting on a wall.

Then I think we would see the beauty.

Then we would stand staring in awe

at our still lives posed

like a bowl of oranges

like a story told

by the fault lines and the soil.

Bright Eyes, Bowl of Oranges (Lifted, or the story is in the soil, keep your ear to the ground) 